\title{
Population changes in rattail species on the Chatham Rise
}

by

Kathleen Large

\author{
A thesis \\ submitted to the Victoria University of Wellington \\ in fulfilment of the \\ requirements for the degree of \\ Master of Science \\ in Statistics.
}

Victoria University of Wellington

2014 



\begin{abstract}
The aim of this project was to conduct a stock assessment to determine the population dynamic characteristics of rattail species taken as bycatch in the hoki, hake and ling fishery on the Chatham Rise. No quantitative assessment of the current size of rattail populations, and how these may have changed over time, has been carried out before. There is interest in the need to quantify the impact of commercial fishing on the rattail populations, as rattails (Macrouridae family) are considered to be an ecologically important species complex in the deep ocean, and there may be the potential for the development of a commercial fishery based on their value as processed fishmeal. The minimum data required for a stock assessment are an abundance index and a catch history. Abundance indices are available for over 20 species of rattail produced from scientific surveys conducted annually on the Chatham Rise since 1992. Catch histories for individual rattail species in the same area are not available. A method was developed to reconstruct commercial catches of rattails from commercial effort data and survey catch and effort data. A surplus production model was fitted to the reconstructed catch data and survey abundance indices, using maximum likelihood and Bayesian methods to estimate model parameters and uncertainty. A surplus production model has two components: an observation model for abundance indices and a process model for population dynamics. Maximum likelihood estimation was applied to a model that specified errors for the observations only, and this produced estimates that had wide confidence intervals. A Bayesian approach was then taken to fit a statespace version of the model that incorporates errors associated with the observation and process models. While the Bayesian method produced more plausible parameter estimates (in comparison to the maximum likelihood method) and parameter uncertainty was reduced, our analysis indicated the posterior estimates were highly sensitive to the specification of different priors. There may be several reasons for these results, including: the small number of observations, lack of contrast in the data and mis-specification of the model. Meaningful estimates of the absolute size of rattail populations are not possible with these results, where estimates can vary by orders of magnitude depending on prior specification. This implies that more work needs to be done to develop more effective methods that can be used to help inform decisions regarding the management of these fish populations. Improving data collection, investigating informative priors and extending/respecifying the model are considered worthwhile avenues of future work to improve stock assessments of rattails.
\end{abstract}




\section{Acknowledgments}

I would like to thank MPI (Ministry for Primary Industries) and NIWA (National Institute of Water and Atmospheric Research) for their support of my post-graduate study through the provision of a post-graduate scholarship in Quantitative Fisheries Science. Special thanks to Nokuthaba Sibanda, Richard Arnold and Matt Dunn for their guidance and encouragement. Thanks to Darcy for generously sharing his knowledge. And, thanks to Boyd, Thanh, Xiaoyu, Thuong, Roy, Daniel, Craig and Kimberley for their support and generosity. 


\section{Contents}

1 Introduction 1

1.1 Research question . . . . . . . . . . . . . . . . . . 1

1.2 Research approach and objectives . . . . . . . . . . . 2

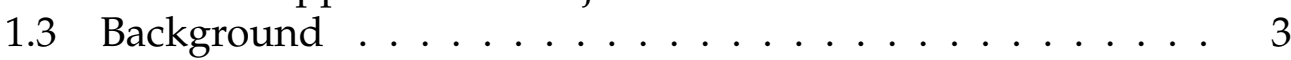

2 Data 9

2.1 Abundance indices ......................... 9

2.1.1 Biomass estimates by species . . . . . . . . . . . 11

2.2 Commercial catches . . . . . . . . . . . . . . . . . 13

2.2.1 Reconstructing catch histories . . . . . . . . . 13

3 Stock assessment methods 25

3.1 Surplus production model . . . . . . . . . . . . . . 28

4 Maximum likelihood estimation of observation model parameters $\quad 35$

4.1 Maximum likelihood estimation . . . . . . . . . . . 35

4.2 Estimation procedure . . . . . . . . . . . . . . 37

4.3 Model assessment . . . . . . . . . . . . . . . . . 39

4.4 Results ...................... . . 41

4.4 .1 Namibian hake data . . . . . . . . . . . . . 41

4.4 .2 Rattail data . . . . . . . . . . . . . . 44

5 Bayesian estimation of state-space model parameters 47

5.1 Bayesian estimation . . . . . . . . . . . . . . . . . . 48

5.2 State-space surplus production model . . . . . . . . . . . . 49

5.3 Specification of prior distributions . . . . . . . . . . 51

5.4 Sampling from the posterior distribution . . . . . . . . 53

5.5 Results ....................... 59

6 Discussion $\quad 65$

$\begin{array}{lr}\text { References } & 69\end{array}$ 
vi

$\begin{array}{ll}\text { Appendices } & 79\end{array}$

Appendix A: Rattail codes and description summary . . . . . . 81

Appendix B: Rattail codes and survey summary . . . . . . . . . . 82

Appendix C: Summary of bycatch analysis . . . . . . . . . . . 83

Appendix D: Map, core survey strata . . . . . . . . . . . . . . . . 89

Appendix E: List, core survey strata polygons . . . . . . . . . . . . 90

Appendix F: Table, commercial effort by stratum and year . . . . 93

Appendix G: Catch and index data series, 1992 - 2012 . . . . . . . 94

Appendix H: MLE results for CBO, COL and CAS . . . . . . . . . 95

Appendix I: WinBUGS code for state-space production model . . 98

Appendix J: Bayesian estimation results . . . . . . . . . . . . 99

Appendix K: Posterior estimates for CBO, COL and CAS . . . . . 102 


\title{
List of abbreviations
}

\author{
AIC Akaike Information Criteria \\ CPUE Catch Per Unit Effort \\ CR Chatham Rise \\ EEZ Economic Exclusion Zone \\ MLE Maximum Likelihood Estimate \\ MPI Ministry for Primary Industries \\ MSY Maximum Sustainable Yield \\ NIWA National Institute of Water and Atmospheric Research \\ QMS Quota Management System \\ SOP Scientific Observer Programme \\ TACC Total Allowable Commercial Catch
}

\section{Common fisheries science modeling notation}

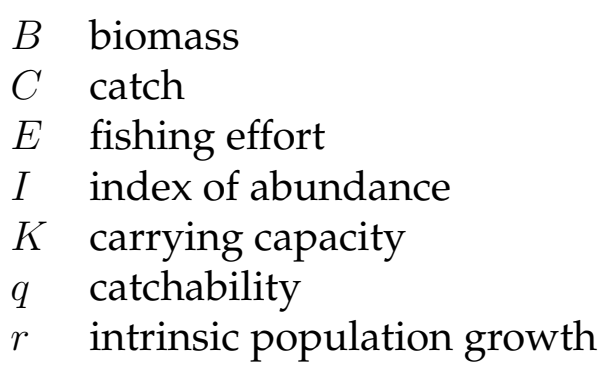

\section{Note on fishing year}

The fishing year extends from 01 October to 30 Sepetember. We assign observations and estimates for this time period to the year containing the latest set of dates. For example, $C_{t}$ where $t=2010$, denotes catches from 01 October 2009 - 30 September 2010. 


\section{Chapter 1}

\section{Introduction}

This research uses administrative and survey data from Ministry for Primary Industries (MPI) databases to determine changes in the population size of rattail fishes in response to fishing pressure in New Zealand's deep sea trawl fisheries over the last 20 years. This research will help assess the size and status of these populations; and therefore the risk from fishing they may face.

The results will be useful to policy makers in MPI in developing management strategies for rattails that have the potential to be targeted as a commercial fishery and/or as part of a broader ecosystem management regime.

\subsection{Research question}

How do we estimate population dynamics of multiple species of rattails, commonly caught as bycatch in New Zealand fisheries, with a view to modelling population changes that can help inform fisheries management of rattails as a species group? 


\subsection{Research approach and objectives}

This project will focus on rattail populations associated with the Chatham Rise (CR) hoki fishery. The hoki fishery is New Zealand's largest deepsea fishery and is managed under one TACC (total allowable commercial catch) and assessed as two stocks, western and eastern [67]. Rattails are a major bycatch of the fishery targeting hoki on the Chatham Rise (the eastern stock), where the fishery operates all year round. The western stock is based on the South Island's hoki spawning grounds, where hoki are particularly concentrated for a limited season and can be targeted as a singlespecies directed-fishery. As such, bycatch rates from the hoki fishery on the western stock are comparatively low. Also, importantly, annual scientific research trawl surveys have been conducted on the Chatham Rise since 1992, providing at the same time a fisheries-independent abundance index for rattails, by species. Such quality data are not available for any other fishing area in New Zealand's Economic Exclusion Zone (EEZ).

We make the assumption here that the rattails on the Chatham Rise belong to one stock, all residing in this one area (i.e. continuous presence, no immigration or emigration) and all species of rattails are equally vulnerable to the hoki fishery.

We will fit surplus production models (defined below) to commercial catch data to estimate population dynamics in terms of abundance indices. An index of abundance is a relative (i.e. non-absolute) measure of the size of a fishery stock, and it can be used to compare changes over time and space; a multiplicative constant is used to scale the index to an absolute size, e.g. biomass (tonnes, say) of fish.

We investigae Maximum Likelihood and Bayesian approaches for esimation. Estimated model parameters from the fitting process can then be used in a simulation to project the population biomass by species (driven by rattail catch trends associated with different management strategies). 


\subsection{Background}

Rattails (family Macrouridae) are a ubiquitous and abundant family of deep sea fishes that live on or near the ocean floor. Widely distributed, rattails occur in all oceans, predominantly at depths between 200 and $2000 \mathrm{~m}$, but some also at abyssal depths to $6000 \mathrm{~m}$ [49]. Also known as grenadiers, nearly 400 species are identified worldwide [50] with over 60 species in New Zealand's EEZ and 41 species known from the Chatham Rise [88] (Figure 1).

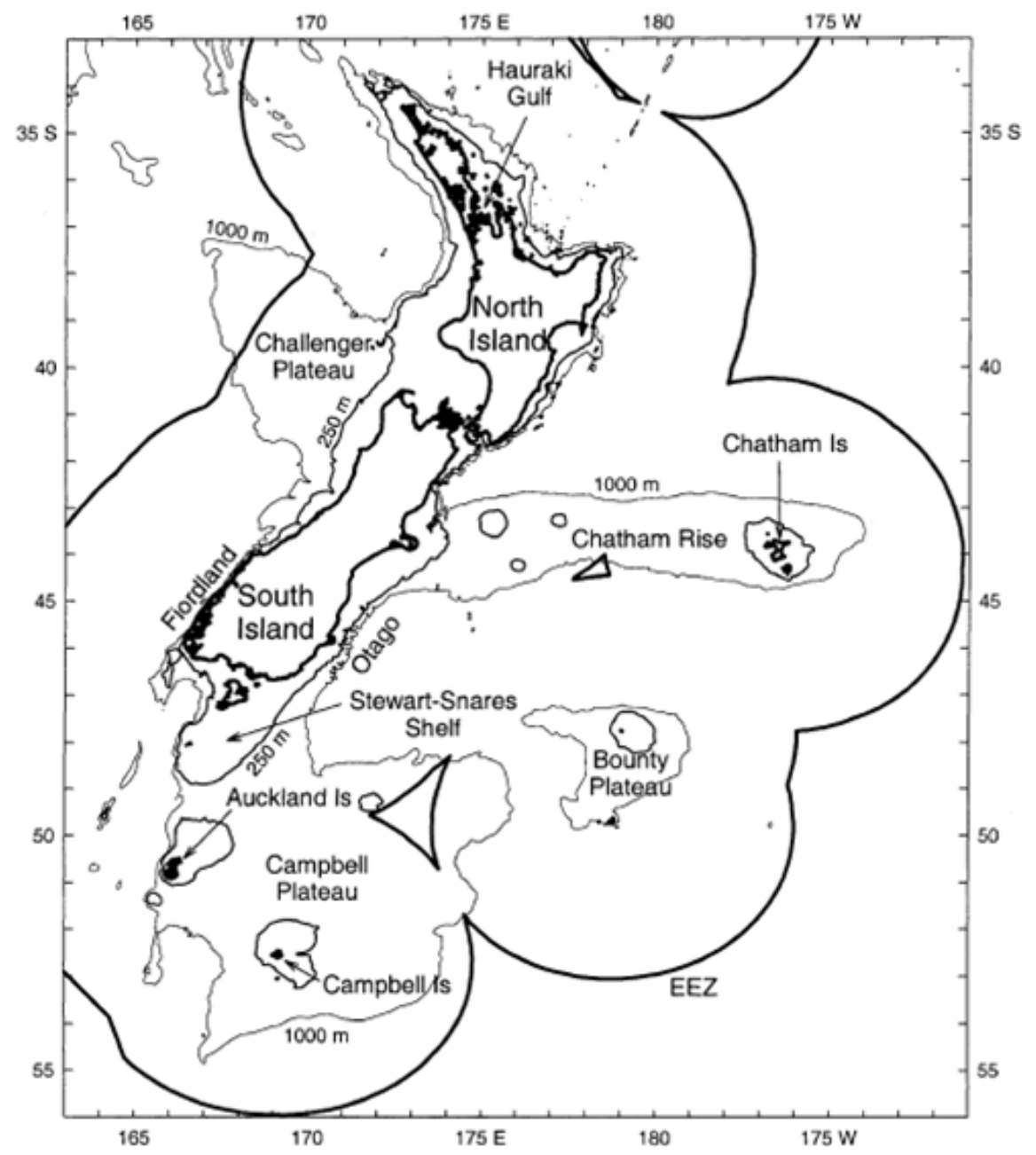

Figure 1: New Zealand region showing the Economic Exclusion Zone (EEZ) and geographic and bathymetric features, including the Chatham Rise. Illustration from Francis, Hurst, McArdle, Bagley and Anderson (2002) [30]. 
Rattails are among the most abundant demersal (living on or near the sea floor) fishes caught in trawls at depths greater than 200m [72] and among the most frequently caught species on New Zealand's continental slope [89]. They are estimated to account for $10 \%$ of fish biomass on the upper continental slope (200-800m) of the CR [88], and densities of rattails in the Kaikoura Canyon (bordering west CR) are estimated to be of a magnitude higher than total fish abundance for bathyal slopes in the Northeast Atlantic [22]. Despite this abundance, rattail biology in New Zealand remains largely unknown [87].

Rattails are caught in significant numbers as incidental bycatch in nearly every deep-sea fishery [20] world-wide, including most of New Zealand's deep-sea, demersal trawl fisheries [13]. Thirty-nine species are described in the New Zealand field guide to common species caught in bottom and mid-water trawling [71] (summary in Appendix A) which highlights the varying depth ranges and sizes of the different species, with the biology and ecology of most species described as largely unknown. During bottom trawling a net (trawl), attached by cables to a fishing vessel, is dragged along the sea floor (Figure 2). The body of the trawl is like a funnel narrowing towards a codend, the trailing end of the net where the fish are caught (Figure 3). Floats attached to the cable that runs along the upper mouth of the net (headline) hold the net open and weighted bobbins are attached to the cable that runs along the lower mouth of the net (footrope). Large metal doors (otter boards or trawl doors) are also used to keep the mouth of the trawl open, and are positioned in such a way that the hydrodynamic forces acting on them when the net is towed pushes them outwards [92].

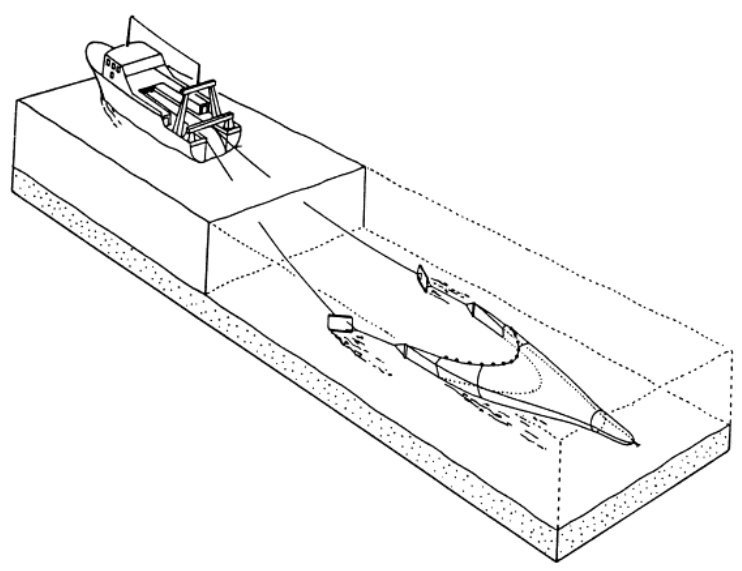

Figure 2: Generic bottom trawling setup of vessel towing deployed trawl gear. Illustration from Manche Maree website [92]. 

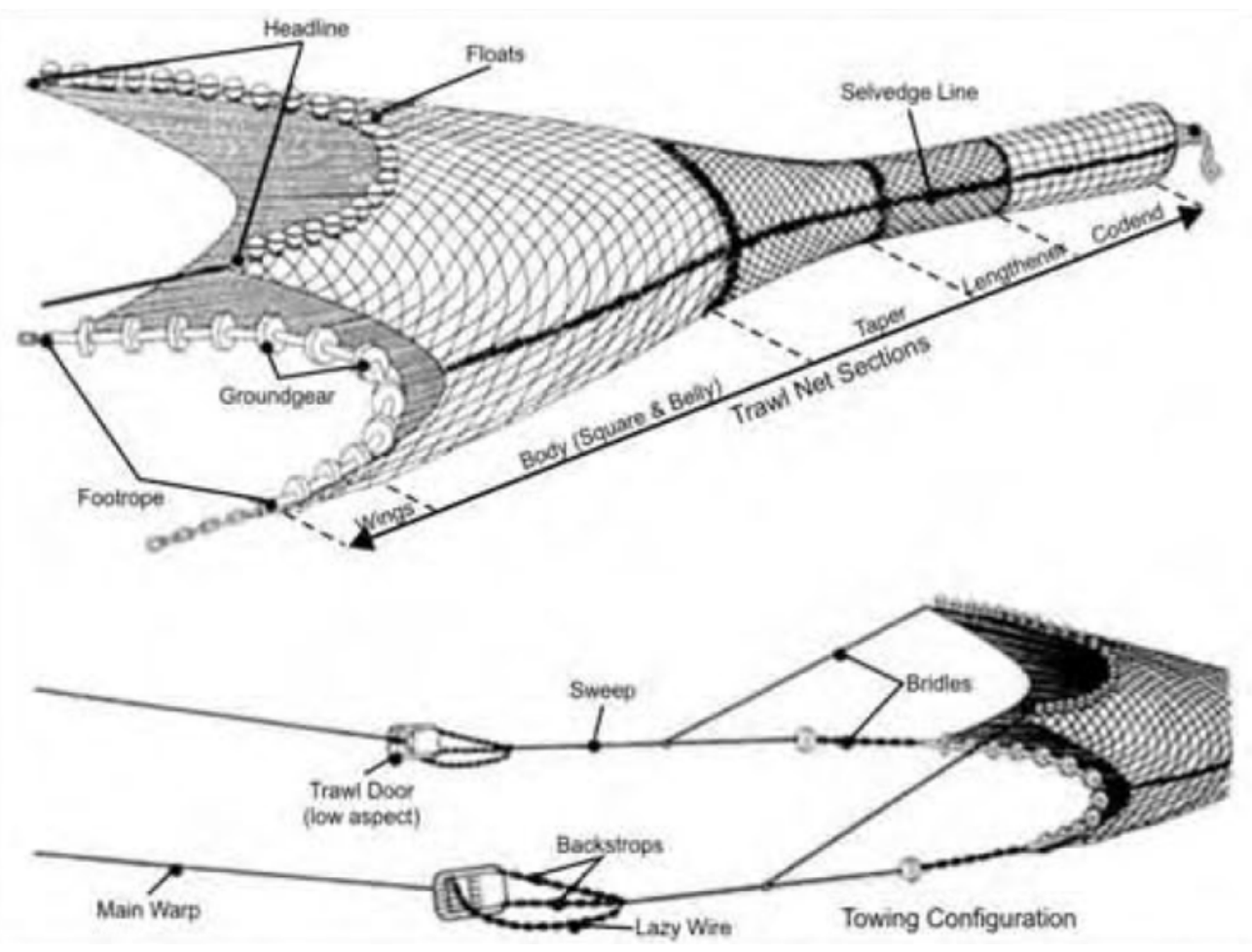

Figure 3: Configuration of a typical bottom trawl net during trawling. Illustration from New Zealand Ministry of Fisheries Bottom Fishery Impact Assessment Report (2008) [69].

Along with spatial and temporal factors (i.e. where and when), several other factors determine the final makeup of the catch, such as trawl gear set-up (including the mesh-size of the net in the codend) and deployment, the vulnerability of the different species in the path of the trawl to being caught, and the skill of vessel crew in targeting particular species. What is hauled on-board will be some mixture of intended target species and other non-target species (bycatch), where some of the bycatch species may also be of commercial value.

The current method of monitoring the impact of fishing effort on rattail populations is by way of reporting the annual observed rattail bycatch from commercial trawls surveyed in the MPI scientific observer programme (SOP). As it can be difficult for non-specialists to differentiate between species of rattails, observers usually record the generic code "RAT" for most species and "JAV" code for the particularly abundant rattail species, javelinfish (Lepidorhynchus denticulatus). For example, the amount of rattails identified as bycatch in the Chatham Rise hoki fishery has, most recently, been above $10 \%$ of the hoki catch (Figure 4) and is higher than the bycatch total of identified major commercial species (e.g. hake, ling, silver 
warehou).

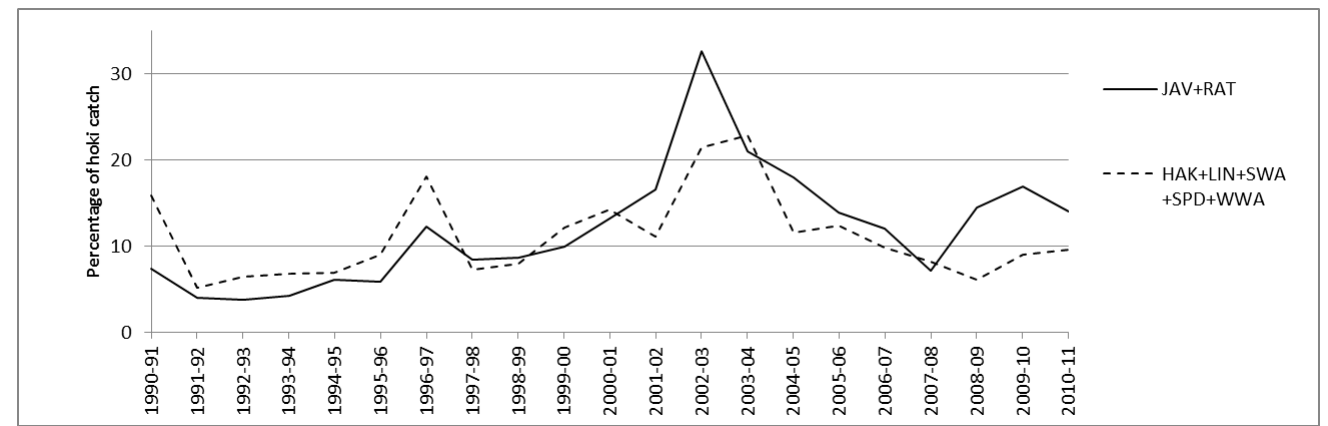

Figure 4: Bycatch weight as a percentage of hoki catch weight on vessels with SOP observers in the Chatham Rise hoki fishery, for tows targeting hoki, from fishing years 199091 to 2010-11. RAT (Rattails) and JAV (Javalinfish) codes combined to give Macrouridae catch as \% of hoki catch. HAK (Hake), LIN (Ling), SWA (Silver warehou), SPD (Spiny dogfish) and WWA (White warehou) codes combined to give catch of main QMS-species as \% of hoki catch. Percentage bycatch extracted from Table 6e in Ballara and O'Driscoll (2012) [12].

Periodically, these SOP data observations are used to estimate the total bycatch of rattails and javelinfish in commercial fisheries (as summarised in Appendix B). Also, scientific trawl surveys, conducted annually on the Chatham Rise since 1992 (where accurate differentiation between species is achieved), give an indication of the relative abundance of individual rattail species from year to year. However, quantitative assessment of the current size of rattail populations, and how these may have changed over time, has not been carried out.

New Zealand fisheries policy specifies that the impact of a fishery on bycatch species be monitored [70], and the standard method of measuring this impact is by way of a stock assessment. Stock assessments combine various data, for example the results of scientific research, catch reports from commercial fisheries and data from the observer programme, to produce assessments of the status of a fish stock. For New Zealand's EEZ, the Ministry for Primary Industries (MPI) currently evaluates 348 stocks of the 636 in its Quota Management System (QMS). Those not evaluated are considered to be nominal stocks, "for which a significant commercial or non-commercial potential has not been demonstrated" [68]. No rattail species are currently included in the QMS.

There is increasing interest in the need to quantify the impact of commercial fishing on rattail populations because: (1) by virtue of their abundance they are now considered to be an ecologically important species complex 
in the deep ocean [24,87]; and (2) their potential as a commercially targeted species may become a reality, driven by continued pressure on fishers to lower discard rates and increasing economic returns for processing previously discarded bycatch into fishmeal [6].

To attempt stock assessments for individual species of rattails, commercial catch data by species is required. No such data are currently collected, as commercial fishing operations are not required to report catch information for non-QMS species. Only SOP data is available for rattails and this is not recorded at species level. Also, if rattails were to be considered for management within the QMS, it seems unlikely (given the difficulties in telling species apart) that they would be managed at species level, as fishing effort would be targeted at rattails as a group. Therefore, catch data (by way of the observer programme) would continue to be collected at the aggregated level, i.e. data collection systems would not be changed to collect species specific catch data for rattails.

However, it would be naive and of limited value to fisheries managers to provide a stock assessment of rattails as a single stock. The depth range of rattail species caught in the Chatham Rise hoki fishery varies (Appendix B), and for some species extends beyond the fishing trawl depth. In addition, research indicates that the biology and life history of different rattail species can vary [87], e.g. some may be shorter lived and more productive, others longer lived and less productive. Therefore, fishing pressure may have different effects on different rattail species. Consequently, management strategies that work well for one or some species, may not work well for all. It may, in fact, not be possible for fisheries managers to develop strategies that will work well for all rattail species. However, it is beyond the scope of this project to determine how these strategies might be developed.

The objective of this project is to provide information based on current data collection systems to inform the decision making process for rattail stock management. The problem is to determine how to use speciesaggregated catch histories in single-species stock assessments to determine a multiple-species response to fishing pressure.

Chapter 2 proposes a method to construct commercial catches of rattails from commercial effort data and survey catch and effort data; Chapter 3 outlines stock assessment methods and introduces the surplus productiom model; Chapter 4 presents the maximum likelihood estimation method and estimation results for the surplus production (observation) model; 
Chapter 5 presents the Bayesian estimation of a state-space surplus production model parameters; and Chapter 6 includes a discussion of the results and outlines possible future work indicated by this study. 


\section{Chapter 2}

\section{Data}

Data available for this project were abundance indices by rattail species from fisheries-independent scientific surveys and commercial fishing effort records collected for administrative purposes.

\subsection{Abundance indices}

These data are collected from an annual series of surveys contracted by MPI and conducted by NIWA. Data have been provided as summarised biomass statistics extracted and made available by NIWA from the Empress database for 17 rattail species in 21 summer surveys conducted from 1992 to 2012.

These statistics were summarised as abundance indices and are calculated from data collected on the Chatham Rise trawl surveys, conducted annually since 1992 by NIWA from the research vessel Tangaroa. An abundance index is made up of a series of area weighted catch estimates, assumed to be proportional to population biomass. The main aim of the survey is to provide relative biomass estimates of adult and juvenile hoki. Many other species are also monitored (including rattails), and for most of these species the trawl survey is the only fisheries-independent estimate of abundance on the Chatham Rise [71].

Survey catch and effort are spatially stratified to optimize the estimation of relative biomass indices of the main target species in the area [34]. Primarily this is hoki, but the survey is also optimized for hake and orange roughy in deepwater strata (added later in the series). In the survey, the metric of effort is based on samples of swept area, with swept area defined as the width $(\mathrm{m})$ of the net doors multiplied by the length $(\mathrm{km})$ of a survey 
trawl. Assuming a constant net height (the vertical opening of the net), this gives the total area of fishing effort that fish are vulnerable to, during the time that the net is deployed along the sea floor. It is assumed that fish are randomly distributed within a catch stratum, that no fish are present above the height of the headline, and that all fish within the path of the trawl doors are caught [71]. Therefore, the catchability coefficient (an estimate of the proportion of fish in the path of the net which are caught), determined by the product of vulnerability, vertical availability, and areal availability, is assumed to be 1 [48].

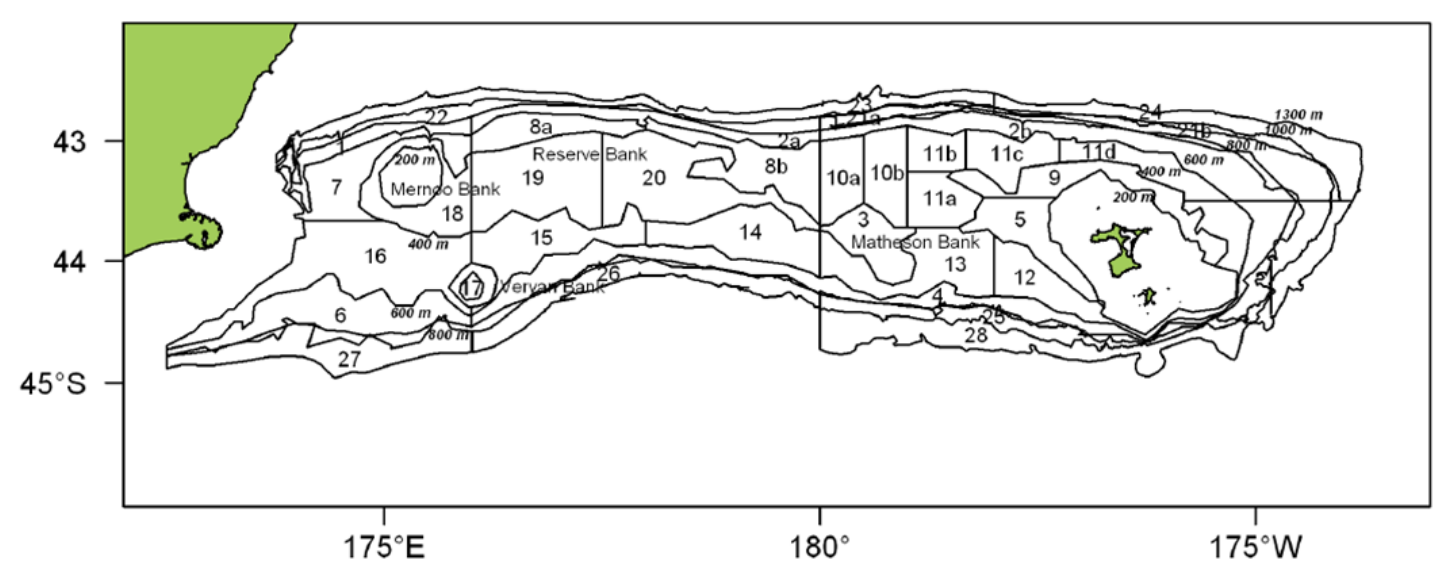

Figure 5: Chatham Rise trawl survey (January 2011) showing stratum boundaries. Excerpt from Stevens, O’Driscoll, Dunn, Ballara and Horn (2012) [89].

Catch per unit area varies depending on location within the Chatham Rise. To reduce variability, the area is stratified, and random samples are taken within each stratum. The survey is a two phase stratified random design (as described by Francis [32]), in which the allocation of each sampling location (trawl station) to strata in phase 2 is based on biomass estimates obtained in phase 1 . The number of samples (one trawl per station location) within each stratum is assigned via an algorithm where the phase 1 allocation is based on biomass estimates from previous years. Additional allocations are made in phase 2 , based on biomass estimates from phase 1. Stratification (Figure 5) uses 200m depth intervals (200-400m, 400-600m and $600-800 \mathrm{~m}$ ), longitude and latitude across the Chatham Rise. Phase 1 station positions are selected randomly before the voyage, and at each station the trawl is towed for 3 nautical miles at a speed over the ground of 3.5 knots.

The standardisation of sample collection, analysis and reporting of the sur- 
veys conducted by the R.V. Tangaroa is described in Hurst et. al. (1993) [48]. The development of such standardisation procedures is considered essential to ensure continuity between years and within voyages. Procedures cover aspects of trawl deployment such as setting and monitoring the optimum door-spread during the trawl, monitoring and maintaining constant bottom-trawl depth and tow speed, and recommendations for when a trawl encounters foul ground or when lack of daylight limits allocated trawling time. At each station all items in the catch are sorted into species and weighed, and length frequency and biological sampling is carried out, with procedures also detailed for these tasks. Survey reports are published by NIWA describing the survey objectives, design, sampling and data analysis, e.g. Trawl survey of hoki and middle-depth species on the Chatham Rise, January 2011 (TAN1101) [89].

\subsubsection{Biomass estimates by species}

For each species, biomass estimates in the survey area are based on doorspread using the swept area method [31,33]. The station areal density $\left(\mathrm{kg} / \mathrm{km}^{2}\right)$ is calculated from the catch weight of the species and the swept area at each sampling station. The stratum mean density is calculated from the areal density of the stations within the stratum. The stratum biomass is the stratum mean density multiplied by the stratum area. Finally, the species biomass estimate for the survey is the sum of the species biomass from all strata.

Species biomass estimation is implemented by custom software SurvCalc [19] (a C++ computer program developed in 2008 to analyse data from stratified random surveys), where

- $C_{i}$ is the catch weight $(\mathrm{kg})$ at station $i$,

- $f_{i}$ is the effective fished area $\left(\mathrm{m}^{2}\right)$ in station $i$,

- $n_{s}$ is the number of selected station in stratum $s$,

- $a_{s}^{\prime}$ is the area $\left(\mathrm{km}^{2}\right)$ of stratum $s$,

- $i$ indexes the selected stations for the survey, and

- $s$ indexes the selected strata for the survey;

and with

- station areal density $\left(\mathrm{kg} / \mathrm{km}^{2}\right)$

$$
A D_{i}=10^{6} C_{i} / f_{i}
$$


- stratum mean areal density $\left(\mathrm{kg} / \mathrm{km}^{2}\right)$ and standard error

$$
A D_{s}=\frac{1}{n_{s}} \sum_{i=1}^{n_{s}} A D_{i}, \quad \operatorname{se}\left(A D_{s}\right)=\sqrt{\frac{\sum_{i=1}^{n_{s}}\left(A D_{i}-A D_{s}\right)^{2}}{n_{s}\left(n_{s}-1\right)}}
$$

- stratum biomass $(\mathrm{kg})$ and standard error

$$
B_{s}=A D_{s} a_{s}^{\prime}, \quad \operatorname{se}\left(B_{s}\right)=\operatorname{se}\left(A D_{s}\right) a_{s}^{\prime}
$$

- survey area biomass (tonnes), standard error and coefficient of variation

$$
B=\sum_{s} B_{s}, \quad \operatorname{se}(B)=\sqrt{\sum_{s} \operatorname{se}\left(B_{s}\right)^{2}}, \quad \operatorname{cv}(B)=\operatorname{se}(B) / B
$$

NIWA extracted and provided data for 17 rattail species from 21 trawl surveys (1992 to 2012) as:

$B_{s j t} \quad$ biomass $(\mathrm{kg})$ for year $t$, for species $j$, in stratum $s, \operatorname{with} \operatorname{cv}\left(B_{s j t}\right)$

$B_{j t} \quad$ biomass $(\mathrm{kg})$ for species $j$ in year $t$, with $\mathrm{cv}\left(B_{j t}\right)$

In a review of hoki and middle-depth trawl surveys of the Chatham rise (January 1992-2010) [71], part of the study described how well biomass was estimated for sampled species (based on the estimated biomass c.v.'s), with the following classifications:

"Biomass of this species is very well / well / moderately well / poorly estimated in the core survey area.

- Very well = mean c.v. $<20 \%$

- Well = mean c.v. 20 to $30 \%$

- Moderately well $=$ mean c.v. 30 to $40 \%$

- Poorly = mean c.v. $>40 \% "$

The study also described the appropriateness of the core survey area and depth for a species, i.e. "approriate if the species distribution is usually between 200 and $800 \mathrm{~m}$ and not appropriate if the distribution is typically deeper or shallower, or the species is known to occur mainly in midwater". The survey area approriateness and biomass estimate quality reported for surveyed rattail species is summarised in Appendix B. 
For four surveyed rattail species, Coelorinchus aspercephalus (Oblique banded rattail), Coelorinchus bollonsi (Bollon's rattail), Coelorinchus oliverianus (Oliver's rattail), and Lepidorhynchus denticulatus (Javelinfish), the core survey area and depth range is described as appropriate and the biomass estimate is classified as very well, moderately well or well. Therefore, we restrict this study to these four rattail species.

\subsection{Commercial catches}

To complete a stock assessment, annual commercial catches, $C_{j t}^{\text {comm }}$ (tonnes), of rattails in the Chatham Rise hoki fishery are required by species $(j)$ and year $(t)$, from 1992 to 2012. Fishers are required to report catch, effort, landings, production and environment information to MPI, for species covered by the QMS. Estimated catch data are rough estimates of the catch (kg of each species) made by fishers as they fish. The top eight species by weight are required to be reported (up until 2011 the top 5 species by weight were required). However, no formal reported catches exist for any rattail species as they are not covered by the QMS and their catches are not required to be reported.

\subsubsection{Reconstructing catch histories}

In the initial stages of this project we planned to base the construction of species-specific rattail catch histories on observer data from commercial fisheries on the Chatham Rise, disaggregated using species proportions by year from the Chatham Rise trawl surveys. However, this option would have required significant work by NIWA to scale rattail observer totals to estimates of annual total catches (prior to disaggregation), and this was not feasible. We developed an alternative reconstruction method based on commercial effort and survey catches.

This method required survey data (catches and effort) that was available, and already supplied by NIWA, and commercial fishing effort. The commercial fishing data from the Chatham Rise is reported by fishing vessels to MPI. The MPI Catch Effort system stores information recorded by fishers on various MPI administrative forms. The Warehou database is a daily copy of the catch effort data. This information is primarily used for fisheries resource management and law enforcement purposes. Access to data from Warehou by clients external to MPI excludes access to any vessel or client identifying data. 
For the purposes of this study, permission was granted by MPI (17 April 2013) to access an existing dataset extracted from Warehou for NIWA (extract no. 8709, for contracts DEE2010002HOKB and MID20100201C). The dataset contains all effort variables for the CHAT4 reporting region (Figure 6), for landing, estimated or processing fishing events between 01 October 1989 and 30 September 2012 (note: fishing year e.g. $1997=$ 01 Oct 96 to 30 Sep 97), where hoki, hake or ling are specified in the catch. This extract was provided in a partially groomed state, where the data had been checked for errors using simple checking

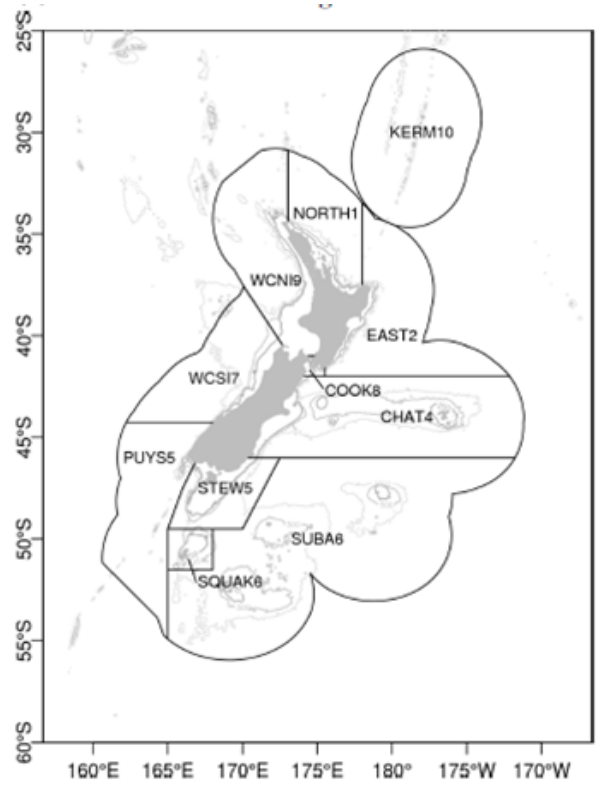

Figure 6: Reporting areas for trawl fisheries. Illustration from Abraham, Thompson and Oliver (2010) [1]. and imputation algorithms (S. Ballara, NIWA, personal communication, August 6, 2013.)

\section{Catch reconstruction method}

We propose a method of reconstructing catch history for rattail species utilising survey data; scaling the survey mean stratum catch/effort ratio for each species by commercial effort. (Note: the survey scales the stratum mean density (catch/area) by the survey area to give an estimated biomass for each species.). We make the assumptions that:

- in the same fishing year (01 Oct to $30 \mathrm{Sep}$ ), in the same fishing area (defined by a survey stratum) a unit of commercial fishing effort will catch the same species, in the the same proportions as a unit of survey fishing effort

- the characteristics of the catch from a unit of commercial fishing effort will not vary over the fishing year. (The survey is conducted in January each year.)

We first considered using the same indicator for commercial fishing effort as that used in the surveys, i.e. swept area, to scale survey stratum densities by commercial effort, i.e. $f_{i}=d_{i} w_{i}$, where $i$ indexes a trawling event 
(net deployed on ocean floor), $f$ is the area fished $\left(\mathrm{m}^{2}\right), d$ is the distance towed $(\mathrm{m})$ and $w$ is the width of a tow $(\mathrm{m})$. To be comparable with the survey trawls we would need to know the door spread and length of tow for each commercial trawl in the area. These data were not available in a readily usable form. Wingspread was available, but this relates to a narrower part of the gear. Converting wingspread to doorspread was considered unreliable due to lack of information regarding gear types used in each commercial tow. Also, distance towed could be estimated using the start and end position of each tow. However, this information is not available for all tows, and where available, could prove misleading, as (unlike the survey tows) the tow path may not be linear, i.e. the distance between the start and end location may not represent the actual length of the tow.

We then considered tow duration, i.e. the length of time (hours) the net is deployed on the ocean floor, as an indicator for fishing effort. Duration is recorded for each survey trawl and is also reported by commercial fishers for each trawl and recorded in the effort database. For commercial records the median and mean tow duration times were 4.4 and 4.6 hours, respectively. Recording errors may account for some of the larger duration values, especially those in excess of 10 hours. Replacing these records with the median of the remaining records decreases the total duration by $3.4 \%$. While it seems unlikely that a vessel will deploy a trawl net for periods in excess of a shift (e.g. 8 or 10 hours), in the absence of any definite information regarding likely tow times for the actual data we decided against any arbitrary replacement, and to use the data as it stands.

\section{Commercial fishing effort by stratum and fishing year}

In this report, from the effort data provided (453,744 records), we used trawl information on gear type, start date, start position and effort (duration of tow). We selected all records where:

- start date is between 01 October 1991 and 30 September 2012; and

- primary gear method equal to: BT, bottom trawl-single; BPT, bottom trawl-pair; MW, midwater trawl - single; MPT, midwater trawl-pair; ST, single trawl - bottom or mid not specified; $\mathrm{MB}$, midwater trawl fished on or close to the bottom (note, records only found for BT and $\mathrm{MB}$ in this selection); and

- tow start position (latitude and longitude) within polygon boundaries (Appendix E) specified for the core strata for survey trip TAN1001 (Appendix D). 
For the final selection of records $(M=204,312), 90 \%$ had BT as the primary gear method (MB, $8 \%$ and $\mathrm{MW}, 2 \%$ ). Also, $77 \%$ recorded the target species as hoki (73\%), hake or ling; with $14 \%$ recording scampi as the target species. Based on start date and start position, each selected record was assigned a fishing year (following the convention, e.g. 1993 for 01 Oct 92 to 30 Sep 93), and a stratum code based on the 20 amalgamated strata defined for the core survey (e.g. strata 010A and 010B combined to form 0010). A total commercial fishing effort $\left(E_{s t}^{\text {comm }}\right)$, as tow duration (hours), for each stratum $(s)$ within a fishing year $(t)$ could then be calculated as

$$
E_{s t}^{\text {comm }}=\sum_{i=1}^{m_{s t}} \text { duration }_{s t i} \quad \text { (hours) }
$$

where $s=(0001,0002, \ldots, 0020)$ and $t=(1992,1993, \ldots, 2012)$, and $m=$ the number of tow records for stratum $s$ and year $t$ (Appendix F). The series peaks at 65,512 hours in 1998 and declines to less than 40,000 hours from 2009 (Figure 7).

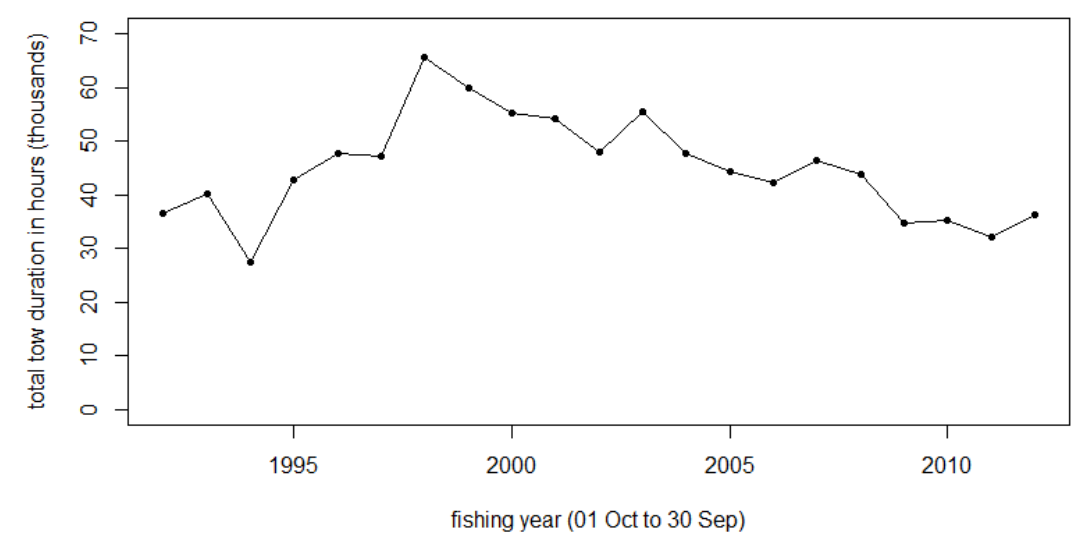

Figure 7: Commercial fishing effort as tow duration (hours) in the Chatham Rise core survey area where hoki, hake or ling are a recorded species, by fishing year 1992-2012.

Spatially, effort is concentrated in the western areas between 400 and 600 meters, particularly in strata 0007, 0008 and 0016; with the dominant area of effort moving to stratum 0019 (further east at 200-400m) after 2005 (Figure 13(a)). 


\section{Survey catch and effort, by species and stratum and fishing year}

For each survey sample, taken from a tow at a station within a stratum, the tow duration $D$ (hours) is available for the station, as is the catch weight $W$ (tonnes) for each species $j$ at that station. We used these measurements to calculate the mean stratum catch per effort $\left(C E_{s j t}^{\text {surv }}\right)$ by species and stratum and fishing year (in the same manner as mean stratum catch per area $A D_{s}$ is calculated for the survey, eq. (2.2)):

$$
\left.C E_{s j t}^{\text {surv }}=\frac{1}{h_{s t}}\left(\sum_{l=1}^{h_{s t}} \frac{W_{s j t l}}{D_{s t l}}\right) \quad \text { (tonnes } / \text { hour }\right)
$$

where $h=$ the number of stations for stratum $s$ and year $t$.

Spatially, the total sampling effort in each stratum (combined duration of station tows in each stratum) is more evenly distributed throughout the survey area (Figure 14(a))than that for the commercial effort (Figure 13(a)), which is to be expected when comparing a random survey to targeted commercial effort.

\section{Commercial catch by species and fishing year}

Using our estimates of commercial effort (eq. (2.5)) and survey catch and effort (eq. (2.6)), we then estimate the commercial catch $\left(C_{j t}^{\text {comm }}\right)$ for each species by year as

$$
\left.C_{j t}^{\text {comm }}=\sum_{s=0001}^{0200}\left(C E_{s j t}^{\text {surv }} \times E_{s t}^{\text {comm }}\right) \quad \text { (tonnes }\right)
$$

To gauge the plausibility of these catch estimates we compared our reconstructed catch histories for Javelinfish and for other mixed rattail species (Figure 8) against available estimates reported in studies by Livingston, Clark and Baird (2003) [52] and Ballara, O'Driscoll and Anderson (2010) [13] (Appendix C).

Livingston et. al. (2003) [52] looked at trends in bycatch of major fisheries on the CR for the fishing years from 1989-90 to 1998-99. To estimate catches of non-target species they used catch records from the scientific observer programme (SOP), noting that estimating total bycatch can be difficult because observer coverage of commercial vessels is inconsistent and only a small fraction of the tows are sampled. To estimate the total commercial catch for a bycatch species in a fishing year they took a ratio of the total observed bycatch to the total observed target species in the year and scaled this ratio by the reported total catch for the target species. 

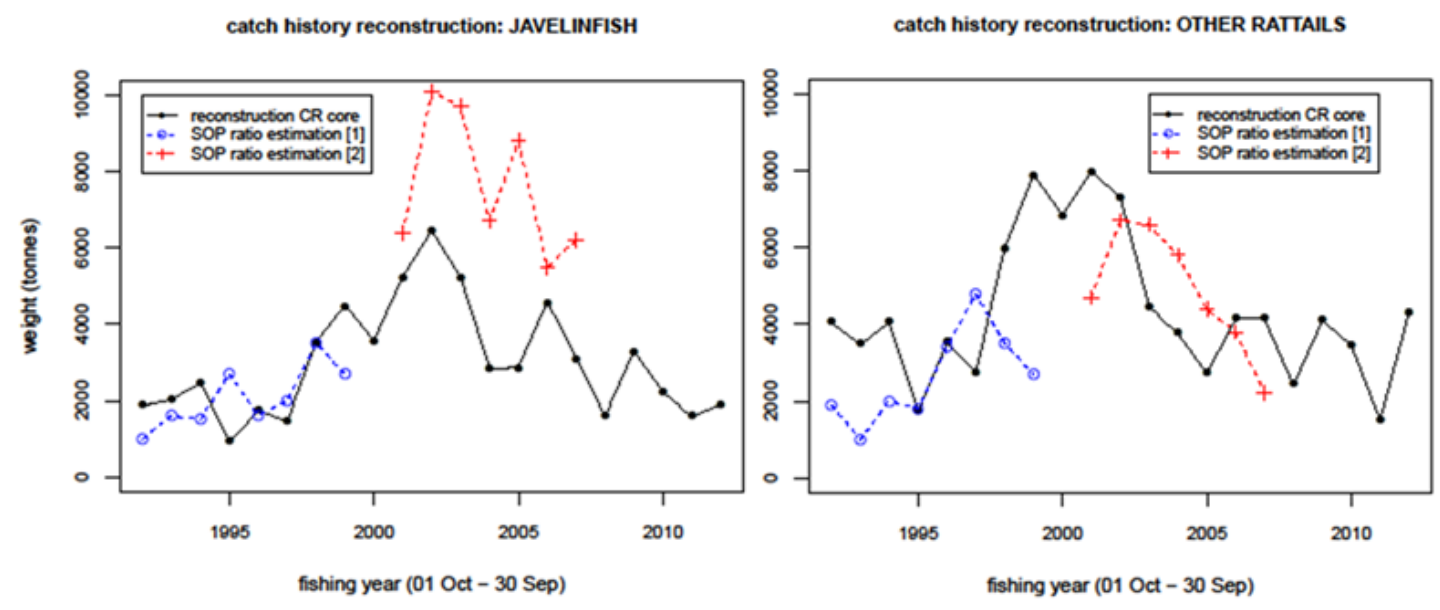

Figure 8: Bycatch estimates for rattail species Javelinfish (Lepidorhynchus denticulatus) (left) and other rattail species (right). Reconstruction CR core: this study, Chatham Rise core survey area. SOP ratio estimation(1): bycatch estimates for major fisheries in Chatham Rise, Livingston et. al. (2003) [52]. SOP ratio estimation(2): bycatch estimates for hoki, hake and ling fisheries in New Zealand waters, Ballara et. al. (2010) [13].
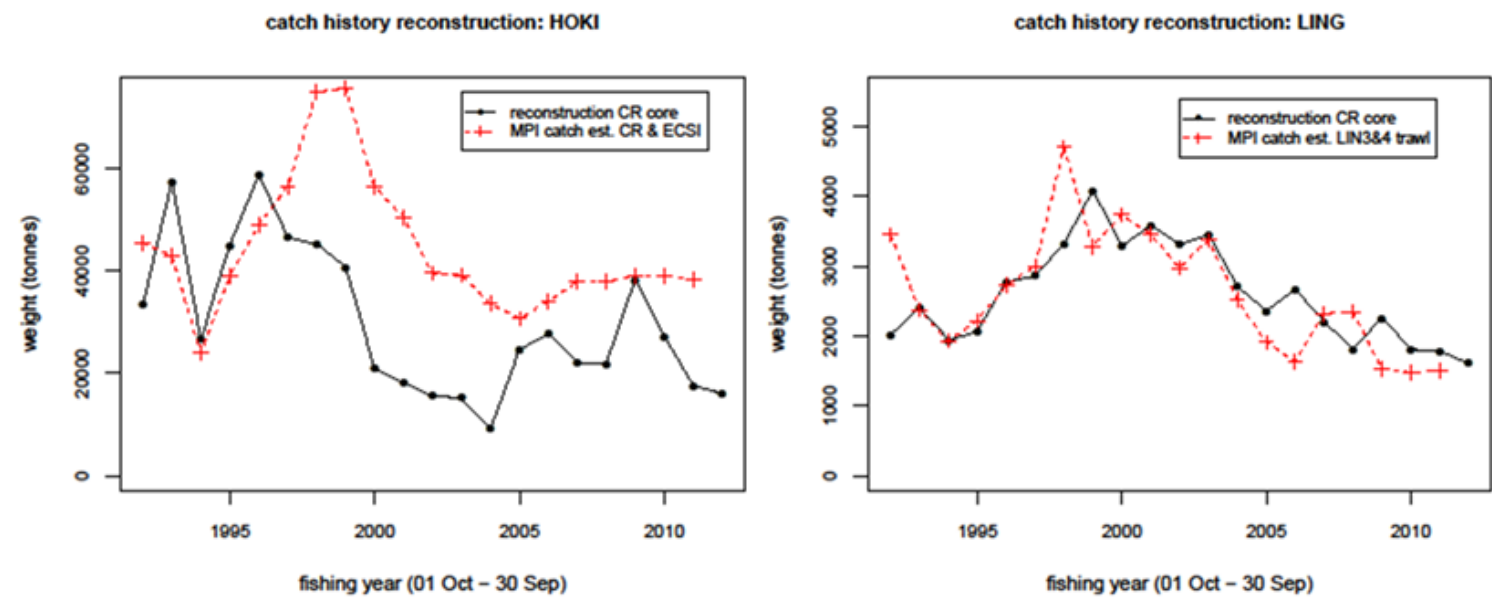

Figure 9: Left: Catch ( $t$ ) estimates for hoki (Macruronus novaezelandiae). Reconstruction CR core: this study, Chatham Rise core survey area. MPI catch est. CR \& ECSI: estimated total catch of hoki for the Chatham Rise and East Coast South Island non-spawning fishery areas, extracted from Table 3 (p. 395) in NZ MPI Fisheries Assessment Plenary Report (May, 2013) [67]. Right:Catch (t) estimates for ling (Genypterus blacodes). Reconstruction CR core: this study, Chatham Rise core survey area. MPI catch est. LIN3\&4 trawl: estimated catch of ling for the Chatham Rise trawl fishery area, extracted from Table 4 (p. 540) in NZ MPI Fisheries Assessment Plenary Report (May, 2013) [67]. 
The objective of the Ballara et. al. (2010) study was to estimate the catch (and discard rates) of non-target fish species in the hoki, hake and ling trawl fisheries for the fishing years 2003-04 to 2006-07 using data from the scientific observer programme and commercial fishing returns. After testing several ratio estimators, they used the ratio of observed catch to duration of observed tows (eq. (2.8)), scaled by total commercial tow duration to estimate the total yearly bycatch weight by species (eq. (2.9)):

$$
\begin{aligned}
& \widehat{B R}=\left(\sum_{i=1}^{m} n_{i}\right) /\left(\sum_{i=1}^{m} t_{i}\right) \\
& \widehat{B y C}=\widehat{B R} \times \widehat{T}
\end{aligned}
$$

where $m$ trawls are samples from a stratum, $n_{i}$ is the weight of the nontarget catch from the $i^{t h}$ trawl sampled, $T_{i}$ is the trawl duration for trawl $i$, and $\widehat{T}$ is the total trawl duration in the stratum; and with annual estimates of the bycatch of each species category calculated by summing the bycatch in each stratum (equation description from Ballara et. al. [13]). These estimates were for the total fishery, so the fishing areas included: Chatham Rise, West Coast South Island, Puyseger, Bounty, Cook Straight and SubAntarctic Islands.

Our catch estimates (Figure 8) are of the same order of magnitude as the estimates from these two studies. Livingston et al. (2003) used a catch ratio, whereas Ballara et. al. (2010) used an effort ratio as did we. In general, our estimates are less than those reported by Ballara et. al. (2010), especially for Javelinfish, and this is to be expected as that study extends to several areas outside the Chatham Rise. There is a noticeable peak in our estimates for the Other rattails group, from 1998 to 2002, that does not fit with the trends suggested by the other studies. Apart from checking that the estimates are not wildly out of range, it is difficult to take any further meaning from the comparisons or make explanations for similarities and differences without further study.

While it is difficult to test the plausibility of our estimates for rattails, we can use our method to generate catch histories for species where the catch histories are well documented for the Chatham Rise area. We have done this for hoki and ling (Figure 9). While our catch estimates are also of the same order of magnitude as the actual catches reported for these two species, in general we significantly underestimate the actual hoki catch. This result is not surprising as it is expected that the catch rates for commercial vessels that are actively hunting the target hoki species would be higher than those from the survey vessel (taking a random sample). Inter- 
estingly, the reconstructed catch history for ling is comparable with actual reported catches. Considering ling is also taken as bycatch in similar situations as rattail species it is a positive indication that there may be some merit in our method of catch history reconstruction for rattails.

It is important to note the limitations of the different methods of estimating bycatch. Those based on observer data are dependent on the coverage of observers to fishing trips, and "where observer coverage is poor, ratio estimates are likely to be poor" [52]. Also, rattails are not identified to species level by observers on board commercial fishing vessels. On the other hand, bycatch ratios produced from the Chatham Rise survey data can provide detail to species level for rattails, but may introduce bias. This would include bias associated with trawling as a random survey rather than as a commercial trawl targeting species such as hoki; also bias associated with the limited temporal coverage (January only) of the survey, whereas the commercial fishery operates over most of the year.

The trends in the generated catch histories for the four rattail species (Javelinfish, Bollon's rattail, Oliver's rattail, oblique banded rattail (data tabled in Appendix G)), are similar to the corresponding species survey biomass index (Figure 10), i.e. in general, as the index increases so does the catch (and vice versa). Although, for Oliver's rattail (COL) there is a period between 2003 and 2006 when the catches decline as the index increases.
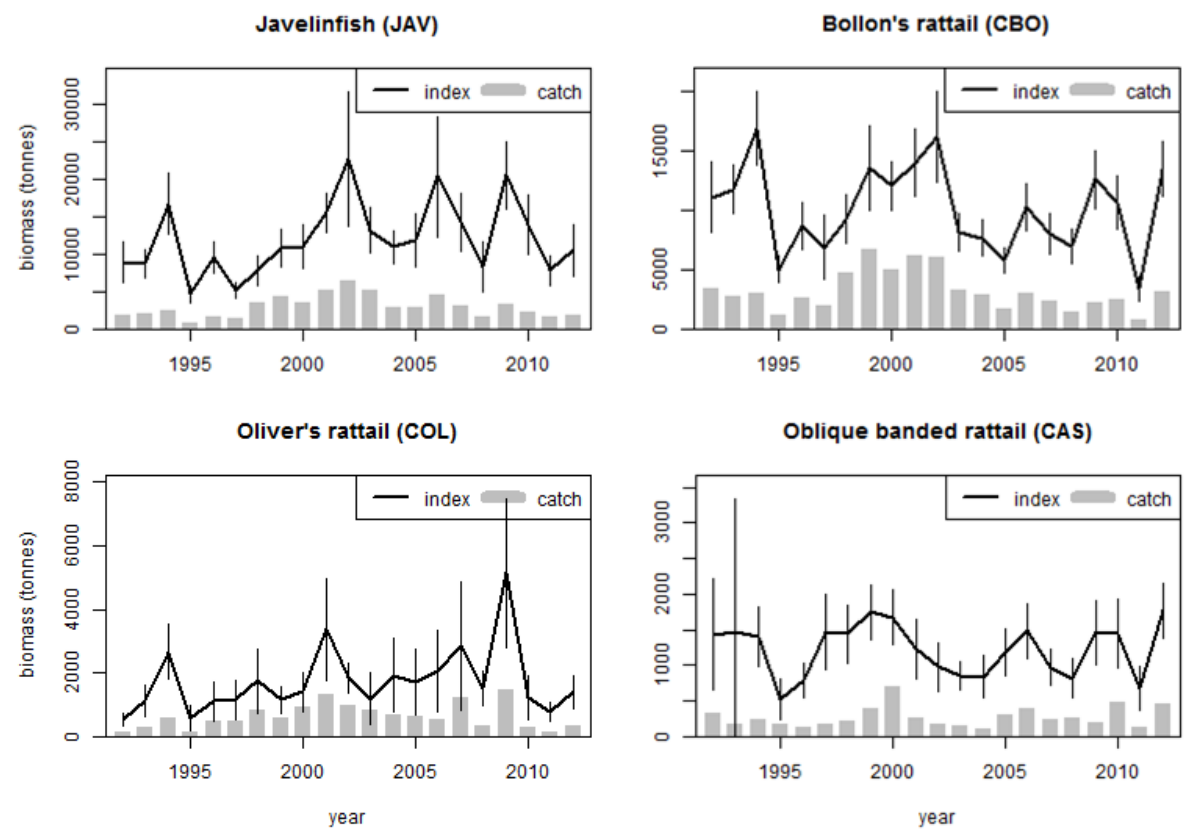

Figure 10: Reconstructed catch history for four rattail species, plotted together with the biomass index (including 95\% C.I.s), provided in NIWA summarised survey data. 
This result would be expected if the catch per unit effort ratio we used from the survey was scaled by a commercial effort that was constant across strata and over time. To determine if this is the case we constructed bubbleplots to investigate the spacial and temporal distribution of commercial and survey effort and catch (Figures 13 and 14).

Commercial effort (duration in hours) varies between strata and between fishing years (Figure 13(a)). The patterns of variation in commercial effort depicted in this bubbleplot are reflected in the bubbleplots of reconstructed catch histories of the four individual species (Figure 13(e-h)), where the spatial and temporal pattern in the commercial effort magnifies or reduces the pattern from the survey mean catch bubble plots (Figure 14(eh)). For example, if we compare the commercial effort for strata 0019 (more effort represented by larger bubbles) and 0020 (less effort represented by smaller bubbles), and we compare the survey mean catch for Javelinfish in these same strata - we see greater catch per hour in stratum 0020 than 0019. Yet, in the reconstructed catch for Javelinfish the catch is higher in strata 0019 than 0020, suggesting the commerical effort has had more of an influence on the magnitude of the estimated catches than the catch/effort ratio from the survey. This pattern scenario example for strata 0019 and 0020 is evident for the three other rattail plots and those for hoki and ling.

So, the pattern of our estimated catches for rattail species following the same trend as their survey abundance index may not be a result of our estimation process, which uses the survey abundance data. Our result for ling, tends to support this. Unlike the trend of estimated catches for the four rattail species, the generated catch history for ling shows a pattern of decreasing catches with increasing index (and vice versa) (Figure 11).

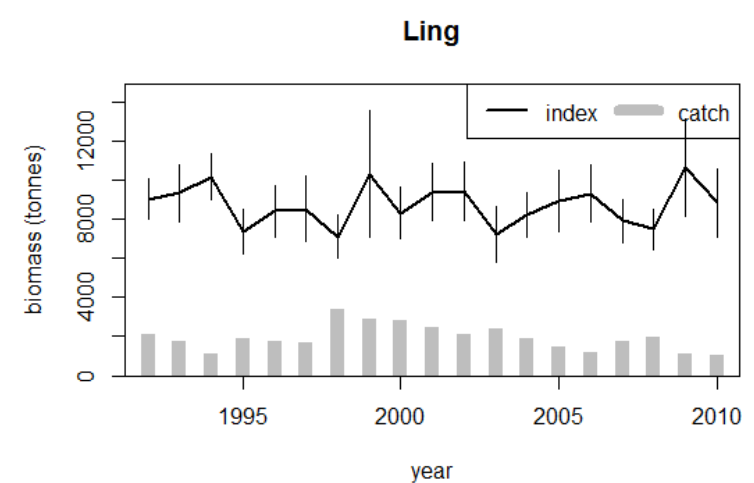

Figure 11: Reconstructed catch history for ling, plotted together with the biomass index (including 95\% C.I.s), provided in NIWA summarised survey data. 
This leads us to question what the cause of the pattern of catches for rattail species might be. We would expect, that if commercial catches are driving population abundance, then we would see some decline in abundance as catches increase and some "recovery" in abundance as catches decrease (as with ling). This pattern is more clearly depicted in the Namibian hake dataset from Hilborn and Mangel [46] (Figure (12). For the four rattail species we are investigating, catches may not explain the variation in abundance, i.e. population abundance may be driving the size of the catches.

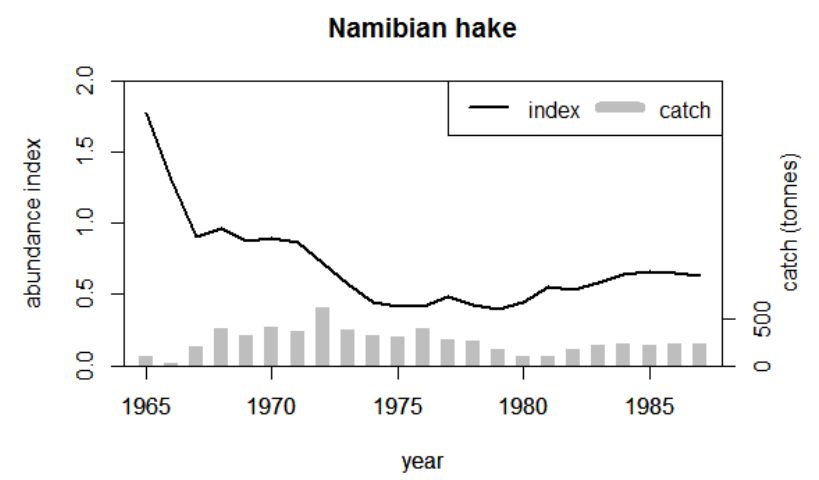

Figure 12: Catch history and abundance index for the Namibian hake dataset (plot uses data from page 240 of Hilborn and Mangel (1997) [46].

We are also interested in the patterns in the data because it is contrast in the data (variation in effort levels and stock sizes) that informs parameter estimation for models that attempt to define the stock-production relationship [54]. The commercial data provide information about the absolute scale of the fishery, while the abundance index provides information about the relative change in abundance over time [58], and the lack of contrast in these data may result in the failure of the stock assessment model to produce unbiased and precise parameter estimates [45]. Hilborn and Walters (1997) [47] describe good contrast in a data series where the data displays three distinct patterns: low stock size and fishing effort (provides information about the growth rate of a population); high stock size with low fishing effort (provides information about the scale of the population); and high fishing effort (provides information about the scale of the impact of fishery on the population). However, they also note, that it is unusual to obtain data with such widely divergent combinations of data points (due to stock sizes being serially corellated).

With the rattail data, where the patterns in the catches follow the patterns in the index, we may find that the lack of contrast does not enable our assessment models to produce reliable parameter estimates. 
(a) Commercial Effort (hours)

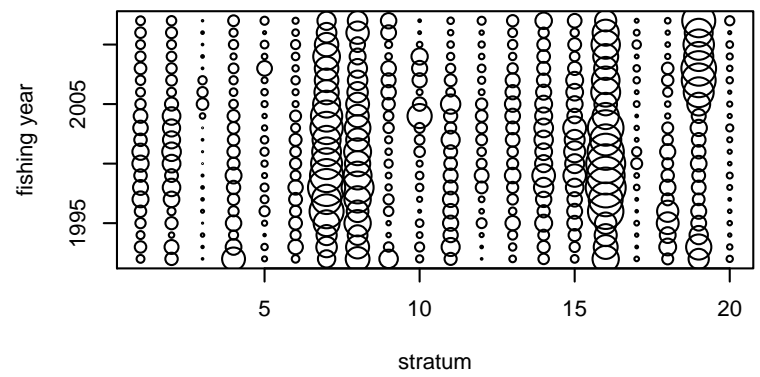

(c) Reonstructed catch (tonnes): HOKI

(d) Reonstructed catch (tonnnes): LIN
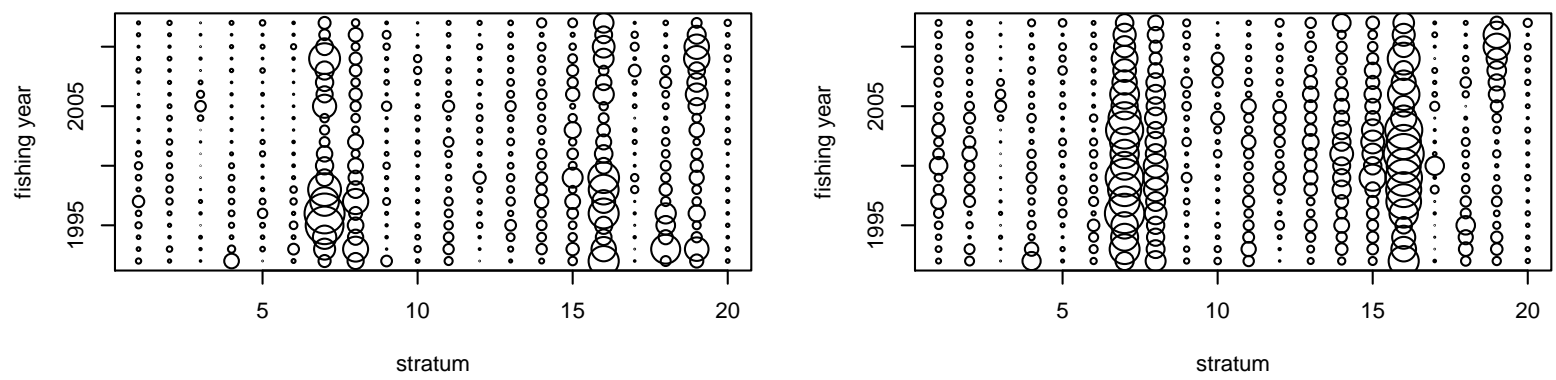

(e) Reonstructed catch (tonnes): JAV

(f) Reonstructed catch (tonnes): СBO
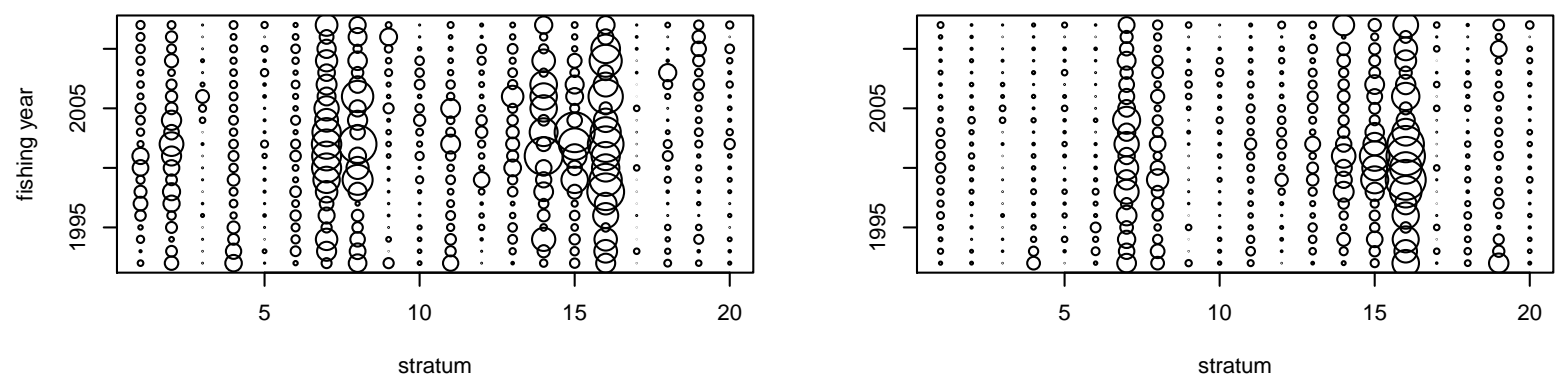

(g) Reonstructed catch (tonnes): COL

(h) Reonstructed catch (tonnes): CAS
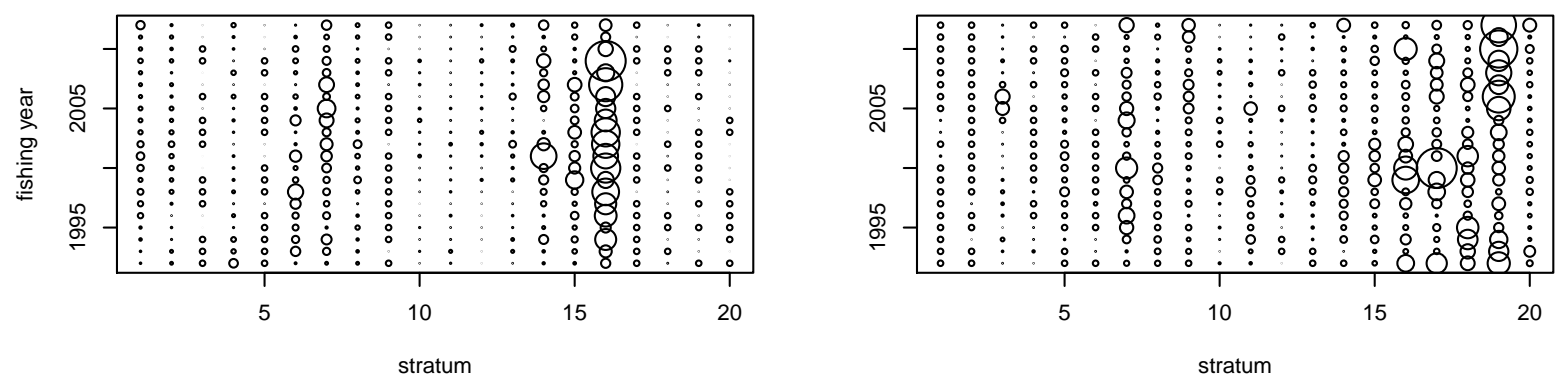

Figure 13: Spatial distribution of commercial catch and effort data in Chatham Rise core survey area: (a) Total commercial effort (hours) for bottom trawls where hoki are present in the catch, in each stratum, $E_{t s}^{\text {comm; }}$ (b-g) Reconstructed commercial catch (tonnes) by species in each stratum, $C_{j t s}^{\mathrm{comm}}$. (Size of circles comparable only within each plot). 
(a) Survey Effort (hours)

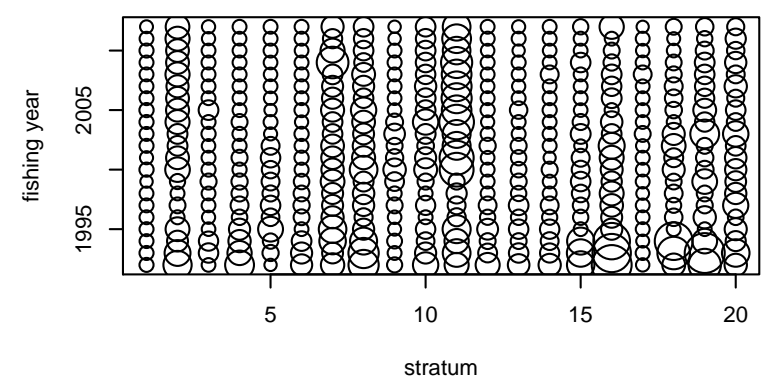

(b) Survey mean catch (tonnes): Hoki

(c) Survey mean catch (tonne): Ling

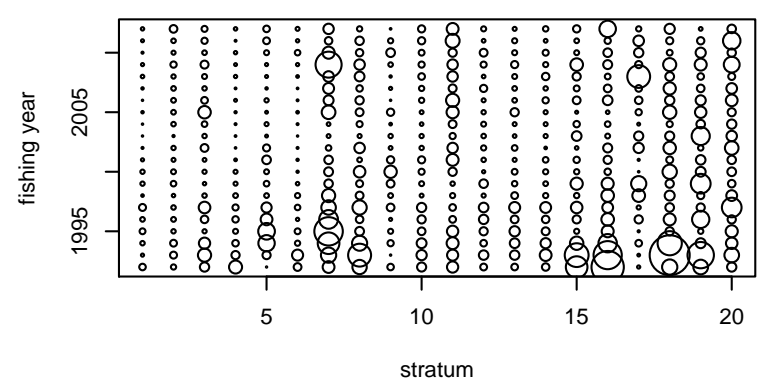

(d) Survey mean catch (tonnes) per hour: JAV

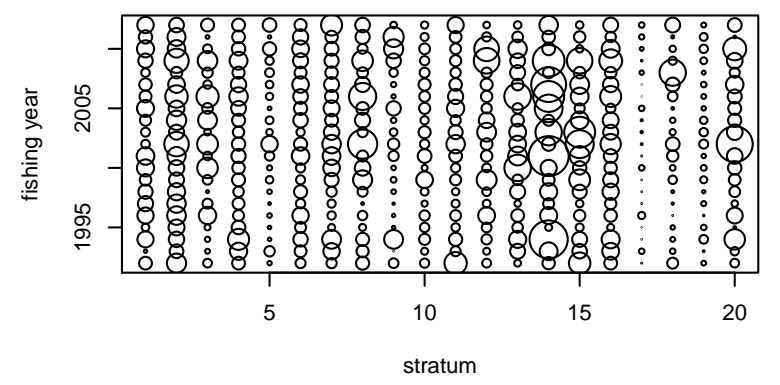

(f) Survey mean catch (tonnes) per hour: COL

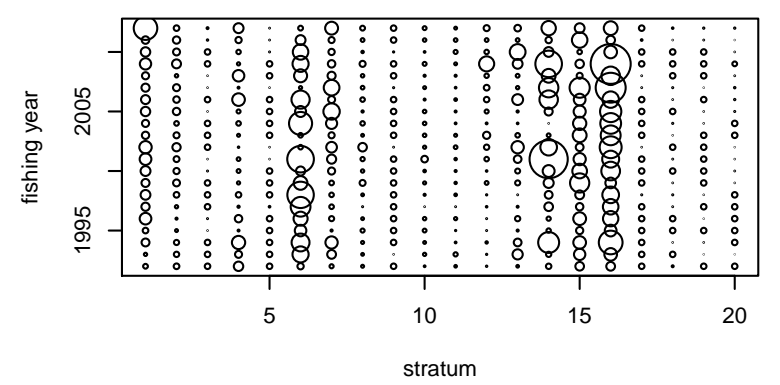

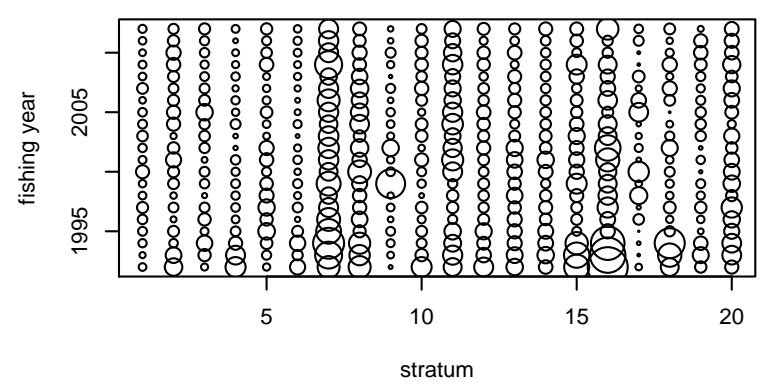

(e) Survey mean catch (tonnes) per hour: CBO

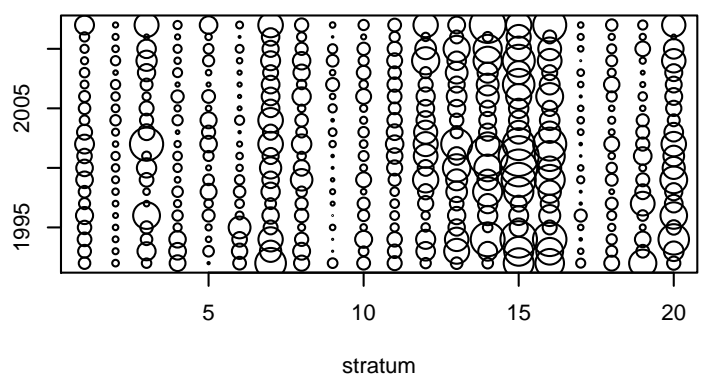

(g) Survey mean catch (tonnes) per hour: CAS

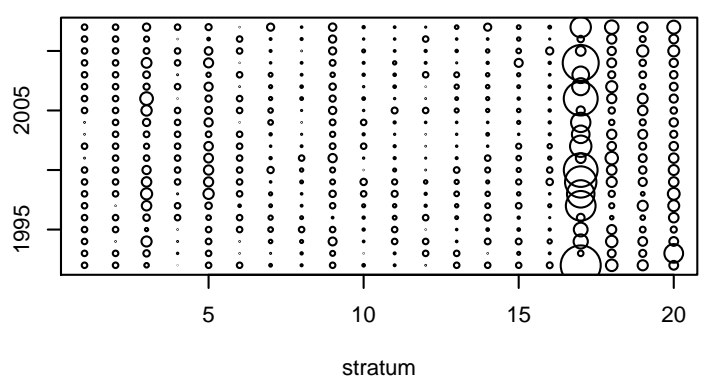

Figure 14: Spatial distribution of survey catch and effort data in Chatham Rise core survey area: (a) Total survey effort (hours) in each stratum, $E_{t s}^{\text {surv }}=\sum_{l=1}^{h_{t s}} D_{t s l}$; (b-g) Survey mean catch by species in each stratum $C E_{j t s}^{\text {surv }}$. (Size of circles comparable only within each plot). 


\section{Chapter 3}

\section{Stock assessment methods}

In fisheries, a stock assessment provides information to manage the exploitation of a fish stock. The key questions addressed in stock assessments are related to abundance (in numbers or biomass), such as historical trends and the current size of a fish stock, and how future levels of abundance may be affected by different fishing management strategies [35]. This is achieved by the modeling of fish population dynamics. The model is fitted using commercial fisheries data, and/or information collected independently to the commercial fishery.

Quinn [80] outlines the history and development of fisheries stock assessment methods in the 20th century. This was characterised by parallel development of population models in ecology and in fisheries, with statistical advances in fisheries science being almost exclusively found only in the fisheries literature. Quinn makes the particular point: "Few statisticians have been involved in fisheries modeling, and a whole class of methods, based on likelihood and Bayesian techniques are unlike anything found in the mainstream statistics literature."

Models available for stock assessments, describing changes in fish populations, are of two main types: those based on simple pooled-stock dynamics (biomass dynamic models), focusing on changes over time where there is no explicit accounting for age or size structure; and more complex ageand size-structured models, where dynamics are built on individual yearclasses [86]. Age-structured models are considered an improvement on the simpler biomass dynamics model as they can account for the varying levels of biomass contributed to a stock from the different sizes, growth rates and levels of reproduction of different aged animals [42]. This increased complexity can enable such models to better represent the natural processes of the population and, therefore, make better estimates about 
the fishery impact. But, these more complex models also require more data which is often not available.

The surplus production model (SPM) (which we describe in Section 3.1 below), a pooled-biomass dynamics model, has been used extensively in stock assessments [91] and is comprehensively documented in quantitative fisheries texts, e.g Quinn and Deriso [81], Hilborn and Walters [47], Haddon [42]. It is the simplest method available to model changes in the size of a fish population over time $[42,46]$ and is typically used when a lack of data precludes the use of more complex models [47, 75]. Notably, it does not involve age-structured modeling. Punt [78] notes that surplus production models "are virtually the only method for stock assessment in situations in which the only data available are a time series of catches and some index of abundance". The abundance index data provide information about the relative changes in abundance over time, whereas the catch data provide information about the absolute scale of the fishery [58], i.e. the model assumes the fishery catch is a forcing factor.

While surplus production models have been used in early stock assessment work, assessments in New Zealand for targeted and bycatch species of commercial value now make use of age-structured modeling techniques. For example, in the 2012 hoki stock assessment a range of data is used, collected from the fishery and from independent surveys (including acoustic biomass indices, proportions-at-age data, and proportions spawning data), with over 100 parameters estimated [62]. Of the 629 fish stocks in the QMS, 37 have quantitative assessments, and these tend to be for the high value species [14], such as hoki. Of the 83 species included in the MPI May 2013 Plenary Report [67] (an annual summary of stock assessment and stock status information for QMS species) 14 were based on a level 1 quantitative stock assessment using an age-structured model, e.g. hoki, hake, ling, paua, scampi, snapper. Other stocks were assessed at level 2 - partially quantitative, level 3 - qualitative, or level 4 - low information; using methods such as catch curve analysis, standardised CPUE (catch per unit effort), evaluation of trawl survey indices, or biomass estimated from quadrant surveys. For some species, the stock status was reported as unable to be assessed for various reasons, such as lack of data, lack of a proven method of assessment and more work required to validate key biological characteristics.

Extensive data collection programmes are usually supported by fisheries with high economic value [77]. However, for many fisheries the cost of monitoring and data collection necessary for a quantitative stock assess- 
ment cannot be met or is not warranted by the relatively low value of a species [85] (even if such species are taken as by-catch in a fishery targeting multiple high-value species). In these cases any potential for model complexity has outstripped the data available for parameter estimation [77]. For example, surplus production models have been used in stock assessments of New England winter flounder (eastern coast of North America) since the mid-1980's [41]. More complex age-structured models were attempted in the 1990's but did not persist as long-term, age-sampling programmes could not be maintained.

Also, while the type of data collected may enable the use of age-structured models, the information available in some of the data may not be adequate for estimating the greater number of parameters required by the more complex model. For example, Brodziac and Ishimura [18] use a surplus production model to assess the North Pacific swordfish population, which is considered to have high economic value in both commercial and recreational fisheries. Previously applied age-structured models had proved inadequate for assessing current population biomass for the swordfish population due to insufficient contrast in the fishery abundance index. In the context of parameter estimation, this contrast (i.e. historical variation in stock size and fishing pressure) is required to reliably estimate the model parameters [47] (and this also applies to surplus production models). Brodziac and Ishamura [18] found that, along with other improvements (e.g. consideration of the spatial structure of the population), the use of the more parsimonious surplus production model, with fewer parameters to estimate, improved the model fit to the abundance index.

The goal of the assessment work in this project is to develop a framework to differentiate and represent the population dynamics of different species of rattails that are subject to fishing pressure in fisheries that target other species (i.e. rattails are a bycatch species in the fishery). The main challenge for this project is the data status of these species in terms of: lack of species differentiation in commercial catch data; fisheries-independent annual biomass surveys not being specifically designed to measure these species; detailed biological information not available for all species; and possible lack of contrast in abundance index for some species. So, not only do we have insufficient data (data-poor situation) to attempt an agestructured model, the data we do have may not contain the contrast required (low-information situation) to adequately estimate parameters even in the least complex model.

For each species $j$ of rattail we apply a surplus production model to com- 
mercial catch data and fishery-independent scientific trawl survey biomass data.

\subsection{Surplus production model}

The surplus production model comprises two sub-models: the process model that describes population biomass dynamics; and the observation model that describes how abundance index observations relate to biomass predictions of the model [93]. In this section we describe the formulation of each sub-model, their combination in the full model, and the forms of error structure that determine methods of parameter estimation. For simplicity and convenience, the species subscript $j$ is dropped and what follows applies to any particular rattail species data to which the surplus production model is applied.

The process sub-model (eq. (3.1)) and the observation sub-model (eq. (3.2)), both of which are deterministic, form the basis of all biomass dynamics models

$$
\begin{aligned}
B_{t+1} & =B_{t}+f\left(B_{t}\right)-C_{t} \\
I_{t} & =q B_{t}
\end{aligned}
$$

where $B_{t}$ is the biomass (e.g. in tonnes) of a fish stock that is vulnerable to fishing (i.e. the exploitable biomass) at the start of period $t, f\left(B_{t}\right)$ is surplus production as a function of biomass, $C_{t}$ is the fishing catch in period $t, I_{t}$ is the index of abundance and $q$ is the catchability coefficient [75].

Surplus production models pool aspects of production (e.g. recruitment, growth and natural mortality), i.e.

$$
f\left(B_{t}\right)=\text { recruitment }_{t}+\text { growth }_{t}-\text { natural mortality }_{t}
$$

into the single production function $f\left(B_{t}\right)$, and the stock is considered an undifferentiated biomass where age- and size-structure are ignored. Based on the population ecology principle of density dependence, expressed as

$$
\frac{d B}{d t}=f(B)
$$

where $f^{\prime}(B) \leq 0$, the idea is that as populations grow (in the absence of fishing), they modify their environment through a web of multiple interactions (including other organisms and abiotic factors) which feeds back on their capacity to grow further [53]. That is, as $f(B)$ tends to 0 at high 
biomass, there is an environmental limit (carrying capacity) where populations are at the maximum conditions of their environment. The assumption is that the complex interactions and environmental feedbacks can be reduced to the dependence of demographic processes (recruitment, growth, natural mortality) on the population's own density. The function is a much simplified abstract of reality that has the advantage of not requiring large amounts of data and being relatively easy to implement [86].

While the function describing the production of biomass in any year $f(B)$ can take many forms [42], the classic models (based on the logistic function) are

$$
\begin{array}{rlrl}
f\left(B_{t}\right) & =r B_{t}\left(1-\frac{B_{t}}{K}\right) & & \text { Schaefer } 1954 \text { form }[84] \\
f\left(B_{t}\right)=r B_{t}\left(1-\frac{\log \left(B_{t}\right)}{\log (K)}\right) & & \text { Fox } 1970 \text { form }[29] \\
f\left(B_{t}\right)=\frac{r}{p} B_{t}\left[1-\left(\frac{B_{t}}{K}\right)^{p}\right] & & \text { Pella-Tomlinson 1969 form }[73]
\end{array}
$$

where $r$ is the intrinsic population growth rate parameter, $K$ is the carrying capacity (the average size of the biomass prior to exploitation, or in the absence of catch), and $p$ is a shape parameter $[42,47,75,86]$.

The concept of surplus production can be illustrated most simply with the Schaefer form as the function is symmetric about $\mathrm{K} / 2$ (the maximum rate of increase), with minimal or slow rates of increase when the population size approaches the asymptotic limits - at 0 (no population) and $\mathrm{K}$ (maximum population size) (Figure 15(a)). At these limits, the net production is zero. Surplus production occurs when the population size is less than the carrying capacity. As the logistic growth curve of the Schaefer model is symmetric, maximum surplus production of $r K / 4$ occurs at $B=K / 2$ [66] (Figure 15(b)).

The metric $B_{M S Y}$ (the level of population size which provides the maximum sustainable yield, MSY) has been used extensively in fisheries management as it is easily derived from the surplus production model, and is used to set target catches so as to maintain the population biomass at a level that yields a maximum rate of production. More recent management practices use $B_{M S Y}$ as a limit rather than a target $[56,57]$. 
(a) logistic growth curve

$\mathrm{dB} / \mathrm{dt}=\mathrm{rB}(1-\mathrm{B} / \mathrm{K})$

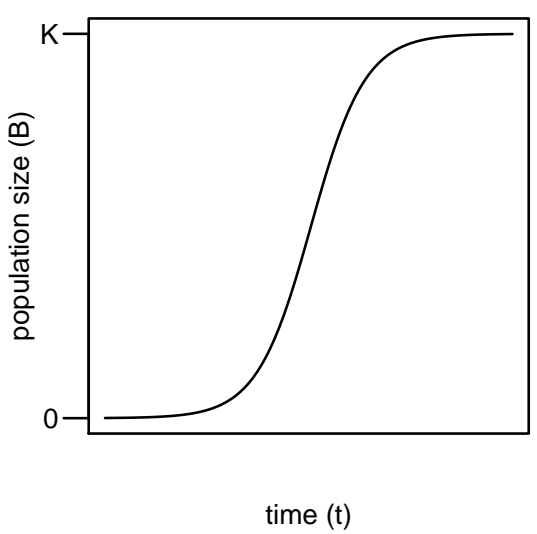

(b) surplus production

as population size increases

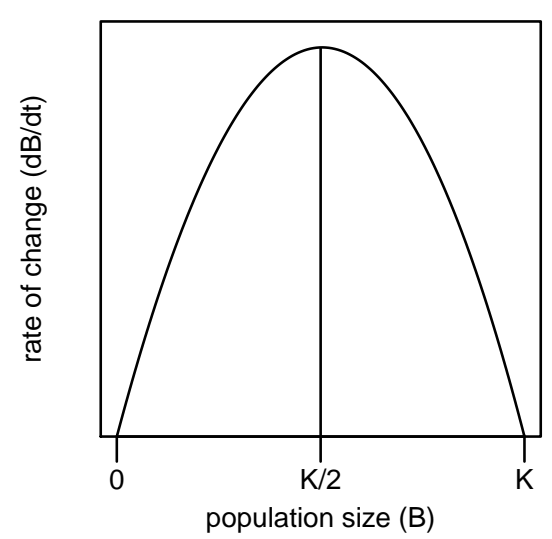

Figure 15: (a) Logistic growth curve of the Schaefer model; and (b) Rate of change $(d B / d t=f(B))$ in population biomass $(B)$ as biomass increases.

The Pella-Tomlinson and Fox forms of the production function were developed to allow for different asymmetric forms of the growth curve [75]. If $p>1$ the surplus production curve is skewed to the right, and maximum surplus production is obtained closer to the unfished biomass at $K$, and if $p<1$ the surplus production curve is skewed to the left [46]. At $p=1$ the Pella-Tomlinson form is equivalent to the Schaefer form, and the Fox form is the limit of the Pella-Tomlinson as $p$ tends to 0. However, the Fox form tended to be used in equilibrium applications of the surplus production model, which have been discounted since the early 1990's [46], and the Pella-Tomlinson form has been found to be difficult to fit. Prager [76] highlighted problems estimating parameters using the Pella-Tomlinson form as structural - related to the model being overly sensitive to outliers in noisy data (fishery datasets that tend to be short and noisy time-series) compared to the Schaefer form. The Schaefer is the standard form most frequently used, and we use the simpler Schaefer form.

Formulating logistic growth in discrete time steps using the Schaefer form as the production function, we define biomass dynamics in the process model as

$$
B_{t+1}=B_{t}+r B_{t}\left(1-\frac{B_{t}}{K}\right)-C_{t}
$$

In this way, detailed survival and recruitment dynamics are simplified into a few parameters that describe how the overall population changes from 
year to year and responds to exploitation. The model assumes constant population growth rate $r$, and carrying capacity $K$, and that the population is closed (no immigration or emigration). These are very strict assumptions and may not be very realistic. For example, growth rates depend on the growth in the size of survivors and the arrival of new fish (recruits), which may change over time in response to changes in the ecosystem. Migration may also occur for the same reason.

For the observation model the index of abundance is defined to have the property

$$
I_{t}=q B_{t}
$$

and assumes that $C_{t} / E_{t}$ is an index of abundance and hence that

$$
I_{t}=\frac{C_{t}}{E_{t}}=q B_{t}
$$

where $I_{t}$ is an index of abundance, $E_{t}$ is the fishing effort producing the catch in time $t$, and $q$ is the catchability coefficient. $E_{t}$ is not explicitly included in this model and expectations of $I_{t}$ are generated from model estimates of $B_{t}$ and $q$. The strong assumption in this model is that catch rates are linearly related to stock biomass $[42,82]$.

The catchability coefficient, $q$, can be thought of as a measure of the availability and vulnerability of a fish stock to the trawling process [36]. Catchability scales $B_{t}$ in the observation system. Values of $q$ depend on the units of $C, E$ and $B$. For example, if the observed biomass, $I$, from our survey is 1000 tonnes, and we know that $20 \%$ of the population is available to the trawling process $(q=0.2)$, then we determine the absolute biomass, $B$, in that year to be $1000 / 0.2=5000$ tonnes. While $q$ can be determined experimentally, this can be complex and the value may be poorly determined $[25,26,32]$. The catchability coefficient is generally treated as a nuisance parameter - required to scale the modeled stock biomass, $B$, to match the trends in the abundance index estimates, $\hat{I}$ [42]. As such it becomes an unknown parameter in the model, particular to each species, and is generally assumed to be constant over time and over the area fished and surveyed (i.e. no changes in fishing gear or vessel efficiency have taken place).

In summary, we make the following assumptions when applying the surplus production model:

- parameters $r, K$, and $q$ are constant over time; 
- $r$ is independent of age composition;

- the population is a single stock unit, that is closed (i.e. no immigration or emigration);

- fishing and natural mortality take place simultaneously;

- catch statistics are accurate (measured without error); and

- catch rate is proportional to biomass.

In reality, many of these assumptions may not be met. The main criticism leveled at the use of the surplus production in stock assessments is its lack of biological realism due to age-structure not being included. This is also highlighted as the advantage of this model, providing a simpler modeling tool that requires a less complex dataset. This advantage allows for stock assessments where more complex data collection systems are unavailable, although it is recommended that the model be used critically in light of the assumptions [17].

Errors are associated with both the observation and the process model. It is commonly assumed that these errors follow a Normal distribution with mean 0 and variance $\sigma^{2}$ [42], i.e.

$$
\begin{aligned}
B_{t+1} & =\left(B_{t}+r B_{t}\left(1-\frac{B_{t}}{K}\right)-C_{t}\right) e^{\varepsilon_{t}^{v}} \\
I_{t} & =q B_{t} e^{\varepsilon_{t}^{u}}
\end{aligned}
$$

where

$$
\varepsilon_{t}^{v} \stackrel{\mathrm{iid}}{\sim} N\left(0, \sigma_{v}^{2}\right), \quad \varepsilon_{t}^{u} \stackrel{\mathrm{iid}}{\sim} N\left(0, \sigma_{u}^{2}\right)
$$

Considering observation errors first, $\varepsilon_{t}^{u}$ has the density function

$$
f\left(\varepsilon_{t}^{u}\right)=\frac{1}{\sqrt{2 \pi \sigma_{u}^{2}}} \exp \left(-\frac{1}{2 \sigma_{u}^{2}}\left(\varepsilon_{t}^{u}\right)^{2}\right)
$$

To determine the density function for $I_{t}$ we consider $I_{t}$ as a function $h($. of $\varepsilon_{t}^{u}$. That is, we let

$$
I_{t}=h\left(\varepsilon_{t}^{u}\right), \quad \text { where } h\left(\varepsilon_{t}^{u}\right)=q B_{t} e^{\varepsilon_{t}^{u}}
$$

The inverse of $h($.$) is given by$

$$
h^{-1}\left(I_{t}\right)=\varepsilon_{t}^{u}
$$


where

$$
h^{-1}\left(I_{t}\right)=\varepsilon_{t}^{u}=\log \left(I_{t}\right)-\log \left(q B_{t}\right)
$$

Then, using the change of variables theorem, the probability density function $g\left(I_{t}\right)$ is specified by the relation

$$
\begin{aligned}
g\left(I_{t}\right) & =f\left[\left(h^{-1}\left(I_{t}\right)\right]\left|\frac{d h^{-1}\left(I_{t}\right)}{d I_{t}}\right|\right. \\
& =\frac{1}{\sqrt{2 \pi \sigma_{u}^{2}}} \exp \left(-\frac{1}{2 \sigma_{u}^{2}}\left[\log \left(I_{t}\right)-\log \left(q B_{t}\right)\right]^{2}\right)\left|\frac{1}{I_{t}}\right| \\
& =\frac{1}{I_{t} \sqrt{2 \pi \sigma_{u}^{2}}} \exp \left(-\frac{1}{2 \sigma_{u}^{2}}\left[\log \left(I_{t}\right)-\log \left(q B_{t}\right)\right]^{2}\right)
\end{aligned}
$$

This is the density function of a log-Normal random variable, therefore

$$
\log \left(I_{t}\right) \sim N\left(\log \left[q B_{t}\right], \sigma_{u}^{2}\right)
$$

The process errors $\varepsilon_{t}^{v}$ also have the density function

$$
f\left(\varepsilon_{t}^{v}\right)=\frac{1}{\sqrt{2 \pi \sigma_{v}^{2}}} \exp \left(-\frac{1}{2 \sigma_{u}^{2}}\left(\varepsilon_{t}^{v}\right)^{2}\right)
$$

and the probability density function $g($.$) for the biological process is gen-$ erated in the same manner which gives

$$
g\left(B_{t+1}\right)=\frac{1}{B_{t+1} \sqrt{2 \pi \sigma_{v}^{2}}} \exp \left(-\frac{1}{2 \sigma_{v}^{2}}\left[\log \left(\frac{B_{t+1}}{B_{t}+r B_{t}\left(1-\frac{B_{t}}{K}\right)-C_{t}}\right)\right]^{2}\right)
$$

and, therefore

$$
\log \left(B_{t+1}\right) \sim N\left(\log \left[B_{t}+r B_{t}\left(1-\frac{B_{t}}{K}\right)-C_{t}\right], \sigma_{v}^{2}\right)
$$

Observation error can result from sampling error and (random) variation in the availability of the population to the sampling gear, and sources of process error can include variability in recruitment, growth and natural mortality [78]. As such it makes sense that both forms of error should be included in the model [42]. However, due to difficulties encountered when including both sets of errors, observation-error only models tend to be used $[47,42,76,91]$. While process-error only models have been shown to provide less precise estimates of model parameters, observation-error only models have also been shown to provide biased and imprecise estimates when process-error is also present [78]. 
The recommended method for fitting fisheries models to data is timeseries fitting [17, 42,46]. The most widely used and simple method of estimating parameters for the surplus production model is to apply maximum likelihood estimation to the observation-only model [47]. We apply this method to our data, described in the next chapter (Chapter 4).

More complex methods have been explored for fitting models with both error types, such as: total-error and state-space models. The total-error model is similar to the observation-error only model and specifies some relationship between the process and observation error which could be a known ratio or based on some arbitrary assumption. State-space models combine a stochastic model for population dynamics (state) with a stochastic model for observations and have been fitted using such method as the Kalman Filter and the Bayesian approach [23]. Kalman filters (derived by applying Bayes theorem to recursively update linear combinations of normally distributed random variables [80]) require an extension to apply to the nonlinear Schaefer production model [78] and are considered generally intractable [42]. We apply a state-space method utilising the Bayesian approach [60,66], described in Chapter 5. 


\section{Chapter 4}

\section{Maximum likelihood estimation of observation model parameters}

This chapter presents the maximum likelihood estimation (MLE) method and estimation results for the observation model parameters. Model fitting and estimation is done separately for each species.

\subsection{Maximum likelihood estimation}

With the observation-error only model the population dynamics are assumed to be deterministic, i.e. $\sigma_{v}^{2}=0 \Rightarrow \varepsilon_{t}^{v}=0 \forall t$, and that all errors occur in the relationship between stock biomass and the index of abundance [75]. The likelihood of the observed abundance index for time $t$ from eq. (3.18) on page 33 , given the biomass $B_{t}$, is

$$
\begin{aligned}
L\left(q, B_{1}, \sigma_{u} \mid I_{t}\right) & =g\left(I_{t} ; q, B_{1}, \sigma_{u}\right) \\
& =\frac{1}{I_{t} \sqrt{2 \pi \sigma_{u}^{2}}} \exp \left(-\frac{1}{2 \sigma_{u}^{2}}\left[\log \left(I_{t}\right)-\log \left(q B_{t}\right)\right]^{2}\right)
\end{aligned}
$$

The probability of seeing the data actually collected given the parameters $P\left(I_{t} \mid q, B_{t}, \sigma_{u}\right)$ can be viewed as the likelihood of the parameters given the data $L\left(q, B_{t}, \sigma_{u} \mid I_{t}\right)$. The maximum likelihood estimates (MLE) of the parameters are those values of the parameters at which the likelihood is at its maximum value. That is, the MLEs are the parameter values at which the probability of observing the sample collected is at its highest. MLE is defined as

$$
\arg \sup _{q, B_{1}, \sigma_{u}} L\left(q, B_{1}, \sigma_{u} \mid I_{t}\right)
$$


or equivalently

$$
\arg \min _{q, B_{1}, \sigma_{u}}-L\left(q, B_{1}, \sigma_{u} \mid I_{t}\right)
$$

While it is likely that successive observations are correlated (since the size of the population, $B_{t}$, is dependent on structural changes in the population over time through the surplus production model [81]), the joint density of the sample makes the implicit assumption of conditional independence between observations in different years. Therefore, the full likelihood function for the observation model is

$$
L\left(r, K, q, B_{1}, \sigma_{u} \mid I_{t}, C_{t}\right)=\prod_{t=1}^{n} \frac{1}{I_{t} \sqrt{2 \pi \sigma_{u}^{2}}} \exp \left(-\frac{\left[\log \left(I_{t}\right)-\log \left(q B_{t}\right)\right]^{2}}{2 \sigma_{u}^{2}}\right)
$$

where $n$ is the total number of years (i.e. $n=21$, for 1992 to 2012) and

$$
B_{t}=B_{t-1}+r B_{t-1}\left(1-\frac{B_{t-1}}{K}\right)-C_{t-1}
$$

and the negative log-likelihood equals

$$
\begin{aligned}
& -\ell\left(r, K, q, B_{1}, \sigma_{u} \mid I_{t}, C_{t}\right) \\
= & -\log \left[\prod_{t=1}^{n} \frac{1}{I_{t} \sqrt{2 \pi \sigma_{u}^{2}}} \exp \left(\frac{-\left[\log \left(I_{t}\right)-\log \left(q B_{t}\right)\right]^{2}}{2 \sigma_{u}^{2}}\right)\right] \\
= & -\sum_{t=1}^{n} \log \left[\frac{1}{I_{t} \sqrt{2 \pi \sigma_{u}^{2}}} \exp \left(\frac{-\left[\log \left(I_{t}\right)-\log \left(q B_{t}\right)\right]^{2}}{2 \sigma_{u}^{2}}\right)\right] \\
= & \sum_{t=1}^{n} \log \left(I_{t}\right)+\frac{n}{2} \log (2 \pi)+\frac{n}{2} \log \left(\sigma_{u}^{2}\right)+\sum_{t=1}^{n} \frac{\left[\log \left(I_{t}\right)-\log \left(q B_{t}\right)\right]^{2}}{2 \sigma_{u}^{2}}(4) \\
= & \frac{n}{2} \log \left(\sigma_{u}^{2}\right)+\sum_{t=1}^{n} \frac{\left[\log \left(I_{t}\right)-\log \left(q B_{t}\right)\right]^{2}}{2 \sigma_{u}^{2}}+\text { const. }
\end{aligned}
$$

as the first two terms of eq. (4.6) are constant with respect to the unknown parameters. 


\subsection{Estimation procedure}

The unknown parameters $r, K, q, \sigma_{u}$ and $B_{1}$ will be estimated using maximum likelihood estimation. The likelihood will be maximised numerically using the opt im package in R (note: this package finds the MLE by searching for the value that minimises $-\log L)$.

With multiple ( $k=4$ or 5 ) parameters to estimate, the likelihood is a surface in $k$-dimensional space, where the MLE is found by searching over the surface. This is done analytically by equating to zero the partial derivatives of the log-likelihood with respect to each parameter, then finding a solution to these simultaneous equations. However, such an analytic solution for our likelihood is not available, and we need to find MLEs for the parameters numerically. We do this with the opt im package in R, using three different built-in optimisation techniques: 1) Nelder-Mead (NM) is a derivative-free minimization algorithm and is optim's default; 2) LBFGS-U is a "quasi-Newton" method that uses the general idea of calculating derivatives to iteratively approximate the root of the derivatives, and includes upper and lower bounds for each parameter; and 3) Simulated annealing (SANN) (a Metropolis algorithm) is a stochastic global optimizer that relies on adding random noise to the likelihood surface as a way of avoiding being trapped at one particular maximum and is useful when the likelihood surface may have more than one local maximum [15]. We also use the met ropsB (MSB) optimising function (another variant of the Metropolis algorithm) from the embrook package in R [16].

We will also need to estimate the starting biomass $B_{1}$ as our data do not extend back to the beginning of the fishery. Hilborn and Walters [46] note that there is usually very severe parameter confounding between $r, K$, and $q$, and that including $B_{1}$ as another parameter to estimate makes matters worse. We will fit the model both with $B_{1}=K$ and also with estimating $B_{1}$.

To reduce the dimension of the parameter space for numerical optimsation, the maximum likelihood estimator of the standard deviation $\sigma_{u}$ will be determined analytically using

$$
\widehat{\sigma}_{u}=\sqrt{\frac{1}{n} \sum_{t=1}^{n}\left[\log \left(I_{t}\right)-\log \left(q B_{t}\right)\right]^{2}}
$$

an approach used by Polacheck et. al. (1993) [75]. Substituting eq. (4.8) into eq. (4.7) as described in Haddon [42], we have, along the profile where 
$L$ is maximised over $\sigma_{u}$ :

$$
\begin{aligned}
& \left.-\ell\left(r, K, q, B_{1}, \widehat{\sigma}_{u} \mid I_{t}, C_{t}\right)\right] \\
= & \frac{n}{2} \log \left(\widehat{\sigma}_{u}^{2}\right)+\frac{\left(\sum_{t=1}^{n}\left[\log \left(I_{t}\right)-\log \left(q B_{t}\right)\right]^{2}\right)}{\left(\frac{2}{n} \sum_{t=1}^{n}\left[\log \left(I_{t}\right)-\log \left(q B_{t}\right)\right]^{2}\right)}+\text { const. } \\
= & \frac{n}{2}\left(\log \left(\widehat{\sigma}_{u}^{2}\right)+1\right) \\
= & \frac{n}{2}\left(\log \left(\frac{1}{n} \sum_{t=1}^{n}\left[\log \left(I_{t}\right)-\log \left(q B_{t}\right)\right]^{2}\right)+1\right)
\end{aligned}
$$

Each of the four optimisation algorithms is applied (separately) to the two estimation procedures (Proc.):

Proc. A estimates $r, K, q, \sigma_{u} \quad$ (assume $B 1=K$ );

Proc. B estimates $r, K, q, \sigma_{u}, B_{1}$

We applied these model setups to the data of the four rattail species according to the algorithm described in Hilborn and Mangel [46] (which they applied to a Namibian hake dataset), to find values of the parameters that minimised the negative log-likelihood:

1. Input the catch and index data $\left(I_{t}, C_{t}\right)$

2. Input starting values for the parameters to be estimated $\left(r, K, q, \sigma_{u}, B_{1}\right)$

3. Estimate values for $B_{t}$ using eq. (3.1)

4. Estimate values of $\sigma_{u}$ using eq. (4.8)

5. Calculate the negative log-likelihood: using eq.(4.9)

6. Iteratively apply steps $4-5$ based on updated values of the parameters until the optimiser converges, and the minimum $-\log L$ value has been found. 


\subsection{Model assessment}

To assess the model fit we use Akaike Information Criteria (AIC) to compare models, profile likelihoods to look at uncertainty around individual estimated parameter values, and bootstrapped confidence intervals to look at the uncertainty related to fitted index.

Information criteria methods such as AIC assess the balance between the relative quality of fit (the variation in the data that the model accounts for) and the number of parameters fitted [42]. We can compare models using

$$
A I C=-2 \log L+2 k
$$

where $L$ is the maximum likelihood value and $k$ is the number of parameters estimated by the model; and where smaller values represent better overall fits. We also calculate the AIC with a finite-sample size correction, the AICc, recommended for small sample sizes, e.g. when $n / k<40$ [15],

$$
A I C c=A I C+\frac{2 k(k+1)}{n-k-1}
$$

Profile likelihoods can give some idea of the shape of the likelihood surface for single parameters. To gauge the uncertainty of parameter estimates we plot the likelihood profiles for the two main biological parameters, $r$ and $K$, by setting a range of fixed values for the parameter of interest and then for each fixed value optimising the likelihood with respect to all the other parameters. An approximate $95 \%$ univariate confidence interval for the parameter is based on the likelihood ratio test where twice the negative log-likelihood ratio (the deviance) is approximately chi-squared distributed (i.e. $-2 \log (L)_{\max } \sim \chi_{1}^{2}$ ), with 1 degree of freedom (for the one restricted parameter) [10]. The confidence limit cuts the likelihood profile at $-2 \log (L)_{\max }+\chi_{1,0.95}^{2}$, and upper- and lower- confidence bounds are the values of the fixed parameter at this negative log-likelihood limit.

Confidence intervals (CI) for model parameters and the estimated index $\hat{I}_{t}$ are obtained using a bootstrap procedure that resamples residuals from the estimated index to create a new time series of an "observed" index. Due to the time series nature of our data, resampling the residuals, rather than the observed data (as is usual when bootstrapping), allows for the order of the data to be maintained [76]. We use a bootstrap method that combines the ordered estimates of the index from the original model fit with residuals chosen at random from that fit [28]. 
That is, we calculate fitted value $\hat{I}_{t}$ using model equations (3.1) and (3.2), and the estimated parameters: $\hat{r}, \hat{K}, \hat{q}, \hat{B}_{1}$ and $\hat{\sigma}_{u}$. We then calculate the residuals as the ratio (errors are lognormal [42]) of the observed index to the expected index: $I_{t} / \hat{I}_{t}$. Then, calculate the bootstrapped index $\hat{I}_{t}^{*}$ as the product of the fitted index and a random sample with replacement of the residuals, i.e.

$$
\log \left(\hat{I}_{t}^{*}\right)=\log \left(\hat{I}_{t}\right)+\left[\log \left(I_{t}\right)-\log \left(\hat{I}_{t}\right)\right]^{*}
$$

The bootstrapped index $\hat{I}_{t}^{*}$ is now the new time series of the observed index. Confidence intervals are estimated by generating many $(J)$ bootstrap samples $\left(\hat{I}_{t}^{*}\right)_{j}$, refitting the model and re-estimating the model parameters $\left(\hat{r}^{*}, \hat{K}^{*}, \hat{q}^{*}, \hat{B}_{1}^{*}\right.$ and $\left.\hat{\sigma}_{u}^{*}\right)$ for each bootstrap sample, and taking the 0.025 and 0.975 percentile values (for a $95 \% \mathrm{CI}$ ) of the sorted bootstrapped parameter estimates. The bootstrapped parameter estimates at these upper and lower bounds can then be used in the model equations to generate the confidence bounds for the estimated index $\hat{I}_{t}$.

Also, we calculate the bootstrap median bias as the proportion of resampled values that are less than or equal to the value of our maximum likelihood estimate for a parameter, where a proportion of 0.5 indicates no bias [27]. As a rough guide we would consider a median bias less than 0.35 or greater than 0.65 as an indication of a problem, and that we need to find a less biased parameter estimate. 


\subsection{Results}

\subsubsection{Namibian hake data}

As a test, we applied our method to the Namibian hake dataset from Hilborn and Mangel [46] (Figure 12 on page 22) where they have published results using maximum likelihood estimation. The results from our model fits are listed in Table 4.1, along with the parameter estimates from Hilborn and Mangel.

Table 4.1:

Results of model fits for Namibian hake data

\begin{tabular}{|c|c|c|c|c|c|c|c|c|c|}
\hline \multirow{2}{*}{$\begin{array}{c}\text { procedure } \\
\text { type }\end{array}$} & \multirow{2}{*}{$\begin{array}{l}\text { optim } \\
\text { method }\end{array}$} & \multirow{2}{*}{$\begin{array}{c}\text { min. value } \\
-\log L\end{array}$} & \multicolumn{3}{|c|}{ parameter estimates: } & \multirow[b]{2}{*}{$\sigma_{u}$} & \multirow[b]{2}{*}{$B_{1}$} & \multicolumn{2}{|c|}{ information criteria: } \\
\hline & & & $r$ & $K$ & $q$ & & & AIC & $\mathrm{AICc}$ \\
\hline \multicolumn{3}{|c|}{ Hilborn \& Mangel model fit [46] } & 0.39 & 2709 & 0.00045 & 0.1200 & & & \\
\hline \multirow[t]{4}{*}{$\mathrm{A}$} & N-M & -33.03301 & 0.50 & 2275 & 0.00054 & 0.1442 & & & \\
\hline & L-BFGS-U & -35.17865 & 0.39 & 2737 & 0.00043 & 0.1313 & & -64.357 & -63.094 \\
\hline & SANN & -33.62878 & 0.35 & 2993 & 0.00036 & 0.1609 & & & \\
\hline & MSB & -28.51339 & 0.23 & 4082 & 0.00025 & 0.1753 & & & \\
\hline \multirow[t]{4}{*}{ B } & N-M & -11.82359 & 0.49 & 10908 & 0.00006 & 0.3627 & 10908 & & \\
\hline & L-BFGS-U & -0.86776 & 0.48 & 11452 & 0.00009 & 0.5241 & 11443 & & \\
\hline & SANN & -34.49025 & 0.37 & 2779 & 0.00043 & 0.1596 & 2726 & -60.981 & -58.758 \\
\hline & MSB & -32.83409 & 0.31 & 3258 & 0.00034 & 0.1671 & 3239 & & \\
\hline
\end{tabular}

Both procedure types produce parameter estimates that are consistent with those of Hilborn and Mangel [46] (including plausible estimates for $B_{1}$ ). Compared to Proc. B, Proc. A gave lower AIC and AICc values across all optimisation methods, with the best results obtained using the L-BFGS-U algorithm. This is to be expected, and is in keeping with Hilborn and Walters [47] that including $B_{1}$ as an unknown to be estimated reduces model performance.

However, both procedures produced different results for different optimisation methods, suggesting the likelihood surface is difficult to investigate. This could be due to the surface being "flat" or because the surface has multiple modes and the optimiser is getting stuck in different local minima. We have no way of determining whether a global minimum has been reached.

With Proc. A, bootstrapped $95 \%$ confidence intervals for the parameters (Table 4.2) and the predicted index (Figure 16) are much narrower than those for Proc. B ((Table 4.3 and Figure 18). Also, the univariate profile 
likelihood for $r$ and $K$ from Proc A. indicate similar narrow confidence intervals (Figure 17). For Proc. B we were only able to plot likelihood profile for parameter $r$ (Figure 19) and no meaningful plot could be generated for $K$.

Table 4.2: MLE boostrap estimates: Namibian hake, proc. A, opt im method L-BFSG-U

\begin{tabular}{rrrrrr}
\hline & & \multicolumn{3}{c}{ bootstrap median and 95\% C. I. } \\
\cline { 3 - 6 } & ML estimate & median & lower & upper & median bias \\
\hline$r$ & 0.38 & 0.38 & 0.29 & 0.46 & 0.60 \\
$K$ & 2737 & 2795 & 2406 & 3360 & 0.43 \\
$q$ & 0.00043 & 0.00042 & 0.00034 & 0.00051 & 0.54 \\
\hline
\end{tabular}

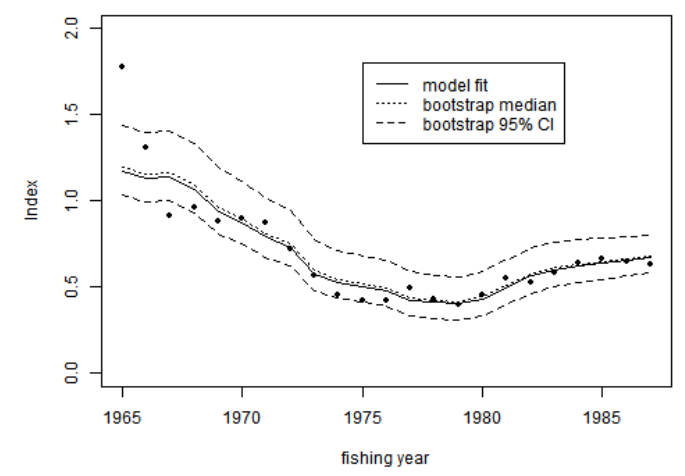

Figure 16: MLE: Namibian hake, proc. A, L-BFSG-U. Plot of observed index $\left(I_{t}=\bullet\right)$ and model predicted index $\left(\hat{I}_{t}\right)$ with its boostrapped median and $95 \%$ C.I.

(a)

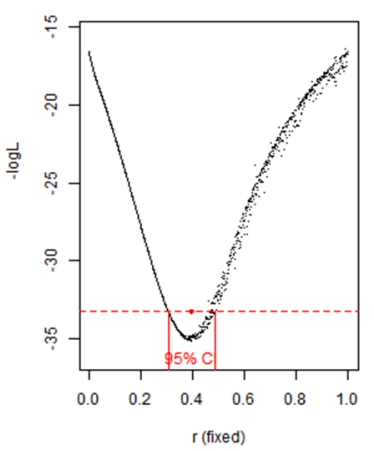

(b)

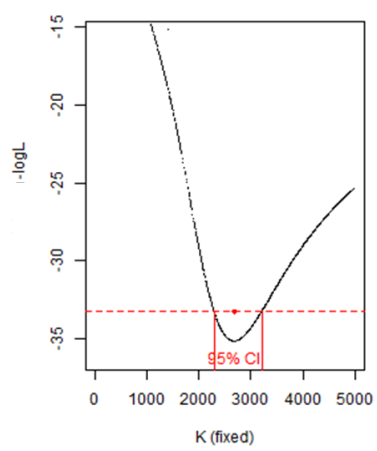

Figure 17: MLE plot of univariate likelihood profiles for (a) $r$ and (b) $K$ : Namibian hake, proc. A, optim method L-BFSG-U. 
Table 4.3: MLE boostrap estimates: Namibian hake, proc. B, opt im method SANN

\begin{tabular}{crrrrr}
\hline & & \multicolumn{4}{c}{ bootstrap median and 95\% C. I. } \\
\cline { 3 - 6 } & ML estimate & median & lower & upper & median bias \\
\hline$r$ & 0.37 & 0.03 & -6.30 & 0.37 & 0.98 \\
$K$ & 2779 & 16631 & 2851 & 177781 & 0.03 \\
$q$ & 0.00043 & 0.00012 & 0.00007 & 0.00043 & 0.98 \\
$B_{1}$ & 2726 & 7599 & 2448 & 12278 & 0.07 \\
\hline
\end{tabular}

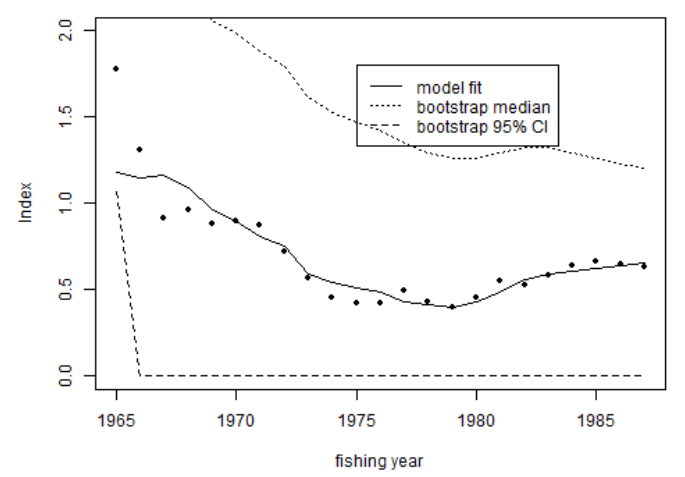

Figure 18: MLE plot of model fit: Namibian hake, Model B, SANN

(a)

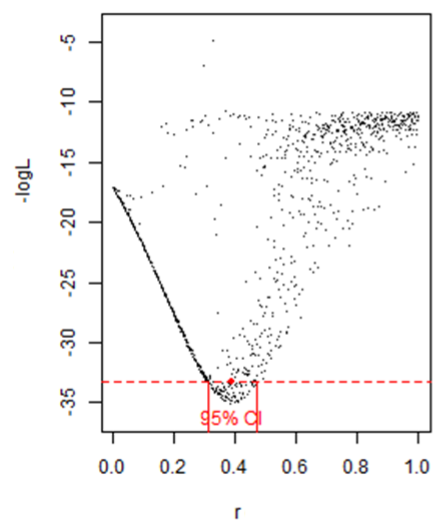

Figure 19: Plot of univariate likelihood profiles for $r$ : Namibian hake, Model B, SANN.

This MLE estimation method produced the best results with the Namibian hake dataset when $B_{1}$ was fixed $(=K)$, confirming recommendations in the literature [46]. By not including some sources of uncertainty (i.e. $B_{1}$ not included as an estimated parameter), the optimisation methods produced more consistent results that were less uncertain. This additional assumption needs to be taken into consideration when reporting results from the first procedure. 
Given that our datasets for the four rattail species may be less informative than the Namibian hake dataset (due to lack of contrast), this test indicates that the MLE method will not be successful in estimating $B_{1}$ for rattails.

\subsubsection{Rattail data}

For less than 2 units of difference (range: -10.136 to -8.523 ) in the minimum log-likelihood values between the two procedures for the rattail species Javelinfish, the two procedures produce very different parameter estimates (Table 4.4). For example, the estimated value of the growth rate parameter $\hat{r}$ varies widely, e.g. for $-\log L=-8.593, \hat{r}=2.2$ and for $-\log L=-10.136, \hat{r}=0.12$ (Table 4.4). Unlike the Namibian hake data, the parameter estimates for Javelinfish are unstable. This suggests that multimodality or flatness in the likelihood surface may be making it difficult for the optimisation methods to detect a global minimum.

Table 4.4:

Results of model fits for rattail species: Javelinfish (JAV)

\begin{tabular}{cccccccccr}
\hline $\begin{array}{c}\text { procedure } \\
\text { type }\end{array}$ & $\begin{array}{c}\text { optim } \\
\text { method }\end{array}$ & \multicolumn{2}{c}{ min. value } & \multicolumn{2}{c}{ parameter estimates: } & \multicolumn{3}{c}{ information criteria: } \\
\hline A & N-M & -8.52270 & 2.09 & 1362859 & 0.00822 & 0.4054 & & & \\
& L-BFG-U & -8.59311 & 2.20 & 5786765 & 0.00193 & 0.4052 & & -11.186 & -9.774 \\
& SANN & -8.56976 & 2.24 & 8128417 & 0.00137 & 0.4093 & & & \\
& MSB & -8.56463 & 2.21 & 9523320 & 0.00117 & 0.4081 & & & \\
\hline B & N-M & -9.30848 & 0.16 & 1414457 & 0.00847 & 0.1374 & 1157221 & & \\
& L-BFG-U & failed to converge & & & & & & \\
& SANN & -10.13569 & 0.12 & 8062731 & 0.00177 & 0.3566 & 4662709 & -12.271 & -9.771 \\
& MSB & -10.13082 & 0.13 & 2982900 & 0.00484 & 0.3751 & 1566629 & & \\
\hline
\end{tabular}

For Proc. A (withopt immethod L-BFGS-U) we ran bootstrapped 95\% confidence intervals for the parameters and predicted index, $\hat{I}_{t}$, as well as univariate likelihood profiles for $\hat{r}$ and $\hat{K}$. The likelihood profile curves (Figure 21) are flat, any curvature apparent in the plots is due to the greatly reduced scale of the profile likelihood value on the y-axis; the corresponding $95 \%$ confidence bounds encompass all the fixed parameter values for both $r$ and $K$. The predicted index (Figure 20) fits a line that runs through the middle of the observed index points and does not indicate any pattern of variation in the data. The $95 \%$ confidence interval for the index ranges from a lower bound of 0 , to an upper bound that is off the scale of the plot. 
Table 4.5: MLE boostrap estimates: Javelinfish, proc. A, (L-BFSG-U)

\begin{tabular}{rrrrrr}
\hline & & \multicolumn{3}{c}{ bootstrap median and 95\% C. I. } \\
\cline { 3 - 6 } & ML estimate & median & lower & upper & median bias \\
\hline$r$ & 2.20 & 0.55 & 0.10 & 2.65 & 0.91 \\
$K$ & 5786765 & 1574407 & 80353 & 52976118 & 0.73 \\
$q$ & 0.00193 & 0.00708 & 0.00022 & 0.17415 & 0.29 \\
\hline
\end{tabular}

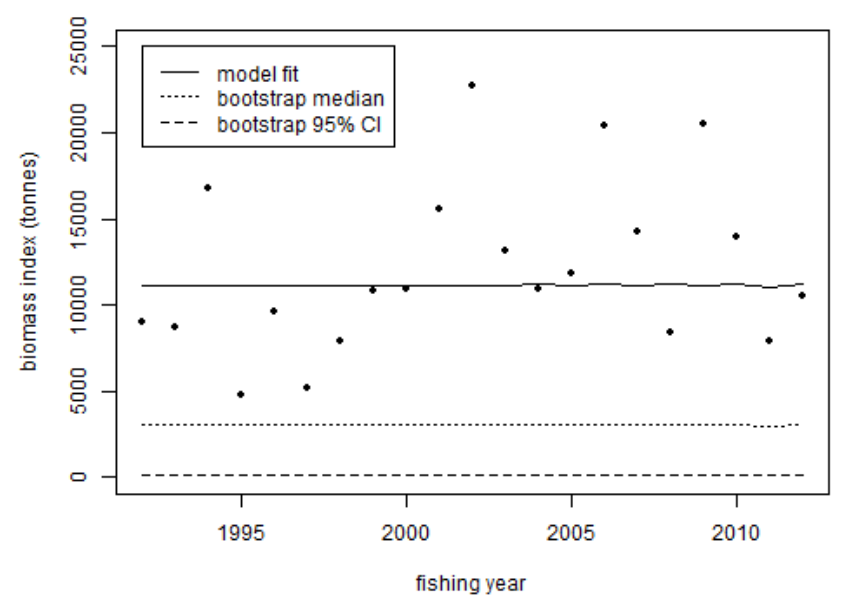

Figure 20: MLE: Javelinfish, Model A, L-BFSG-U. Plot of observed index $\left(I_{t}=\bullet\right)$ and model predicted index $\left(\hat{I}_{t}\right)$ with its bootstrapped median and 95\% C.I.

(a)

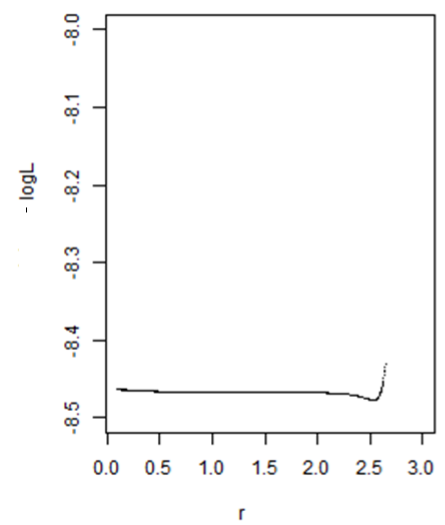

(b)

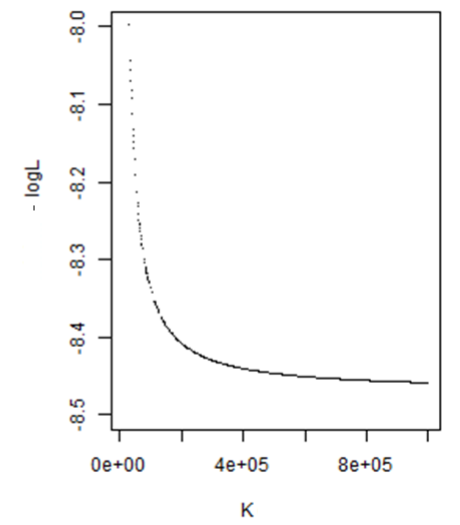

Figure 21: Plot of univariate likelihood profile for (a) $r$ and (b) $K$ : Javelinfish, Model A, L-BFSG-U. 
For the Javelinfish data the surplus production model produces a likelihood surface from which it is difficult to determine a global maximum (minimum negative log likelihood), and for all parameters estimated the uncertainty is high. Possible causes are a flat likelihood surface or a multimodal surface. This situation is not helped by the small sample size; 21 time points may not be enough to be highly informative about the parameters.

These characteristics of the Javelinfish results are similar for all the four rattail species analysed (MLE results for three other rattail species included in Appendix H). Clearly this method of inference is not suitable for these data. We apply a Bayesian estimation method to a reparameterisation of the surplus production model that allows for the incorporation of process and observation error. The Bayesian approach can help if the likelihood surface is flat (through the consolidation of an informative prior with the likelihood), and is useful for small sample sizes and poor data situations. 


\section{Chapter 5}

\section{Bayesian estimation of state-space model parameters}

Bayesian estimation provides a mechanism (not available with MLE) to incorporate additional information about model parameters, in the form of a prior probability distribution. A prior distribution is a formulaic representation of knowledge about the parameters that is available prior to data collection. Consolidation of this prior information with that available in the data (likelihood function) results in a posterior distribution of the parameters. All inference about the parameters is based on this distribution. this may result in improved parameter estimation since incorporation of additional prior information may reduce the uncertainty associated with parameters. This would in turn give a better fit between the observed and the predicted indices.

The prior distribution is determined subjectively, mainly from expert opinion or previous studies, and the amount of information contained in the prior (compared to the data) will determine which is more dominant in determining posterior parameter distributions [21]. As such, the definition of prior information assigned to each parameter is critical to the model outcomes, and it is important to test the sensitivity of resulting parameter estimates to changes in the specification of priors.

A further development in this chapter is the use of a state-space surplus production model for the rattail species data to incorporate uncertainty in both the observation and process models. We follow the parameterisation and Bayesian approach as applied by Millar and Meyer [66], and implement this estimation method using WinBUGS [55] in R (R2WinBUGS [90]). 


\subsection{Bayesian estimation}

For a given model, let $\theta$ represent the parameter vector and $y$ represent the data. Estimates of the model parameters, $\theta$, can be obtained from a posterior distribution conditional on the observed data, $y$. To apply the Bayesian method we: 1) decide on the prior distributions, 2) decide on the likelihood, 3) apply Bayes' rule to derive the posterior distribution, and 4) derive inference statistics from the posterior, with all unknown quantities treated as random variables.

The posterior distribution $\pi(\theta \mid y)$ allows us to make probability statements about the parameters given the data and is based on their joint probability distribution $\pi(\theta, y)$ [39]. The posterior distribution given by

$$
\begin{aligned}
\pi(\theta \mid y) & =\frac{f(y \mid \theta) \pi(\theta)}{f(y)} \\
& =\frac{f(y \mid \theta) \pi(\theta)}{\int \pi(y, \theta) \pi(\theta) d \theta},
\end{aligned}
$$

where $\pi(\theta)$ is the prior distribution, and $f(y \mid \theta)$ the likelihood function.

The denominator $\int \pi(y, \theta) \pi(\theta) d \theta=f(y)$ is the marginal distribution of $y$ and is a normalising constant for the posterior distribution. For estimation purposes, the posterior only needs to be known to a constant of proportionality [39], and we now have

$$
\begin{aligned}
\pi(\theta \mid y) & =\frac{1}{f(y)} f(y \mid \theta) \pi(\theta) \\
& \propto \ell(\theta \mid y) \pi(\theta)
\end{aligned}
$$

Point and interval estimates of the parameters can be obtained from summary statistics of the posterior distribution. These estimates can be obtained analytically for posterior distributions with integrals that have closed form solutions. Where this is not possible Monte Carlo integration can be used to evaluate intractable integrals by approximating expectations using randomly sampled values from the posterior distribution. When it is not possible to draw independent samples from the posterior distribution Markov chain Monte Carlo (MCMC) methods can be applied, where the independence assumption is relaxed and a large collection of dependent samples is constructed. Dependent samples are obtained by constructing a Markov chain whose stationary distribution is the posterior distribution The related draws are then used to obtain Monte Carlo type estimates. Classical MCMC methods such as the Metropolis-Hastings algorithm [63,43] and Gibbs sampler [38] are general purpose sampling tools 
and have become more widely used in statistics since the 1990's. Such methods are prominent tools in Bayesian analysis.

\subsection{State-space surplus production model}

State space models relate the time series observations $I_{t}$ to unobserved "states" $B_{t}$ through a stochastic observation model for $I_{t}$ given by

$$
I_{t}=q B_{t} e^{\varepsilon_{t}^{u}}
$$

The states are assumed to follow a stochastic transition model of surplus production [64]

$$
B_{t}=\left(B_{t-1}+r B_{t-1}\left(1-\frac{B_{t-1}}{K}\right)-C_{t-1}\right) e^{\varepsilon_{t}^{v}}
$$

where (as before)

$$
\varepsilon_{t}^{u} \stackrel{\mathrm{iid}}{\sim} N\left(0, \sigma_{u}^{2}\right), \quad \varepsilon_{t}^{v} \stackrel{\mathrm{iid}}{\sim} N\left(0, \sigma_{v}^{2}\right)
$$

Due to known computational difficulties with the parameterisation in eq. (5.4) leading to poor performance in the Metropolis-Hastings within-Gibbs sampler, Miller and Meyer reparameterised the model by replacing the states $B_{t}$ with $P_{t}=B_{t} / K$. The new states are the ratio of biomass to carrying capacity whereby $B_{t}$ is replaced by $K P_{t}$, and the reformulated model equations are

$$
\begin{aligned}
\log \left(P_{1}\right) & \sim N\left(0, \sigma_{v}^{2}\right) \\
P_{t} & =\left(P_{t-1}+r P_{t-1}\left(1-P_{t-1}\right)-\frac{C_{t-1}}{K}\right) e^{\varepsilon_{t}^{v}} \quad t=2, \ldots, n \\
I_{t} & =Q P_{t} e^{\varepsilon_{t}^{u}}
\end{aligned}
$$

where $Q=q K$.

The error structure includes both $\varepsilon_{t}^{u}$ and $\varepsilon_{t}^{v}$, which are described as before by eq. (3.14) on page 32 and eq. (3.20) on page 33.

The probability density function $f(\cdot)$ for $P_{t}$ and $I_{t}$ are that of a $\log$ Normal random variable

$$
\begin{aligned}
f\left(P_{1} \mid \sigma_{v}^{2}\right) & =\frac{1}{P_{1} \sqrt{2 \pi \sigma_{v}^{2}}} \exp \left(-\frac{1}{2 \sigma_{v}^{2}}\left[\log \left(P_{1}\right)\right]^{2}\right) \\
f\left(P_{t} \mid r, K, P_{t-1}, \sigma_{v}^{2}\right) & =\frac{1}{P_{t} \sqrt{2 \pi \sigma_{v}^{2}}} \exp \left(-\frac{1}{2 \sigma_{v}^{2}}\left[\log \left(\frac{P_{t}}{P_{t-1}+r P_{t-1}\left(1-P_{t-1}\right)-\frac{C_{t-1}}{K}}\right)\right]^{2}\right) \\
f\left(I_{t} \mid r, k, q, P_{t}, \sigma_{u}^{2}\right) & =\frac{1}{I_{t} \sqrt{2 \pi \sigma_{u}^{2}}} \exp \left(-\frac{1}{2 \sigma_{u}^{2}}\left[\log \left(I_{t}\right)-\log \left(Q P_{t}\right)\right]^{2}\right)
\end{aligned}
$$


and, therefore

$$
\begin{aligned}
\log \left(P_{1}\right) & \sim N\left(0, \sigma_{v}^{2}\right) \\
\log \left(P_{t}\right) & \sim N\left(\log \left[P_{t-1}+r P_{t-1}\left(1-P_{t-1}\right)-\frac{C_{t-1}}{K}\right], \sigma_{v}^{2}\right) \quad t=2, \ldots, n(5.1 \\
\log \left(I_{t}\right) & \sim N\left(\log \left[Q P_{t}\right], \sigma_{u}^{2}\right)
\end{aligned}
$$

The posterior distribution of the unknowns - the model parameters and surplus production "states" $\boldsymbol{\theta}=\left(r, K, q, \sigma_{u}, \sigma_{v}, \boldsymbol{P}\right)$, given the data $\boldsymbol{I}$ (and fixed catches $C$ ) - is proportional to the product of the prior and the data likelihood

$$
\pi(\boldsymbol{\theta} \mid \boldsymbol{I}, \boldsymbol{C}) \propto \pi(\boldsymbol{\theta} \mid \boldsymbol{C}) L(\boldsymbol{\theta} \mid \boldsymbol{I}, \boldsymbol{C})
$$

where, assuming prior independence between $r, K, q, \sigma_{u}$ and $\sigma_{v}$, and assuming conditional independence between subsequent states,

$$
\begin{aligned}
\pi(\boldsymbol{\theta} \mid \boldsymbol{C}) & =\pi\left(r, K, q, \sigma_{u}, \sigma_{v}, \boldsymbol{P} \mid \boldsymbol{C}\right) \\
& =\pi(r) \pi(K) \pi(q) \pi\left(\sigma_{u}\right) \pi\left(\sigma_{v}\right) \pi\left(\boldsymbol{P} \mid r, K, \sigma_{v}, \boldsymbol{C}\right) \\
& =\pi(r) \pi(K) \pi(q) \pi\left(\sigma_{u}\right) \pi\left(\sigma_{v}\right) \pi\left(P_{1} \mid \sigma_{v}\right) \prod_{t=2}^{n} \pi\left(P_{t} \mid P_{t-1}, r, K, \sigma_{v}, C_{t-1}\right)
\end{aligned}
$$

and

$$
\begin{aligned}
L(\boldsymbol{\theta} \mid \boldsymbol{I}, \boldsymbol{C}) & =f\left(I_{t} \mid q, K, \sigma_{u}, \boldsymbol{P}\right) \\
& =\prod_{t=1}^{n} f\left(I_{t} \mid q, K, \sigma_{u}, P_{t}\right)
\end{aligned}
$$

The likelihood contains all the information about parameters and states that comes directly from the data, and the joint prior density $\pi\left(r, K, q, \sigma_{u}, \sigma_{v}, \boldsymbol{P}\right)$ contains all the prior information about the parameters and the states.

The priors represent knowledge (which may, or may not, be informative) about the parameters before any data are observed. In their discussion on prior selection in fisheries models, Punt and Hilborn [79] note that "wellthought-out informative priors can reduce uncertainty considerably" and "using noninformative priors implies that no information from fisheries science is relevant to the parameter in question". They also recommend that in the absence of information on which to base a prior it would be better to select a noninformative prior and then test sensitivity to alternatives, rather than specify an informative prior based on speculation. 


\subsection{Specification of prior distributions}

\section{Prior for intrinsic growth rate, $r$}

The prior distribution for the intrinsic growth rate $\pi(r)$ was set as a lognormal distribution with parameters $\mu_{r}$ and $\sigma_{r}^{2}$,

$$
\pi(r)=\frac{1}{r \sqrt{2 \pi \sigma_{r}^{2}}} e^{-\left(\log (r)-\mu_{r}\right)^{2} / 2 \sigma_{r}^{2}}, r>0
$$

The lognormal distribution was used since only positive values of $r$ are possible.To determine the parameter values for $\mu_{r}$ and $\sigma_{r}^{2}$ we set

$$
\begin{aligned}
a & =E(r)=e^{\mu_{r}+\frac{1}{2} \sigma_{r}^{2}} \\
b & =\operatorname{Var}(r)=e^{2 \mu_{r}+\sigma_{r}^{2}}\left(e^{\sigma_{r}^{2}}-1\right)
\end{aligned}
$$

where values of $a$ and $b$ are determined from fisheries science research and elicted expert knowledge. We then have

$$
\begin{aligned}
a & =e^{\mu_{r}+\frac{1}{2} \sigma_{r}^{2}} \\
b & =e^{2 \mu_{r}+\sigma_{r}^{2}}\left(e^{\sigma_{r}^{2}}-1\right) \\
& =a^{2}\left(e^{\sigma_{r}^{2}}-1\right) \\
\therefore e^{\sigma_{r}^{2}}-1 & =\frac{b}{a^{2}} \\
e^{\sigma_{r}^{2}} & =\frac{b}{a^{2}}+1 \\
\sigma_{r}^{2} & =\log \left(\frac{b}{a^{2}}+1\right)
\end{aligned}
$$

and from eq. (5.19)

$$
\begin{aligned}
\mu_{r}+\frac{1}{2} \sigma_{r}^{2} & =\log (a) \\
\mu_{r} & =\log (a)-\frac{1}{2} \log \left(\frac{b}{a^{2}}+1\right) \quad \text { using eq. }(5.21)
\end{aligned}
$$

Therefore, for given values for $a=E(r)$ and $b=E(r)$ we use eqn.s (5.21) and (5.22) to determine parameter values for the lognormal distribution.

Meta-analysis of the intrinsic growth rate from studies of 46 stocks from the order (Gadiformes), of which the rattail family (Macrouridae) also belong to, list a range for $r$ from 0.17 to 0.96 , with a mean of 0.47 and median of 0.45, (P. Neubauer, Dragonfly, personal communication, 29 August 
2013). An expert opinion from fisheries science expected the mean growth rate for rattail stocks to be around 0.4 (with s.e. of 0.25 ), considering rattail stocks would have a growth rate somewhere between unproductive species with a rate close to 0.1 (e.g. sharks) and productive stocks that have a growth rate at or above 1 (M.Dunn, VUW, personal communication, 2013).

\section{Prior for carrying capacity, $K$}

We have no expert or fisheries science information on which to base an informative prior for $K$. An uninformed prior for carrying capacity can be based on a uniform distribution over a large range of values to enable equal credibility for small and large possible values of $K$ [59]. To avoid implausibly large posterior expected values for $K$ that might arise when there is little information in the data about $K$, McAllister and Kirkwood [60] recommend setting a Uniform distribution for $\log (K)$, which assigns lower credibility to higher values for $K$.

Making use of the reparameterisation $k=1 / K[64,66]$, we construct a lognormal prior for $k$, with 10th and 90th percentile points of this distribution based on the lower and upper limits from the Uniform distribution on $\log (K)$. That is, we have

$$
\begin{aligned}
\log (K) & \sim U(\log (a), \log (b)) \\
k & \sim \log N\left(\mu_{k}, \sigma_{k}^{2}\right)
\end{aligned}
$$

From

$$
P\left(Z<\frac{\log (a)-\mu_{k}}{\sigma_{k}}\right)=0.1 \quad P\left(Z>\frac{\log (b)-\mu_{k}}{\sigma_{k}}\right)=0.1
$$

where $Z_{\alpha=0.1}= \pm 1.281552$, we have

$$
\begin{aligned}
\sigma_{k} & =\log \left(\frac{a}{b}\right) 2(1.281552) \\
\mu_{k} & =\log \left(\frac{1}{a}\right)+1.281552\left(\sigma_{k}\right)
\end{aligned}
$$

We set the value of $a$ as the maximum catch in the time series (e.g. 6,500 tonnes for Javelinfish). The upper limit $(b)$ needed to be set at a high enough level to reflect the magnitude of exploitable biomass [18], and this value was set arbitrarily at 20 times the maximum catch rate, (e.g. 130,000 tonnes for Javelinfish).

Therefore, for given values of $a=\max \left(C_{1}, \ldots, C_{n}\right)$ and an arbitrarily chosen $b$, we determine values of $\sigma_{k}^{2}$ and $\mu_{k}$ using equations (5.26 and 5.27). 


\section{Prior for catchability, $q$}

We have no information on which to develop an informative prior for the catchability coefficient. We set a non-informative prior by assigning an inverse-gamma distribution [64]

$$
\pi(q)=\frac{\beta^{\alpha}}{\Gamma(\alpha)} q^{-(\alpha+1)} \exp \left(\frac{-\beta}{q}\right)
$$

with scale and shape parameters $\beta=\alpha=10^{3}$.

Priors for the error variances, $\sigma_{u}^{2}$ and $\sigma_{v}^{2}$

We also set non-informative priors for the variances of the observation error $\sigma_{u}$ and the process error $\sigma_{v}$ based on the inverse gamma distribution

$$
\begin{aligned}
& \pi\left(\sigma_{u}^{2}\right)=\frac{\delta_{u}^{\gamma_{u}}}{\Gamma\left(\gamma_{u}\right)}\left(\sigma_{u}^{2}\right)^{-\left(\gamma_{u}+1\right)} \exp \left(\frac{-\delta_{u}}{\sigma_{u}^{2}}\right) \\
& \pi\left(\sigma_{u}^{2}\right)=\frac{\delta_{v}^{\gamma_{v}}}{\Gamma\left(\gamma_{v}\right)}\left(\sigma_{v}^{2}\right)^{-\left(\gamma_{v}+1\right)} \exp \left(\frac{-\delta_{v}}{\sigma_{v}^{2}}\right)
\end{aligned}
$$

with the scale and shape parameters set to $\delta_{u}=\gamma_{u}=10^{3}$ and $\delta_{v}=\gamma_{v}=10^{3}$.

\subsection{Sampling from the posterior distribution}

The joint posterior distribution is

$$
\begin{aligned}
& \pi\left(r, K, q, \sigma_{u}, \sigma_{v}, \boldsymbol{P} \mid \boldsymbol{I}, \boldsymbol{C}\right) \\
& \propto \pi(r) \pi(K) \pi(q) \pi\left(\sigma_{u}\right) \pi\left(\sigma_{v}\right) \pi\left(P_{1} \mid \sigma_{v}\right) \prod_{t=2}^{n} \pi\left(P_{t} \mid r, K, P_{t-1}, \sigma_{v}\right) \prod_{t=1}^{n} f\left(I_{t} \mid K, q, P_{t}, \sigma_{u}\right) \\
&= \frac{1}{r \sqrt{2 \pi} \sigma_{r}} \exp \left(-\frac{\left(\log r-\mu_{r}\right)^{2}}{2 \sigma_{r}^{2}}\right) \times \frac{1}{(b-a)} \times \frac{\beta^{\alpha}}{\Gamma(\alpha)} q^{-(\alpha+1)} \exp \left(\frac{-\beta}{q}\right) \\
& \times \frac{\delta_{u}^{\gamma_{u}}}{\Gamma\left(\gamma_{u}\right)}\left(\sigma_{u}^{2}\right)^{-\left(\gamma_{u}+1\right)} \exp \left(\frac{-\delta_{u}}{\sigma_{u}^{2}}\right) \times \frac{\delta_{v}^{\gamma_{v}}}{\Gamma\left(\gamma_{v}\right)}\left(\sigma_{v}^{2}\right)^{-\left(\gamma_{v}+1\right)} \exp \left(\frac{-\delta_{v}}{\sigma_{v}^{2}}\right) \\
& \times \frac{1}{P_{1} \sqrt{2 \pi \sigma_{v}^{2}}} \exp \left(-\frac{1}{2 \sigma_{v}^{2}}\left[\log \left(P_{1}\right)\right]^{2}\right) \\
& \times \prod_{t=2}^{n} \frac{1}{P_{t} \sqrt{2 \pi \sigma_{v}^{2}}} \exp \left(-\frac{1}{2 \sigma_{v}^{2}}\left[\log \left(\frac{P_{t}}{P_{t-1}+r P_{t-1}\left(1-P_{t-1}\right)-\frac{C_{t-1}}{K}}\right)\right]^{2}\right) \\
& \times \prod_{t=1}^{n} \frac{1}{I_{t} \sqrt{2 \pi \sigma_{u}^{2}}} \exp \left(-\frac{1}{2 \sigma_{u}^{2}}\left[\log \left(\frac{I_{t}}{Q P_{t}}\right)\right]^{2}\right)
\end{aligned}
$$


To estimate the parameters of this non-linear, multiparameter model we generate a large number of samples from the posterior distribution using Markov Chain Monte Carlo (MCMC) simulation. We use Gibbs sampling (adapting code from Millar and Meyer [65]) which divides the posterior distribution into components that can be updated one at a time, making the computational process more tractable [55].

For our vector of parameters and states $\boldsymbol{\theta}=\left(r, K, q, \sigma_{u}, \sigma_{v}, P_{1}, P_{2}, \ldots, P_{21}\right)$, the Gibbs sampler iteratively samples from the conditional distribution of each parameter given the current values of all the other parameters in the model (instead of updating $\boldsymbol{\theta}$ as a block). The full conditional distributions are only needed up to a proportionality constant as they are proportional to $\pi(\boldsymbol{\theta}) \times L(\boldsymbol{\theta} \mid \boldsymbol{I}, \boldsymbol{C})$.

The Gibbs sampler algorithm for our posterior distribution can be summarised as

Let $\boldsymbol{\theta}^{(j)}=\left(r^{(j)}, K^{(j)}, q^{(j)}, \sigma_{u}^{(j)}, \sigma_{v}^{(j)}, P_{1}^{(j)}, P_{2}^{(j)}, \ldots, P_{21}^{(j)}\right)$ be the $j^{\text {th }}$ draw of our parameter vector $\boldsymbol{\theta}$.

Draw a new vector $\boldsymbol{\theta}^{(j+1)}$ from the following distributions:

$$
\begin{aligned}
r^{(j+1)} & \sim \pi\left(r \mid K^{(j)}, q^{(j)}, \sigma_{u}^{(j)}, \sigma_{v}^{(j)}, P_{1}^{(j)}, P_{2}^{(j)}, \ldots, P_{21}^{(t)}, \boldsymbol{I}, \boldsymbol{C}\right) \\
K^{(j+1)} & \sim \pi\left(K \mid r^{(j+1)}, q^{(j)}, \sigma_{u}^{(j)}, \sigma_{v}^{(j)}, P_{1}^{(j)}, P_{2}^{(j)}, \ldots, P_{21}^{(j)}, \boldsymbol{I}, \boldsymbol{C}\right) \\
q^{(j+1)} \sim & \pi\left(q \mid r^{(j+1)}, K^{(j+1)}, \sigma_{u}^{(j)}, \sigma_{v}^{(j)}, P_{1}^{(j)}, P_{2}^{(j)}, \ldots, P_{21}^{(j)}, \boldsymbol{I}, \boldsymbol{C}\right) \\
\sigma_{u}^{(j+1)} \sim & \pi\left(\sigma_{u} \mid r^{(j+1)}, K^{(j+1)}, q^{(j+1)}, \sigma_{v}^{(j)}, P_{1}^{(j)}, P_{2}^{(j)}, \ldots, P_{21}^{(j)}, \boldsymbol{I}, \boldsymbol{C}\right) \\
\sigma_{v}^{(j+1)} & \sim \pi\left(\sigma_{v} \mid r^{(j+1)}, K^{(j+1)}, q^{(j+1)}, \sigma_{u}^{(j+1)}, P_{1}^{(j)}, P_{2}^{(j)}, \ldots, P_{21}^{(j)}, \boldsymbol{I}, \boldsymbol{C}\right) \\
P_{1}^{(j+1)} & \sim \pi\left(P_{1} \mid r^{(j+1)}, K^{(j+1)}, q^{(j+1)}, \sigma_{u}^{(j+1)}, \sigma_{v}^{(j+1)}, P_{2}, P_{3}^{(j)}, \ldots, P_{21}^{(j)}, \boldsymbol{I}, \boldsymbol{C}\right) \\
P_{2}^{(j+1)} & \sim \pi\left(P_{2} \mid r^{(j+1)}, K^{(j+1)}, q^{(j+1)}, \sigma_{u}^{(j+1)}, \sigma_{v}^{(j+1)}, P_{1}^{(j+1)}, P_{3}^{(j)}, \ldots, P_{21}^{(j)}, \boldsymbol{I}, \boldsymbol{C}\right) \\
\vdots & \vdots \\
P_{21}^{(j+1)} & \sim \pi\left(P_{21} \mid r^{(j+1)}, K^{(j+1)}, q^{(j+1)}, \sigma_{u}^{(j+1)}, \sigma_{v}^{(j+1)}, P_{1}^{(j+1)}, P_{2}^{(j+1)}, \ldots, P_{20}^{(j+1)}, \boldsymbol{I}, \boldsymbol{C}\right)
\end{aligned}
$$

Repeat $M$ times to get $M$ draws of each parameter and state from the posterior (assuming convergence). 
The full conditional posterior distributions are defined as

$$
\begin{aligned}
& \pi\left(r \mid K, q, \sigma_{u}, \sigma_{v}, \boldsymbol{P}, \boldsymbol{I}, \boldsymbol{C}\right) \propto \exp \left(-\frac{\log \left(r-\mu_{r}\right)^{2}}{2 \sigma_{r}^{2}}\right) \prod_{t=2}^{n} f\left(P_{t} \mid r, K, P_{t-1}, \sigma_{v}, C_{t-1}\right) \\
& \times f\left(P_{1} \mid \sigma_{v}\right) \prod_{t=1}^{n} f\left(I_{t} \mid K, q, P_{t}, \sigma_{u}\right) \\
& \pi\left(K \mid r, q, \sigma_{u}, \sigma_{v}, \boldsymbol{P}, \boldsymbol{I}, \boldsymbol{C}\right) \propto \frac{1}{b-a} \prod_{t=2}^{n} f\left(P_{t} \mid r, K, P_{t-1}, \sigma_{v}, C_{t-1}\right) \\
& \times f\left(P_{1} \mid \sigma_{v}\right) \prod_{t=1}^{n} f\left(I_{t} \mid K, q, P_{t}, \sigma_{u}\right) \\
& \pi\left(q \mid K, \sigma_{u}, \sigma_{v}, \boldsymbol{P}, \boldsymbol{I}\right) \propto q^{-\alpha-1} \exp \left(\frac{-\beta}{q}\right) \\
& \times \prod_{t=1}^{n} f\left(I_{t} \mid K, q, P_{t}, \sigma_{u}\right) \\
& \pi\left(\sigma_{u} \mid K, q, \sigma_{v}, \boldsymbol{P}, \boldsymbol{I}\right) \propto\left(\sigma_{u}^{2}\right)^{-\gamma_{u}-1} \exp \left(\frac{-\delta_{u}}{\sigma_{u}^{2}}\right) \\
& \times \prod_{t=1}^{n} f\left(I_{t} \mid K, q, P_{t}, \sigma_{u}\right) \\
& \pi\left(\sigma_{v} \mid r, K, q, \sigma_{u}, \boldsymbol{P}, \boldsymbol{I}, \boldsymbol{C}\right) \propto\left(\sigma_{v}^{2}\right)^{-\gamma_{v}-1} \exp \left(\frac{-\delta_{v}}{\sigma_{v}^{2}}\right) f\left(P_{1} \mid \sigma_{v}\right) \\
& \times \prod_{t=2}^{n} f\left(P_{t} \mid r, K, P_{t-1}, \sigma_{v}, C_{t-1}\right)
\end{aligned}
$$

for $t=1$

$$
\begin{aligned}
\pi\left(P_{1} \mid r, K, q, \sigma_{u}, \sigma_{v}, P_{2}, P_{3}, \ldots, P_{n}, \boldsymbol{I}\right) \propto & f\left(P_{1} \mid \sigma_{v}\right) \\
& \times f\left(I_{1} \mid K, q, P_{1}, \sigma_{u}\right)
\end{aligned}
$$

for $t=2, \ldots, n$

$\pi\left(P_{t} \mid r, K, q, \sigma_{u}, \sigma_{v}, P_{1}, \ldots, P_{t-1}, P_{t+1}, \ldots, P_{n}, \boldsymbol{I}, \boldsymbol{C}\right) \propto \times f\left(P_{t} \mid r, K, P_{t-1}, \sigma_{v}, C_{t-1}\right)$

$\times f\left(I_{t} \mid K, q, P_{t}, \sigma_{u}\right)$ 
The MCMC algorithm in the Gibbs sampler produces a chain of simulated draws from a distribution where each draw is dependent on the previous draw. We have approximate draws from the posterior distribution when the chain converges to a stationary distribution. While the outputs from MCMC are correlated samples not iid samples, inference from the correlated samples is valid due to the ergodic theorem for Markov Chains, i.e. given samples $\boldsymbol{\theta}_{1}, \ldots, \boldsymbol{\theta}_{M}$ and any function $g(\boldsymbol{\theta})$ of the parameters, then

$$
\lim _{M \rightarrow \infty} \frac{1}{M} \sum_{m=1}^{M} g\left(\boldsymbol{\theta}_{m}\right)=E_{\boldsymbol{\theta} \mid \boldsymbol{y}}[g(\boldsymbol{\theta})]
$$

Sampling from the posterior distribution for each of the four rattail species was conducted in the same manner. We used WinBUGS to implement the MCMC simulation, in particular calling the OpenBUGS version from R. Processes within WingBUGS determine the most approriate sampling scheme for each node (unknown parameter), ranging from direct sampling using standard algorithms for full conditionals in closed form, to Metropolis-Hastings sampling when the full conditional is not in closed form [55]. The WinBUGS code for the model (adapted from Miller and Meyer [65]) is shown in Appendix I.

We ran 5 chains from diverse initial values (Table 5.1), each of 5 million samples, discarding the first 1 million samples (burn in) to remove dependence on the initial conditions, and thinning the remaining sample in each chain by 500 to reduce autocorrelations. The resulting 40,000 samples were examined for mixing properties (traceplots, density plots, autocorrelations, rejection rates) and tested for convergence using the diagnostic tools available in R's coda package [74]. Key model parameters and the model states $\left(r, K, q, \sigma_{u}^{2}, \sigma_{v}^{2}, P_{t}\right)$ were monitored and convergence checked using the Gelman and Rubin diagnostic (potential scale reduction factor) [40] and the Heidelberger and Welch diagnostic (stationarity test) [44].

Table 5.1: Convergence checks: initial starting values for parameters in 5 chains

\begin{tabular}{lccccc}
\hline & $r$ & $1 / K$ & $1 / q$ & $1 / \sigma_{u}^{2}$ & $1 / \sigma_{v}^{2}$ \\
\hline chain 1 & 0.1 & 0.005 & 100 & 100 & 100 \\
chain 2 & 0.3 & 0.004 & 125 & 125 & 125 \\
chain 3 & 0.5 & 0.003 & 150 & 150 & 150 \\
chain 4 & 0.7 & 0.002 & 175 & 175 & 175 \\
chain 5 & 0.9 & 0.001 & 200 & 200 & 200 \\
\hline
\end{tabular}


Visually, we checked trace plots (plot of parameter value at each iteration) for indications that the chain was not stuck in the parameter space (mixing poorly) and density plots for indications that the posterior was unimodal. There was no evidence in the trace plots that the chain remained in one area for extended periods as shown in the plots included for parameters $r, K$ and $q$ for Javelinfish(Figures 22-24). Plots for the three other rattail species are included in Appendix J (Figures 37-45).

We also checked for high rejection rates as an indication of a poorly mixing chain (a "rejection" has occurred if the value of a sample is the same at two successive iterations [74]). The acceptance rates for parameters $r, K$, $q, \sigma^{2}, \sigma_{v}^{2}$ are all over $99.9 \%$ (Table 5.2), except for $K$ and $q$ for Javelinfish and $K$ for Oblique banded rattail, where the acceptance rate is slightly less.

Table 5.2: Results: posterior distribution convergence diagnostic tests for multiple chains

\begin{tabular}{|c|c|c|c|}
\hline $\begin{array}{l}\text { Rattail species } \\
\text { and parameter }\end{array}$ & $\begin{array}{c}\text { acceptance rate } \\
(\%)\end{array}$ & $\begin{array}{l}\text { Gelman/Rubin diagnostic } \\
\text { Scale reduction factor } \hat{R} \\
\text { point estimate (upper C. I.) }\end{array}$ & $\begin{array}{l}\text { Heidelberger/Welch diagnostic } \\
\text { Stationarity test }\end{array}$ \\
\hline \multicolumn{4}{|l|}{ Javelinfish (JAV) } \\
\hline $\mathrm{r}$ & 99.99 & $1(1)$ & all chains passed \\
\hline K & 99.73 & $1.02(1.02)$ & 1 chain failed (\#5) \\
\hline$q$ & 99.71 & $1(1)$ & all chains passed \\
\hline$\sigma_{u}^{\frac{1}{2}}$ & 99.96 & $1(1)$ & all chains passed \\
\hline$\sigma_{v}^{u}$ & 99.99 & $1(1)$ & all chains passed \\
\hline \multicolumn{4}{|l|}{ Bollon's rattail (CBO) } \\
\hline $\mathrm{r}$ & 99.99 & $1(1)$ & all chains passed \\
\hline K & 99.96 & $1.02(1.02)$ & all chains passed \\
\hline$q$ & 99.95 & $1(1)$ & all chains passed \\
\hline$\sigma_{u}^{2}$ & 99.95 & $1(1)$ & all chains passed \\
\hline$\sigma_{v}^{a}$ & 99.99 & $1(1)$ & all chains passed \\
\hline \multicolumn{4}{|l|}{ Oliver's rattail (COL) } \\
\hline $\mathrm{r}$ & 99.99 & $1.05(1.06)$ & all chains passed \\
\hline K & 99.97 & $1(1)$ & all chains passed \\
\hline $\mathrm{q}$ & 99.97 & $1(1)$ & all chains passed \\
\hline$\sigma_{u}^{2}$ & 99.97 & $1(1)$ & all chains passed \\
\hline$\sigma_{v}^{a}$ & 99.98 & $1.01(1.01)$ & all chains passed \\
\hline \multicolumn{4}{|l|}{ Oblique banded rattail (CAS) } \\
\hline $\mathrm{r}$ & 99.97 & $1(1)$ & all chains passed \\
\hline K & 99.70 & $1.19(1.25)$ & 1 chain failed (\#5) \\
\hline$q$ & 99.92 & $1(1)$ & all chains passed \\
\hline$\sigma_{u}^{\frac{1}{2}}$ & 99.97 & $1(1)$ & all chains passed \\
\hline$\sigma_{v}^{a}$ & 99.99 & $1(1)$ & all chains passed \\
\hline
\end{tabular}



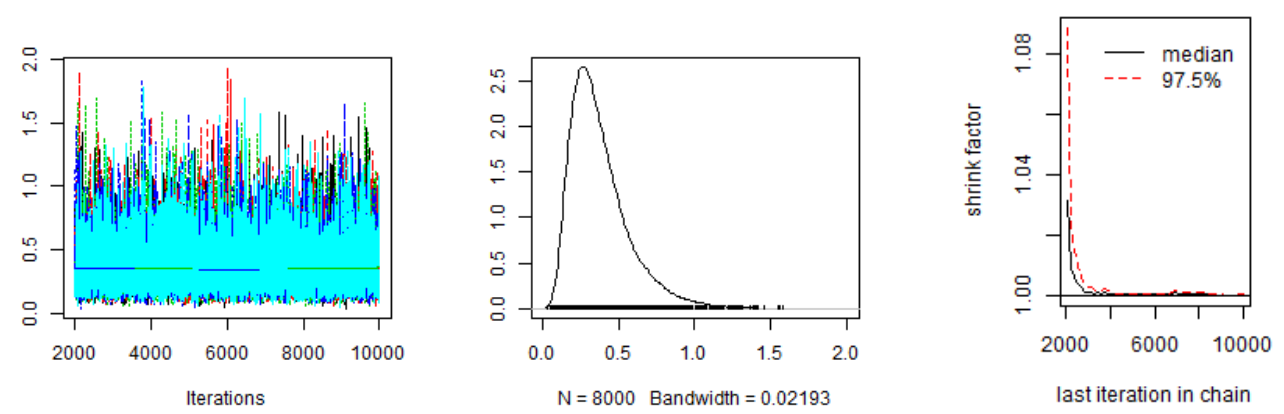

(a) Trace plot, density plot and Gelman-Rubin diagnostic plot.

Figure 22: Javelinfish (JAV): Convergence checks of posterior sample of $r$.
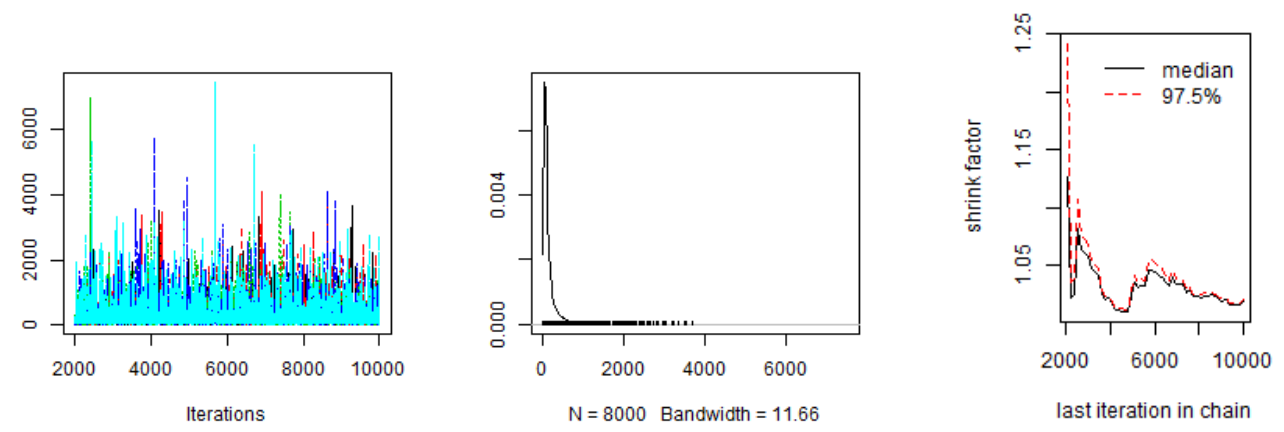

(a) Trace plot, density plot and Gelman-Rubin diagnostic plot.

Figure 23: Javelinfish (JAV): Convergence checks of posterior sample of $K$.
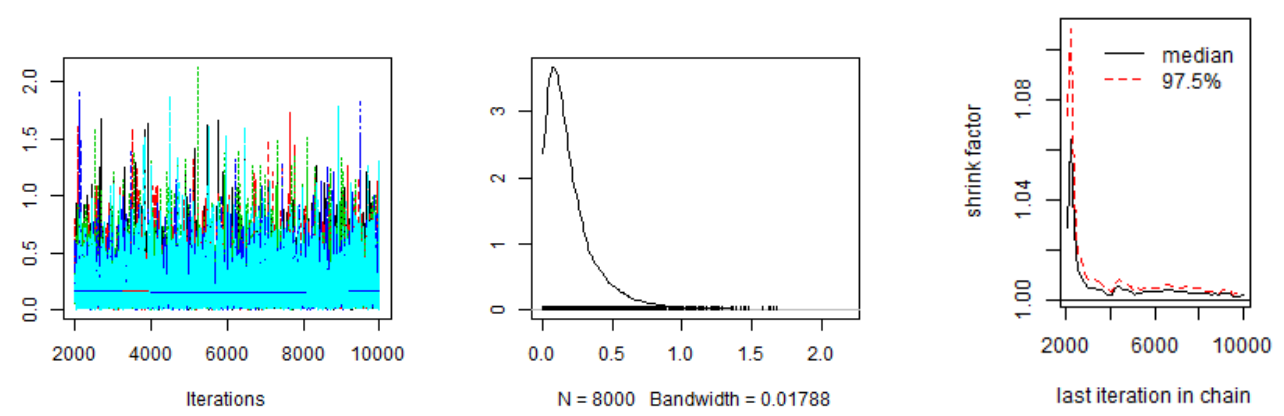

(a) Trace plot, density plot and Gelman-Rubin diagnostic plot.

Figure 24: Javelinfish (JAV): Convergence checks of posterior sample of $q$. 
For multiple chains the Gelman and Rubin diagnostic checks whether the within-chain variance of a parameter equals the total variance across all chains via a weighted sum of the within- and between-chain variance, the scale reduction parameter, $\hat{R}$ [21]. Values of $\hat{R}$ close to 1 suggest good convergence (all but one chain tested), while those above 1.2 indicate there may be problems with convergence which may be the case with parameter $K$ for Oblique banded rattail.

The Heidelberger and Welch diagnostic calculates a test statistic to accept or reject the null hypothesis that the Markov chain is from a stationary distribution. The test statistic is first calculated on the entire chain. If the null hypothesis is rejected, the first $10 \%$ of the chain is discarded and the test statistic recalculated on the remaining chain. This process is repeated until the null hypothesis is accepted or $50 \%$ of the chain has been discarded, in which case the chain fails the test and needs to be run for longer. One out of the five chains failed for parameter $K$ for Javelinfish and Oblique banded rattail, otherwise all the other chains passed this test.

In general our convergence tests were satisfied for the multiple chain setup. However, due to some questionable results for parameter $K$ we based parameter inference on samples from a single chain that was run for longer than the multiple chains. We ran the single chain for 15 million iterations, discarding the first 5 million and thinning the remaining samples by 500, resulting in 20,000 samples for inference purposes. We also checked trace plots and conducted the Heidleberger and Welch diagnostic as a convergence check for the single chain.

To test sensitivity to the choice of prior parameters, we ran a series of nine simulations (for each species) where we specified $E(r)$ at three different levels $(0.1,0.4,0.8)$ for the lognormal prior for $r$, and the upper limit for the uniform prior on $K$ at three different levels: $(\max ($ Catch $) \times 20$, $\max ($ Catch $) \times 100$, and $\max ($ Catch $) \times 1000$.

\subsection{Results}

The posterior mean, median and 95\% HPD interval of the model parameters $\left(r, K, q, \sigma_{u}^{2}, \sigma_{v}^{2}\right)$ from the nine simulations for Javelinfish data are listed in Table 5.3. Due to the skewed nature of the posterior distributions, we take the median (rather than the mean) as the parameter estimate and hence the 95\% HPD interval (rather than the symmetric credible interval). We plotted the posterior distribution for $r$ (Figure 25) and $K$ (Figure 26) 
for each simulation along with the corresponding prior distribution.

Table 5.3: Javelinfish (JAV): Posterior estimates

\begin{tabular}{|c|c|c|c|c|c|c|c|c|c|}
\hline & \multicolumn{3}{|c|}{$r$ prior: $\mathrm{E}(r)=0.1$} & \multicolumn{3}{|c|}{$r$ prior: $\mathrm{E}(r)=0.4$} & \multicolumn{3}{|c|}{$r$ prior: $\mathrm{E}(r)=0.8$} \\
\hline & & & $95 \%$ & & & $95 \%$ & & & $95 \%$ \\
\hline & mean & median & HPD interval & mean & median & HPD interval & mean & median & HPD interval \\
\hline \multicolumn{10}{|c|}{$K$ prior: upper limit $\max (\mathrm{C}) \times 20$} \\
\hline$r$ & 0.15 & 0.09 & $(0.01,0.48)$ & 0.37 & 0.33 & $(0.09$ & 0.73 & 0.7 & $(0.35,1.14)$ \\
\hline$K$ & 168 & 122 & $(15,449)$ & 94 & 71 & & 52 & 42 & \\
\hline$q$ & 0.1763 & 0.1306 & $(0.0087,0$ & 0.2696 & 0.2111 & $(0.0157,0.6766)$ & 0.4099 & 0.3259 & $(0.0302,1.029)$ \\
\hline$\sigma_{u}^{2}$ & 0.1612 & 0.1513 & $(0.0003,0$. & 0.1636 & 0.1551 & $(0.0002,0.3$ & 0.1752 & 0.1711 & $(0.0002,0.3347)$ \\
\hline$\sigma_{v}^{2}$ & 0.0544 & 0.0187 & $(0.0001,0.2476)$ & 0.0542 & 0.0135 & $(0.0002,0.2$ & 0.0518 & 0.0039 & $(0.0001,0.2904)$ \\
\hline MSY & 4.2 & 2.9 & $(0.1,11.9)$ & 7.5 & 6 & $(1.2,1$ & 8.8 & 7.5 & $(2.6,17.9)$ \\
\hline \multicolumn{10}{|c|}{$K$ prior: upper limit $\max (\mathrm{C}) \times 100$} \\
\hline$r$ & 0.1 & 0.06 & 1 & $2+2>$ & 0.27 & 0 & 0.7 & 0.68 & $(0.3$ \\
\hline$K$ & 561 & & & 240 & & & 83 & 50 & \\
\hline$q$ & 0.0955 & 9 & .001, & 1808 & 0.1304 & $(0.002$ & 447 & 0.2722 & $(0.0066,0.8979)$ \\
\hline$\sigma_{u}^{2}$ & 0.1636 & 0.1543 & $(0.0003$ & 0.1626 & 0.1549 & $(0.0003,0.3126)$ & 0.1744 & 0.1706 & $(0.0002,0.3336)$ \\
\hline$\sigma_{v}^{2}$ & 0.0466 & 0.0139 & $(0.0001,0.2156)$ & 0.051 & 0.0118 & $(0.0001,0.2492)$ & 0.051 & 0.0036 & $(0.0001,0.2887)$ \\
\hline MSY & 9.6 & 4.2 & $(0.1,32.9)$ & 15.3 & 8.1 & $(0.8,46.7)$ & 13.1 & 8.5 & $(2.2,29.2)$ \\
\hline \multicolumn{10}{|c|}{$K$ prior: upper limit $\max (\mathrm{C}) \times 1000$} \\
\hline$r$ & 0.08 & 005 & - & 0 & & & 0. & 0.67 & $(0$. \\
\hline$K$ & 1948 & 527 & & 935 & 167 & (1 & 110 & 53 & $(12$ \\
\hline$q$ & 0.0553 & 0.0287 & $(0.0004$ & 0.1379 & 0.0865 & $(0.0004$ & 0.3308 & 0.258 & $(0.0076,0.8828)$ \\
\hline$\sigma_{u}^{2}$ & 0.1644 & 0.1546 & $(0.0006,0.3074)$ & 0.1596 & 0.1516 & $(0.0003,0.3083)$ & 0.1698 & 0.1666 & $(0.0002,0.3297)$ \\
\hline$\sigma_{v}^{u}$ & 0.0428 & 0.0128 & $(0.0001,0.1944)$ & 0.054 & 0.0138 & $(0.0001,0.2598)$ & 0.0597 & 0.0041 & $(0.0001,0.3163$ \\
\hline MSY & 30.5 & 7.2 & $(0.1,120.3)$ & 54.9 & 10.9 & $(0.8,236)$ & 16.8 & 8.9 & $(2.3,46.5)$ \\
\hline
\end{tabular}

The posterior distribution of $r$ is similar to the prior distribution and is dependent on the value specified for $E(r)$ in the prior, as shown in the plots from left to right in Figure 25. This indicates that there is little information in the data to inform parameter estimates for $r$, and that the prior is more dominant than the likelihood in estimating $r$. This trend for $r$ is repeated for increasing upper-limits specified in the $K$ prior. That is, there is little difference in this trend when the maximum level of $K$ is increased in the prior for $K$, shown in the plots from top to bottom. Although, for a fixed $r$ prior, increasing the upper-limit in the $K$ prior does reduce the posterior median (and mean) for $r$ slightly. Altogether, this suggests that parameter estimates for $r$ are not sensitive to the $K$ prior. However, they are sensitive to the prior choice of $E(r)$ as shown by the increase in the posterior median of $r$ with prior $E(r)$.

The posterior distribution of $K$ reflects the prior specification of $K$ and $r$. For a fixed $r$ prior specification, as the upper-limit in the $K$ prior is increased, the posterior median for $K$ increases, as does the upper-bound for the posterior 95\% HPD interval on $K$. However, when the value of $E(r)$ set in the $r$ prior increases, the posterior median for $K$ decreases for 
a given upper-limit in the $K$ prior. And, for higher values of $E(r)$ set in the $r$ prior, the effect that increasing the upper-limit set in the $K$ prior has on increasing the posterior median for $K$ diminishes (considerably so, at $E(r)=0.8$ compared to lower values set in the $r$ prior).

Figure 27 contains bivariate scatterplots for the parameters $r, K$ and $q$, for each of the 9 simulations. Parameters $K$ and $q$ are negatively correlated (lower $q$ values with higher $K$ values) as we would expect for the scale parameters (right plot in each section). Parameters $r$ and $q$ tend not to be highly correlated (middle plot in each section), but parameters $r$ and $K$ are negatively correlated.

To gauge the impact of the different parameters estimated in each simulation we plot the observed index $I$ and the predicted index $\hat{I}$ with $95 \%$ HPD interval (Figure 28) and the predicted biomass $\hat{B}$ with 95\% HPD interval (Figure 29). The fit of the predicted index $\hat{I}$ to the observed index $I$ does not change appreciably for the different prior specifications. However, the results from for the predicted Biomass, $\hat{B}$, suggests that the predictions are driven mainly by information from the prior distributions. The estimated biomass is scaled according to the confounding of parameters $r, K$ and $q$. For a fixed prior on $K$, as the value of $E(r)$ is increased in the prior for $r$, the posterior estimate for biomass $B_{t}$ decreases. And for a fixed prior on $r$, as the upper-limit in the $K$ prior is increased, the posterior estimate for biomass $B_{t}$ also increases, as does the parameter uncertainty (i.e. upperbound of the 95\%HPD interval increases). So, for different combinations of priors on $r$ and $K$ (e.g. $E(r)=0.1$ in the $r$ prior, and upper-limit in the $K$ prior set at $\operatorname{mac}(C) \times 1000$ ) we could report that the absolute population biomass for Javelinfish, as at the end of the 2012 fishing year, was around 400,000 tonnes, with a lower limit close to 0 and an upper limit of over 1 million tonnes. For an equally well fitted model (e.g. $E(r)=0.4$ in the $r$ prior, and upper-limit in the $K$ prior set at $\operatorname{mac}(C) \times 20$ ), we could also report that the population biomass was about 40,000 tonnes, with a lower limit close to 0 and an upper limit of 100,000 tonnes. Similarly varying results are evident for models applied to the other three rattail species data (Appendix K).

These levels of uncertainty mean that considerable thought needs to be given to using them for informing management decisions of these rattail species. Improving data quality, refining informative priors, or changing the model specification (or all three) may help to improve parameter estimates and reduce uncertainty. 

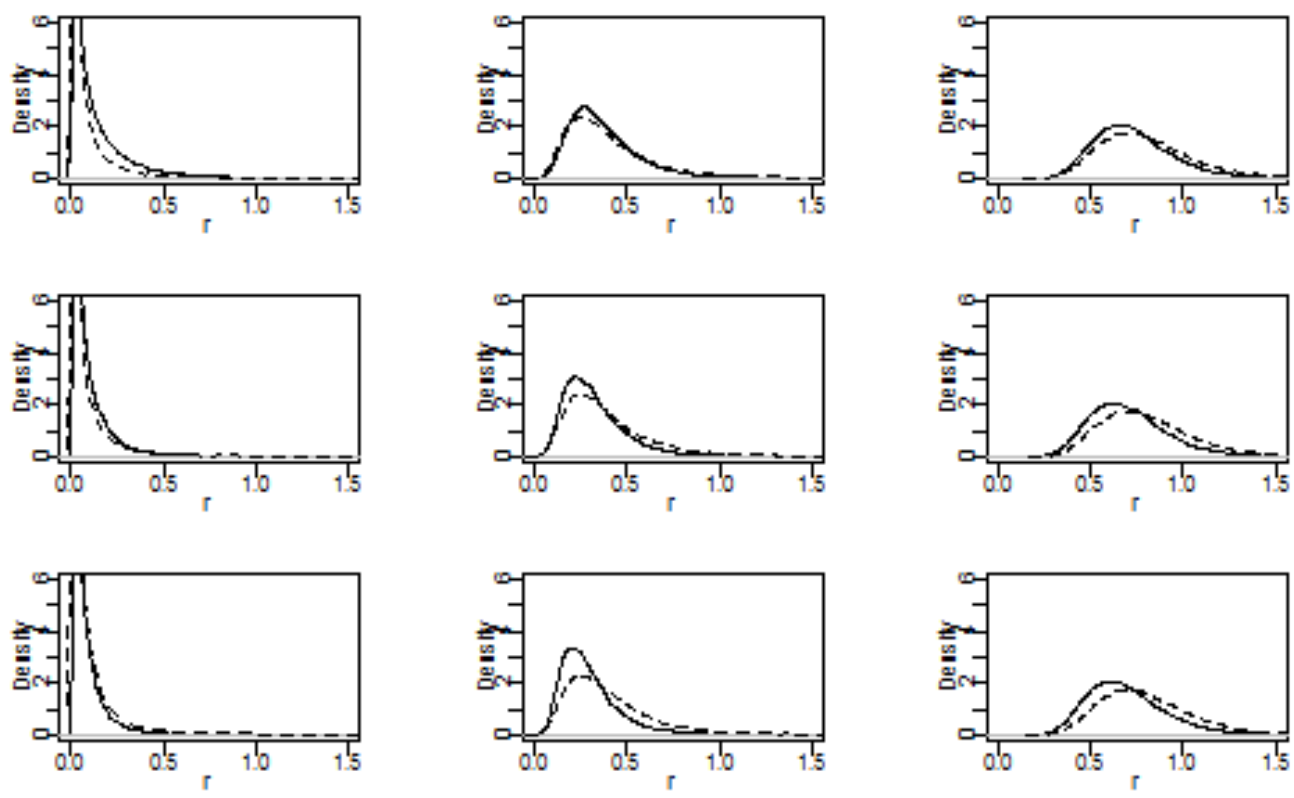

Figure 25: Javelinfish (JAV): prior and posterior distributions for $r$. Solid line is posterior distribution, dashed line is prior distribution. Left to right: increasing value of $\mathrm{E}(r)=$ $(0.1,0.4,0.8)$ specified in $r$ prior. Top to bottom: increasing upper-limit set in $K$ prior $(\max (C) \times 20, \max (C) \times 100, \max (C) \times 1000)$.
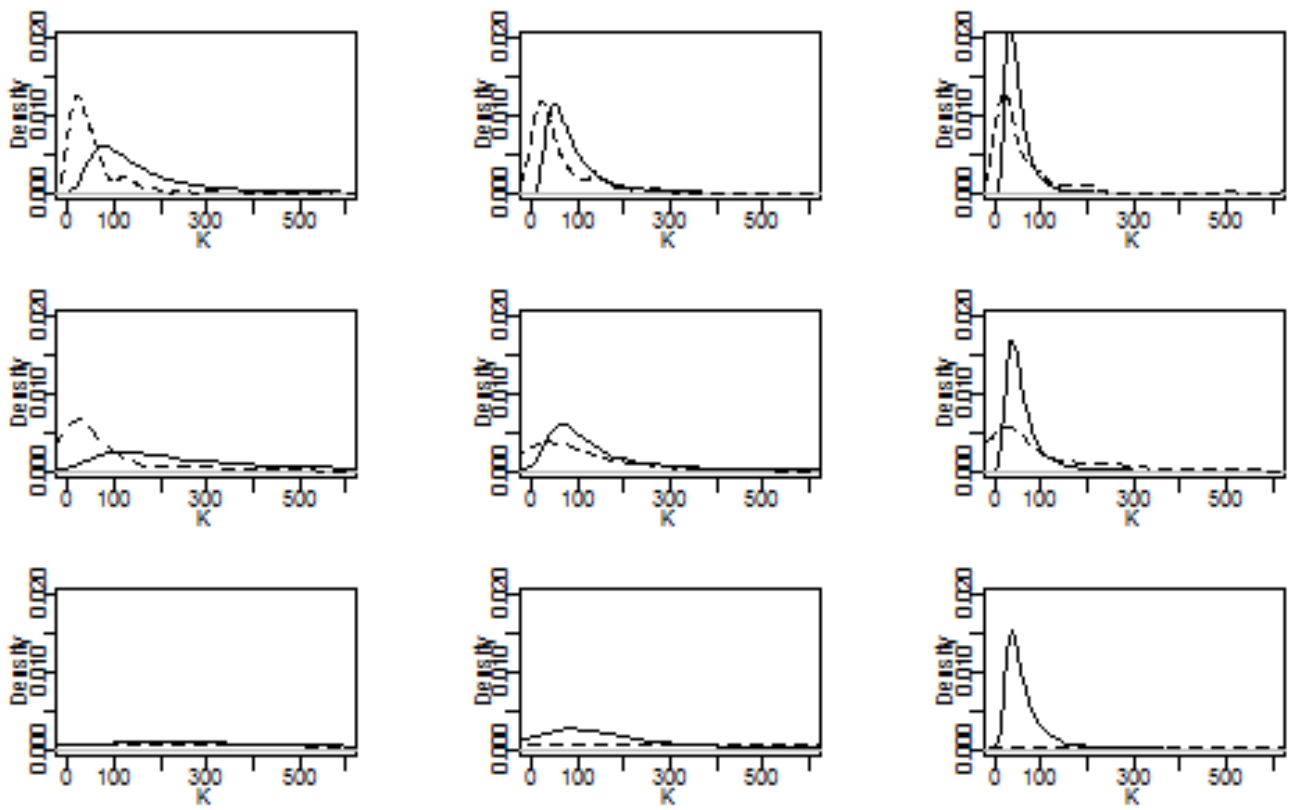

Figure 26: Javelinfish (JAV): prior and posterior distributions for $K$. Solid line is posterior distribution, dashed line is prior distribution. Left to right: increasing value of $\mathrm{E}(r)=$ $(0.1,0.4,0.8)$ specified in $r$ prior. Top to bottom: increasing upper-limit set in $K$ prior $(\max (C) \times 20, \max (C) \times 100, \max (C) \times 1000)$. 


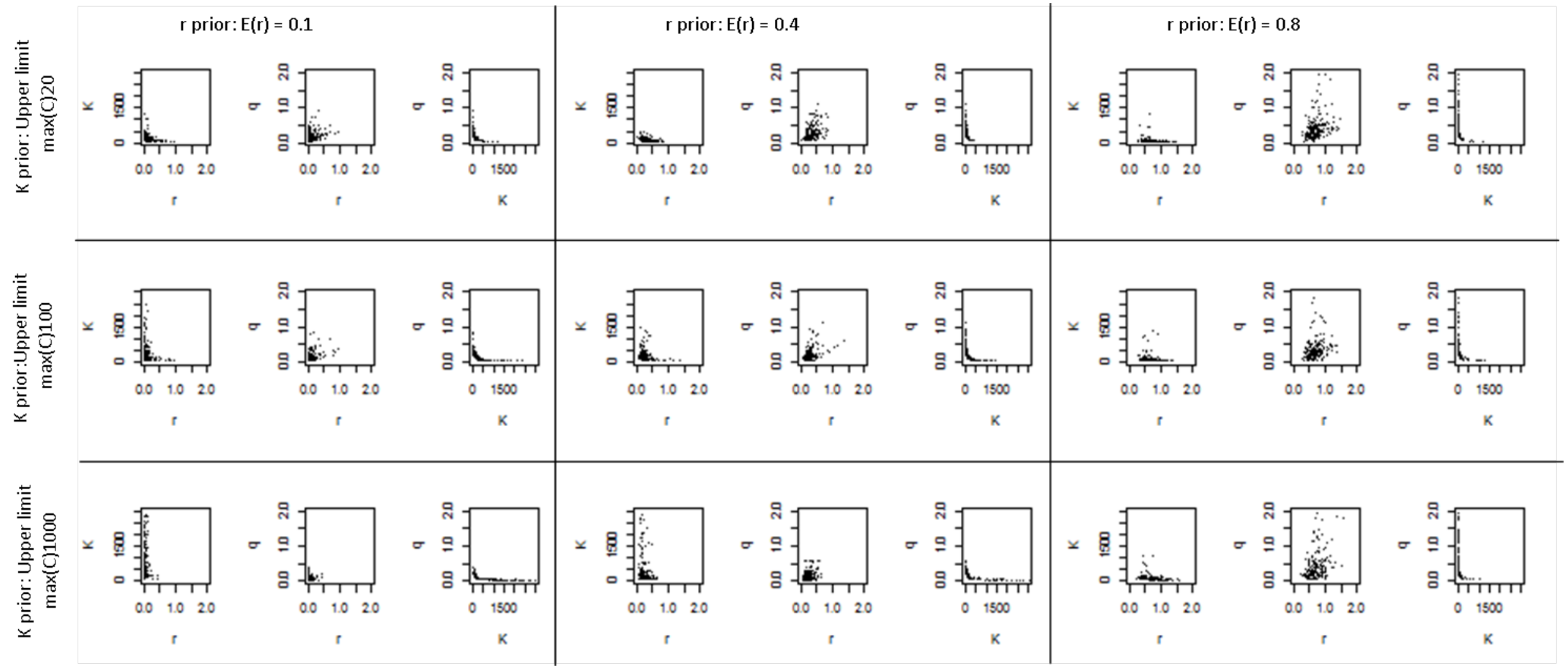

Figure 27: JAV: parameter correlations $r, \mathrm{~K}, \mathrm{q}$ 

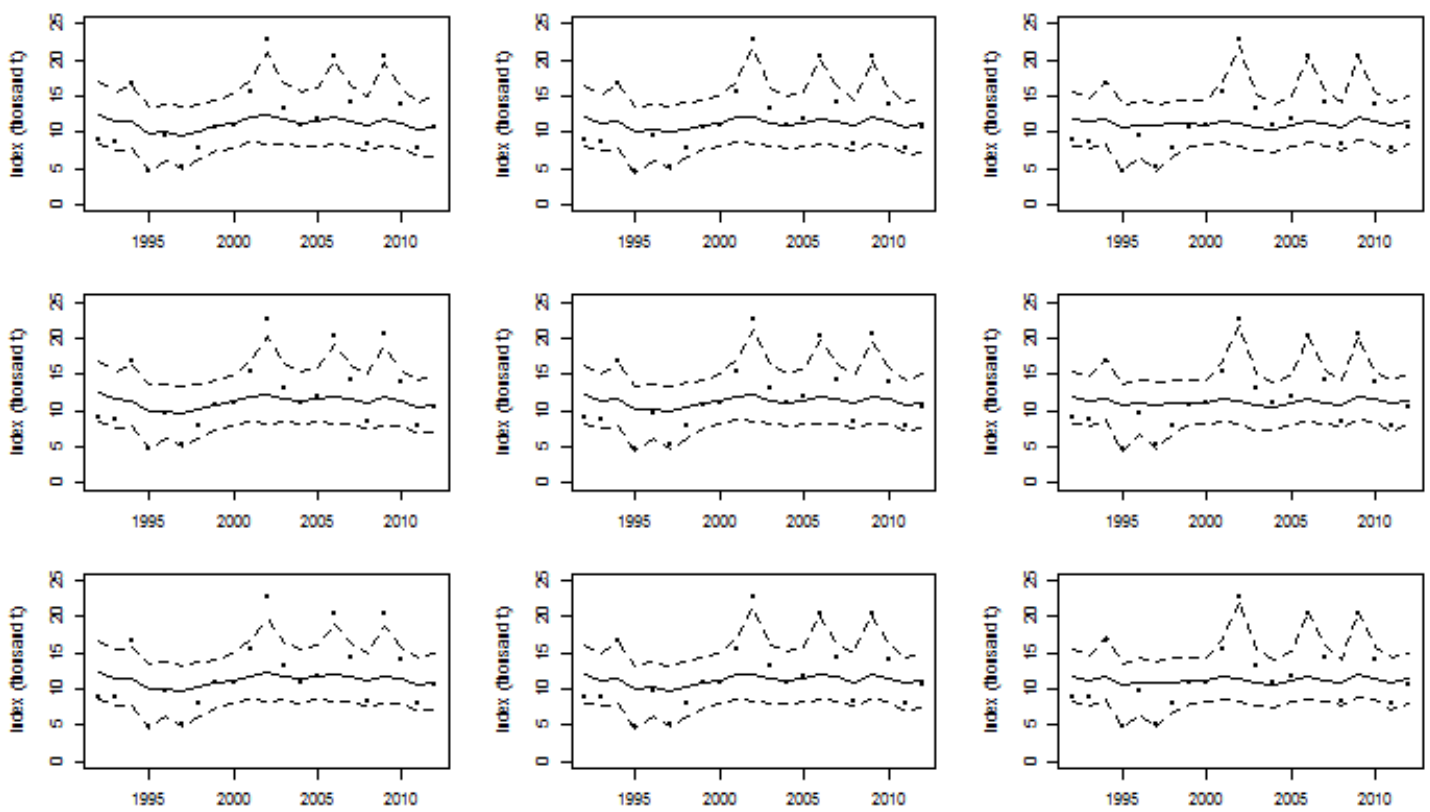

Figure 28: Javelinfish (JAV): Plot of observed index $\left(I_{t}=\bullet\right.$ ) and posterior median for the model predicted index (solid line) with its 95\%HPD interval (dashed line).Left to right: increasing value of $\mathrm{E}(r)=(0.1,0.4,0.8)$ specified in $r$ prior. Top to bottom: increasing upperlimit set in $K$ prior $(\max (C) \times 20, \max (C) \times 100, \max (C) \times 1000)$.
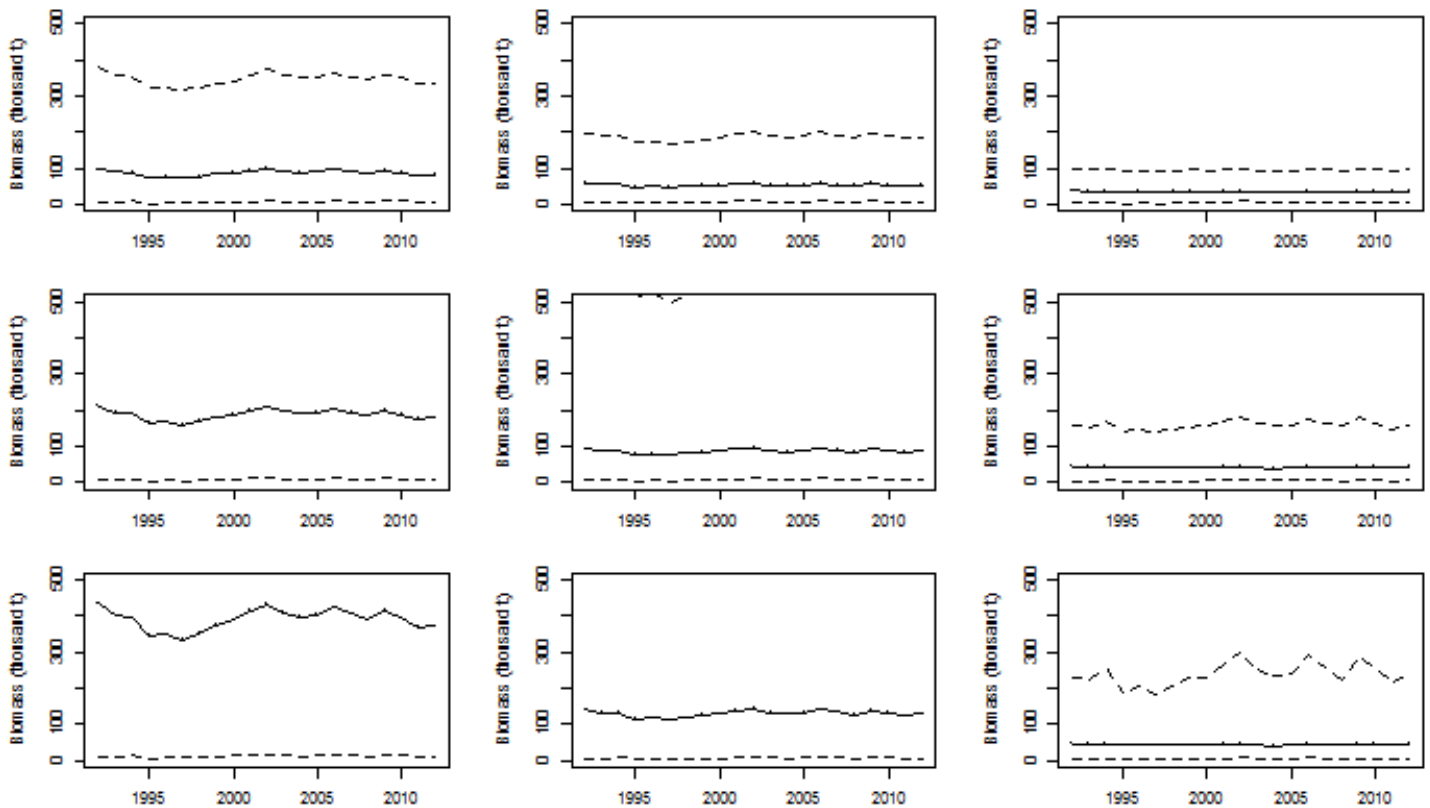

Figure 29: Javelinfish (JAV): Plot of the posterior median for the model predicted biomass (solid line) with its 95\%HPD interval (dashed line). Left to right: increasing value of $\mathrm{E}(r)=$ $(0.1,0.4,0.8)$ specified in $r$ prior. Top to bottom: increasing upper-limit set in $K$ prior $(\max (C) \times 20, \max (C) \times 100, \max (C) \times 1000)$. 


\section{Chapter 6}

\section{Discussion}

In this project we conducted a stock assessment for four rattail species (bycatch for the hoki fishery on the Chatham Rise) using a surplus production model. We reconstructed the catch history for the rattails using effort data from the commercial fishery and catch/effort data from scientific surveys. We fitted an observation model using maximum likelihood estimation. In addition we estimated model parameters for an observation- and processerror model using Bayesian methods. In the surplus production model, we treated catch, $C$ as observed without error, thus ignoring uncertainty associated with the reconstruction estimate of these catches. As a result, the confidence intervals for the model parameters could be wider than those we presented.

The maximum likelihood method (at best) fitted straight lines through the data and failed to account for any variation, the parameters being estimated with high uncertainty. Parameter estimates were unstable for different MLE procedure types, resulting in similar model fits, but with quite different parameter estimates. This result is not surprising given the number of paramters to be esitmated from very few observations, and the lack of contrast evident in the time series of observartions. The Bayesian approach was considered more plausible for the small sample size, and for the opportunity to introduce prior information.

Estimation was improved slightly with the Bayesian method using an informed prior for parameter $r$, the intrinsic population growth rate. While the use of an uninformed prior would have been appropriate for $K$, the carrying capacity, we chose to set a prior for $K$ that limited implausibly large posterior values being estimated for this scale parameter.

We conducted sensitivity analysis to see how much the posterior infer- 
ences change when different prior densities are assumed. Keeping all other prior distributions unchanged, we changed the mean values within the lognormal prior for $r$. We found that the posterior inferences for $r$ reflected the specified prior. The parameters were set in the prior on $K$ so as to have little influence on the posterior medians. However, we found that increasing the variance parameter (based on an interval with increasing upper-limits) resulted in higher posterior medians with greater associated uncertainty. Larger mean values specified in the $r$ prior mitigated this effect.

The sensitivity of the posterior inference to the prior distribution of $r$ and $K$, leading to the inability of this method to estimate $K$ with any certainty, along with the confounding of the parameters $r, K$ and $q$, indicates the lack of information in the data. And, as this data is lacking about one of the most important yield determining factors it is a constraint that must be accounted for in making decisions about managing these rattail populations [79].

Future work could include:

- Investigating the robustness of the catch reconstruction method and determining the error structure associated with this estimation process.

- Considering a surplus production model that allows for error in the catch predictor, rather than treating catch as observed without error.

- Investigating methods of constructing informative priors for the intrinsic growth rate, $r$, (e.g. McAllister et. al [61] use demographic analysis to construct a prior for $r$ ).

- Investigating hierarchical models, as proposed for data-poor situations. With a hierarchical Bayesian approach all the species can share some identical parameters. It is suggested for species-specific data, where species with uninformative data "borrow strength from species with good data" [51]. We started this work on the 4 rattail species, setting a shared prior for $r$. Preliminary results suggest this method does not offer significant improvements for parameter estimation. There is lack of information in the data overall and the benefits of "borrowing" information from other species is not evident.

- Incorporating environmental variables into the model. This method is particularly recommended when fishing effort variations explain only a small part of the total variability in annual catches [37]. The 
abundance of the stock or the catchability may be affected by some environmental variable such as predator-prey interactions or climate variation. For example, Rose [83] applies a scalar, in the form of a normalised index of climate (based on tree ring growth) to the parameter $r$ in the population dynamics (process) part of the surplus production model. 


\section{References}

[1] Abraham, E. R., Thompson, F. N., And Oliver, M. D. Summary of the capture of seabirds, marine mammals, and turtles in New Zealand commercial fisheries, 1998-99 to 2007-08. New Zealand Aquatic Environment and Biodiversity Report No. 45. (2010), 148. http: //fs.fish.govt.nz/Doc/22138/AEBR_45.pdf.ashx.

[2] ANDERSON, O. F. Fish discards and non-target fish catch in the fisheries for southern blue whiting and oreos. New Zealand Fisheries Assessment Report 2004/9. (2004), 40p.

[3] Anderson, O. F. Fish discards and non-target fish catch in the trawl fisheries for arrow squid, jack mackerel, and scampi in New Zealand waters. New Zealand Fisheries Assessment Report 2004/10. (2004), 61p.

[4] Anderson, O. F. Fish discards and non-target fish catch in the New Zealand jack mackerel trawl fishery 2001-02 to 2004-05. New Zealand Aquatic Environment and Biodiversity Report No. 8. (2007), 36p.

[5] Anderson, O. F. Fish discards and non-target fish catch in the New Zealand orange roughy trawl fishery 1999/2000 to 2004/05. New Zealand Aquatic Environment and Biodiversity Report No. 39. (2009), 40p. http://fs.fish.govt.nz/Doc/22052/AEBR_39.pdf.ashx.

[6] Anderson, O. F. Fish and invertebrate bycatch and discards in orange roughy and oreo fisheries from 1990-91 until 2008-09. New Zealand Aquatic Environment and Biodiversity Report No. 67. (2011), 60p. http://fs.fish.govt.nz/Doc/22866/AEBR_67.pdf.ashx.

[7] ANDERson, O. F. Fish and invertebrate bycatch and discards in New Zealand's scampi fisheries from 1990-91 until 2009-10. New Zealand Aquatic Environment and Biodiversity Report No. 100. (2012), 65p. http://fs.fish.govt.nz/Doc/23066/AEBR_100.pdf. ashx. 
[8] Anderson, O. F., Gilbert, D. J., And Clark, M. R. Fish discards and non-target catch in the trawl fisheries for orange roughy and hoki in New Zealand waters for the fishing years 1990-91 to 1998-99. New Zealand Fisheries Assessment Report 2001/16. (2001), 57p.

[9] Anderson, O. F., AND SMith, M. H. Fish discards and non-target fish catch in the New Zealand hoki trawl fishery, 1999-2000 to 200203. New Zealand Fisheries Assessment Report 2005/3. (2005), 37p.

[10] AzZAlini, A. Statistical inference based on the likelihood. Chapman and Hall, London, 1996.

[11] Ballara, S. L., AND ANDerson, O. F. Fish discards and non-target fish catch in the trawl fisheries for arrow squid and scampi in New Zealand waters. New Zealand Aquatic Environment and Biodiversity Report No. 38. (2009), 102. http://fs.fish.govt.nz/Doc/22289/ AEBR_38.pdf.ashx.

[12] Ballara, S. L., AND O'Driscoll, R. L. Catches, size, and age structure of the 2010-11 hoki fishery, and a summary of input data used for the 2012 stock assessment. New Zealand Fisheries Assessment Report 2012/23. (2012), 117. http: / f s. fish. govt.nz /Doc/ 23016/12_23_FAR.pdf.ashx.

[13] Ballara, S. L., O’Driscoll, R. L., And Anderson, O. F. Fish discards and non-target fish catch in the trawl fisheries for hoki, hake, and ling in New Zealand waters. New Zealand Aquatic Environment and Biodiversity Report No. 48. (2010), 100p. http://fs.fish. govt.nz/Doc/22289/AEBR_48.pdf.ashx.

[14] BentLey, N., AND STOKES, K. Contrast paradigms for fisheries managment decision making: how well do they serve data-poor fisheries. Marine and Coastal Fisheries: Dynamcis, Management and Ecosystem Science 1 (2009), 391-340. doi. 10.1577/C08-044.1.

[15] BolKeR, B. M. Ecological Models and Data in R. Princeton University Press, Princeton, 2008.

[16] BOLKer, B. M. Package 'emdbook'. Ecological models and data (book support). Version 1.3.4, 2013. http: //cran.r-project.org/web/ packages/emdbook/emdbook.pdf.

[17] BONFIL, R. Fishery stock assessment models and their application to sharks. In FAO Fisheries Technical Paper 474: Management techniques for elasmobranch fisheries, J. A. Musick and R. Bonfil, Eds. FAO, 
Rome, 2005, pp. 154-181. ftp://ftp.fao.org/docrep/fao/ $008 / a 0212 \mathrm{e} / \mathrm{a} 0212 \mathrm{E} 00 . \mathrm{pdf}$.

[18] BrodziaC, J., AND IshimurA, G. Development of Bayesian production models for assessing the North Pacific swordfish population. Fish Science 77 (2011), 23-34. doi. 10.1007/s12562-010-0300-0.

[19] Bull, B., Francis, R. I. C. C., DunN, A., MCKenzie, A., Gilbert, D. J., SMITH, M. H., BIAN, R., AND FU, D. CASAL (C++ algorithmic stock assessment laboratory): CASAL User Manual v2.302012/03/21. NIWA Technical Report 135 (2012), 280p.

[20] Caillet, G., Devine, J., Drazen, J., Duran, P., Bezaury, J., AND Orlov, A. Sustainable deep-sea fisheries for grenadiers. In Can Ecosystem-Based Deep-Sea Fishing Be Sustained? Report of a workshop held 31 August-3 September 2010. Darling Marine Center Special Publication 11-1., L. Watling, R. Haedrich, J. Devine, J. Drazen, and M. Dunn, Eds. University of Maine, Darling Marine Center., Walpole, ME, 2011, p. 84. http://digitalcommons.library.umaine.edu/cgi/ viewcontent.cgi?article $=1144 \&$ context=sms_facpub.

[21] Carlin, B. P., AND Thomas, A. L. Bayesian Methods for Data Analysis, 3rd ed. Chapman and Hall, Boca Raton, 2009.

[22] De Leo, F. C., Smith, C. R., Rowden, A. A., Bowden, D. A., AND CLARK, M. R. Submarine canyons: hotspots of benthic biomass and productivity in the deep sea. Proceedings of the Royal Society B: Biological Sciences 277, 1695 (2010), 2783-2792.

[23] De VAlpine, P., AND Hilborn, R. State-space likelihoods for nonlinear fisheries time-series. Canadian Journal of Fisheries and Aquatic Science 62 (2005), 1937-1952.

[24] Devine, J. A., Watling, L., Cailliet, G., Drazen, J., DuRAN MunOz, P., ORlov, A. M., AND BEZAury, J. Evaluation of potential sustainability of deep-sea fisheries for grenadiers (Macrouridae). Journal of Ichthyology 52, 10 (2012), 709-721. doi: 10.1134/S0032945212100062.

[25] Dunn, A., Harley, S. J., Doonan, I. J., And Bull, B. Calculation and interpretation of catch-per-unit-effort (CPUE) indices. New Zealand Fisheries Assessment Report 2000/1. (2000), 44p. http: //docs.niwa.co.nz/library/public/FAR2001-01.pdf. 
[26] DunN, M. R. A review of experimental methods for determining catchability for trawl surveys. New Zealand Fisheries Assessment Report 20006/51. (2006), 31p.

[27] EFron, B. The jackknife, the bootstrap, and other resampling plans., vol. 38 of CBMS Regional Conference Series in Applied Mathematics. Society for Industrial and Applied Mathematics, 1982.

[28] EFron, B., AND TibShiRAni, R. J. An Introduction to the Bootstrap. Chapman and Hall, New York, 1993.

[29] Fox, W. W. An exponential surplus-yield model for optimizing exploited fish populations. Transactions of the American Fish Society 99, 1 (1970), 80-88.

[30] Francis, M. P., Hurst, R. J., McArdle, B. H., Bagley, N. W., AND ANDERSON, O. F. New Zealand demersal fish assemblages. Environmental Biology of Fishes. 65, 2 (2002), 215-234.

[31] FRANCIS, R. I. C. C. Stratified random trawl surveys of deep-water demersal fish stocks around New Zealand. Fisheries Research Division Occasional Publication No. 32 (1981), 28p. http://docs. niwa.co. nz/library/public/FRDop32.pdf.

[32] FRANCIS, R. I. C. C. An adaptive strategy for randomised trawl surveys. New Zealand Journal of Marine and Frishwater Research. 18 (1984), 59-71. doi: 10.1080/00288330.1984.9516030.

[33] FRANCIS, R. I. C. C. A standard approach to biomass estimation from bottom trawl surveys. New Zealand Fisheries Assessment Research Document 89/03 (1989), 4p. http: / / docs.niwa.co.nz/library/ public/89_03_FARD.pdf.

[34] Francis, R. I. C. C. Optimum allocation of stations to strata in trawl surveys. New Zealand Fisheries Assessment Report 2006/23. (2006), 50p. http://fs.fish.govt.nz/Doc/10635/2006\%20FARs/ 06_23_FAR.pdf.ashx.

[35] Francis, R. I. C. C. Data weighting in statistical stock assessment models. Canadian Journal of Fisheries and Aquatic Sciences 68, 6 (2011), 1124-1138.

[36] Francis, R. I. C. C., Hurst, R., And Renwick, J. A. An evaluation of catchability assumptions in New Zealand stock assessments. New Zealand Fisheries Assessment Report 2001/1. (2001), 38p. http: //docs.niwa.co.nz/library/public/FAR2001-01.pdf. 
[37] FREON, P. Introduction of climatic variables into global production models. In International Symposium on Long Term Changes in Marine Fish Populations. Vigo, Spain, 1986, T. Wyatt and M. G. Larraneta, Eds. Consejo Superior de Investigaciones Cientificas, 1989, pp. 481-528.

[38] Gelfand, A., AND SMITH, A. Sampling-based approaches to calculating marginal densities. Journal of the American Statistical Association 85, 410 (1990), 398-409. http:/ / www.jstor.org/stable/2289776.

[39] Gelman, A., Carlin, J. B., Stern, H. S., And Rubin, D. B. Bayesian Data Analysis. Chapman and Hall, London, 1995.

[40] GELMAN, A., AND RUBIN, D. B. Inference from iterative simulation using multiple sequences. Statistical Science 7, 4 (1992), $457-472$. http: //www.jstor.org/stable/2246093.

[41] GiBsON, M. Assessing the local population of winter flounder with a two-era biomass dynamic model: a narrower view of southern New England. Rhode Island Division of Fish and Wildlife, Marine Fisheries Office, Jamestown (2013), 82p.

[42] HadDon, M. Modelling and Quantitative Methods in Fisheries, 2nd ed. CRC Press, Boca Raton, 2011.

[43] Hastings, W. Monte carlo sampling methods using markov chains and their applications. Biometrika 57,1 (1970), 97-109. http: / / www . jstor.org/stable/2334940.

[44] Heidelberger, P., AND WelCH, P. D. Simulation run length conrol in the presence of an initial transient. Operations Research 31, 6 (1983), 1109 -1144. http: //www. jstor.org/stable/170841.

[45] Hilborn, R. Comparison of fisheries control systems that utilize catch and effort data. Journal of the Fisheries Research Board of Canada 36 (1979), 1477-1489.

[46] Hilborn, R., AND MAngel, M. The Ecological Detective: Confronting Models with Data. Monographs in Population Biology. Princeton University Press, Princeton, 1997.

[47] Hilborn, R., AND Walters, C. J. Quantitative Fisheries Stock Assessment: Choice, Dynamics and Uncertainty. Chapman and Hall, NY, 1992. 
[48] Hurst, R. J., Bagley, N., Chatterton, T., Hanchet, S., SCHOFIELD, K., AND VignAuX, M. Standardisation of hoki/middle depth time series trawl surveys. MAF Fisheries Greta Point Internal Report No. 194. (1992), 89p. http://docs.niwa.co.nz/library/ public/IRMAFFGP 194 .pdf.

[49] IWAmOtO, T. Family Macrouridae. In FAO species catalogue. Vol. 10. Gadiform fishes of the world (Order Gadiformes). An annotated and illustrated catalogue of cods, hakes, grenadiers and other gadiform fishes known to date., D. Cohen, T. Inada, T. Iwamoto, and N. Scialabba, Eds. FAO, Rome, 1990, pp. 90-317.

[50] IWAmOTO, T. A brief taxonomic history of grenadiers. In Grenadiers of the world oceans: biology, stock assessment and fisheries., A. M. Orlov and T. Iwamoto, Eds. American Fisheries Socieity Symposium 63, Bethesda, 2008, pp. 3-13.

[51] Jiao, Y., Cortes, E., Andrews, K., And Guo, F. Poor-data and data-poor species stock assessment using bayesian hierarchical approach. Ecological Applications 21, 17 (2011), 2691-2708.

[52] Livingston, M. E., Clark, M. R., And Baird, S. J. Trends and incidental catch of major fisheries on the Chatham Rise for fishing years 1989-90 to 1998-99. New Zealand Fisheries Assessment Report 2003/52. (2003), 74p. http://docs.niwa.co.nz/library/ public/FAR2003-52.pdf.

[53] Loreau, M. From Populations to Ecosystems. Princeton University Press, Princeton, 2010.

[54] LudwiG, D., AND WALTers, C. J. A robus method for parameter estimation from catch and effort data. Canadian Journal of Fisheries and Aquatic Sciences 46, 1 (1989), 137-144.

[55] Lunn, D. J., Thomas, A., Best, N., And Spiegelhalter, D. WinBUGS, a Bayesian modelling framework: concepts, structure, and extensibility. Statistics and Computing 10, 4 (2000), 325-337. doi: 10.1023/A:1008929526011.

[56] MACE, P. M. A new role for MSY in single-species and ecosystem approaches to fisheries stock assessment and management. Fish and Fisheries 2 (2001), 2-32. http:/ / onlinelibrary.wiley.com.helicon.vuw.ac.nz/doi/10.1046/j.14672979.2001.00033.x/pdf. 
[57] MACE, P. M. In defence of fisheries scientists, single-species models and othe scapegoats: confronting the real problems. Marine Ecology Progress Series 274 (2004), 285-291.

[58] Magnusson, A., AND Hilborn, R. What makes fisheries data informative? Fish and Fisheries 8 (2007), 337-358.

[59] McAllister, M., And DupliseA, D. E. Production model fitting and projection for Acadian redfish (Sebastes fasciatus) in Units 1 and 2. DFO Canadian Science Advisory Scretariate Research Document 2012/103 (2012), 37p.

[60] MCAllister, M. K., AND KiRKWOOD, G. Bayesian stock assessment: a review and example application using the logistic model. ICES Journal of Marine Science 55 (1998), 1031-1060.

[61] McAllister, M. K., Pikitch, E. K., AND BABCOCK, E. A. Using demographic methods to construct bayesian priors for the intrinsic rate of increase in the schaefer model and implications fro stock reasonably. Canadian Journal of Fisheries and Aquatic Sciences 58 (2001), 1871-1890.

[62] McKenZIE, A. Assessment of hoki (Macruronus novaezelandiae) in 2012. New Zealand Fisheries Assessment Report 2012/27. (2013), 65p. http://fs.fish.govt.nz/Doc/23149/FAR_2013_27_ 2626_DEE2010-02HOKA, 200bj.1, 20CS2.pdf.ashx.

[63] Metropolis, N., Rosenbluth, A., Rosenbluth, M., Teller, A., AND TELLER, E. Equations of state calculations by fast computing machines. Journal of Chemical Physics 21, 6 (1953), 1087-1092. doi: 10.1063/1.1699114.

[64] Meyer, R., AND Millar, R. B. Bugs in Bayesian stock assessments. Canadian journal of Fisheries and Aquatic Science 56 (1999), 1078-1087. http: / / search.proquest. com. helicon.vuw. ac . $\mathrm{nz} /$ docview/219334690/fulltextPDF?accountid=14782.

[65] Millar, R. B., AND MEyer, R. WinBUGS code for fitting a state-space production model. [Computer software]. (1999). https://www.stat.auckland.ac.nz/ millar/Bayesian/ Surtuna.bugs.

[66] Millar, R. B., AND Meyer, R. Non-linear state space modelling of fisheries biomass dynamics by using Metropolis-Hastings withinGibbs sampling. Journal of the Royal Statistical Society: Series C (Applied Statistics) 49 (2000), 337-342. doi. 10.1111/1467-9876.00195. 
[67] Ministry fOr Primary Industries, N. Fisheries Assessment Plenary, May 2013: stock assessments and yield estimates. Compiled by the Fisheries Science Group, Ministry for Primary Industries, Wellington, New Zealand, 2013. 1357 p.

[68] Ministry fOR Primary Industries, N. Z. The Status of New Zealand's Fisheries 2012. 2012. http://fs.fish.govt. $\mathrm{nz} / \mathrm{Doc} / 23087$ / The $\% 20$ Status\%200f20New20 Zealand\% 20 fisheries $\div 202012$.pdf. ashx.

[69] Ministry OF FisHeries, N. Z. Bottom Fishery Impact Assessment: Bottom fishing activities fyb New Zealand vessels fishing in the high seas in the SPRFMO Area during 2008 and 2009. 2008. http://www.fish.govt.nz/NR/ rdonlyres/344F062B-5331-481B-ADD7-FBF244566A96/0/ NewZeal andBottomFishery ImpactAs ses smentv11 cDec20082 small. pdf.

[70] Ministry OF Fisheries, N. Z. National Fisheries Plan for Deepwater and Middle-depth Fisheries. Part 1B: Hoki. 2010. http://www.fish.govt.nz/NR/rdonlyres/ 1BF0E4A9-A3D6-45CC-9A7D-49978E09161E/0/NFP_ Deepwater_and_Middledepth_Fisheries_Part_1B_HOKI. pdf.

[71] O’Driscoll, R. L., MacGibbon, D., Fu, D., LyON, W., AND STEVENS, D. W. A review of hoki and middle-depth trawl surveys of the Chatham Rise, January 1992-2010. New Zealand Fisheries Assessment Report 2011/47. (2011), 72p. http: / / fs.fish.govt.nz/ Doc/22948/11_47_EAR.pdf.ashx.

[72] PAul, L. New Zealand Fishes: Identification, Natural History and Fisheries, 2nd ed. Reed Books, Auckland, 2000.

[73] Pella, J. J., And Tomlinson, P. K. A generalized stock production model. Bulletin of the Inter-American Tropical Tuna Commission 13, 3 (1969), 421-498. http: / / www. iattc.org/PDFFiles2/ Bulletins/Bulletin-Vol.13-No.3.pdf.

[74] Plummer, M., Best, N., Cowles, K., And Vines, K. Coda: Convergence diagnosis and output analysis for MCMC. $R$ News 6, 1 (2006), 7-11. http://CRAN.R-project.org/doc/Rnews / Rnews_2006-1.pdf. 
[75] Polacheck, T., Hilborn, R., And Punt, A. E. Fitting surplus production models: Comparing methods and measuring uncertainty. Canadian Journal of Fisheries and Aquatic Sciences 50 (1993), 2597-2607.

[76] Prager, M. H. Comparison of logistic and generalised surplusproduction models applied to swordfish, Xiphias gladius, in the north Atlantic Ocean. Fisheries Research 58 (2002), 41-57.

[77] Prager, M. H., AND Williams, E. H. From the golden age to the new industrial age: fishery modeling in the early 21st century. Natural Resource Modeling 16 (2003), 487-489.

[78] PunT, A. E. Extending production models to include process error in the population dynamics. Canadian Journal of Fisheries and Aquatic Sciences 60 (2003), 1217-1228.

[79] Punt, A. E., AND Hilborn, R. Fisheries stock assessment and decision analysis: the Bayesian approach. Reviews in Fish Biology and Fisheries 7 (1997), 35-63.

[80] QUinN, T. J. I. Ruminations on the development and future of population dynamics models in fisheries. Natural Resource Modeling 16, 4 (1992), 341-392. http: //www. iattc.org/PDFFiles2/ Bulletins/IATTC-Bulletin-Vol-1-No-2.pdf.

[81] Quinn II, T. J., AND DERISON, R. B. Quantitative Fish Dynamics. Oxford University Press, New York, 1999.

[82] Robert, M., FAraj, A., McAllister, M. K., AND Rivot, E. Bayesian state-space modelling of the De Lury depletion model: strengths and limitations of the method, and application to the moroccan octopus fishery. ICES Journal of Marine Science 67 (2010), 12721290.

[83] Rose, G. A. Reconciling overfishing and limate change with stock dynamics of Atlantic Cod (Gadus morhua) over 500 years). Canadian Journal of Fisheries and Aquatic Sciences 61 (2004), 1553-1557.

[84] SCHAEFER, M. B. Some aspects of the dynamcis of populations important to the management of the commercial marine fisheries. Bulletin of the Inter-American Tropical Tuna Commission 1, 2 (2003), 27-56.

[85] Smith, D., Punt, A. E., Dowling, N., Smith, A., TuCK, G., AND KNUCKEY, I. Reconciling approaches to the assessment and management of data-poor species and fisheries with Australia's harvest 
strategy policy. Marine and Coastal Fisheries: Dynamcis, Management and Ecosystem Science 1 (2003), 244-254. doi. 10.1577/C08-041.1.

[86] Smith, M. T., AND AdDison, J. T. Models for stock assessment of crustacean fisheries. Fisheries Research 65 (2003), 231-256.

[87] Stevens, D., Smith, M. H., Grimes, P., Devine, J., Sutton, C., MaCGibBon, D., AND MaOlagain, C. O. Age, growth, and maturity of four New Zealand rattail species. New Zealand Aquatic Environment and Biodiversity Report No. 59. (2010), 39p. http://fs.fish. govt.nz/Doc/23019/AEBR_59.pdf.ashx.

[88] Stevens, D. W., AND DunN, M. R. Different food preferences in four sympatric deep-sea Macrourid fishes. Marine Biology 158 (2011), 59-72. doi: 10.1007/s00227-010-1542-1.

[89] Stevens, D. W., O'Driscoll, R. L., DunN, M. R. AND Ballara, S. L., AND HORN, P. Trawl survey of hoki and middle depth species on the Chatham Rise, January 2011 (TAN1101). New Zealand Fisheries Assessment Report 2012/10. (2012), 98p. http://docs . niwa. co.nz/library/public/FAR2012-10.pdf.

[90] Sturts, S., Ligges, U., AND Gelman, A. R2WinBUGS: A package for running WinBUGS from R. Journal of Statistical Software 12, 3 (2005), 1-16. http: / / www. stat. columbia.edu/ gelman/ research/published/JSS_R2WinBUGS.pdf.

[91] Williams, E. H., AND Prager, M. Comparison of equilibrium and nonequilibriaum estimators for the generalized production model. Canadian Journal of Fisheries and Aquatic Science 59 (2002), 1533-1552.

[92] WWW.MANCHEMAREE.FR. Fishing Methods: Trawling. April 2013. http://www.manchemaree.fr/eng/coastal-fishing/ fishing-methods/.

[93] ZhOU, S., Punt, A. E., DENG, R., AND Bishop, J. Estimating multifleet catchability coefficients and natural mortality from fishery catch and effort data: comparison of Bayesian state-space and observation error models. Canadian Journal of Fisheries and Aquatic Sciences 68 (2011), 1171-1181. 
Appendices 



\section{Appendix A}

\begin{tabular}{|c|c|c|c|c|c|c|}
\hline \multicolumn{7}{|c|}{$\begin{array}{l}\text { Summary of rattail species described in New Zealand Fishes, Volume I: } \\
\text { A field guide to common species caught by bottom and midwater trawling. [1] }\end{array}$} \\
\hline Common name & Genus and species & $\begin{array}{l}\text { MFish } \\
\text { reporting } \\
\text { code }\end{array}$ & $\begin{array}{c}\text { Mfish } \\
\text { research code }\end{array}$ & Size, $T L(\mathrm{~cm})$ & $\begin{array}{l}\text { Depth range } \\
\quad(\mathrm{m})\end{array}$ & Biology and ecology \\
\hline Codheaded rattail & Bathygadus cottoides & BAC & BAC & 30 & $>1100$ & Largely unknown. Probably demersal. \\
\hline Globosehead rattail & Cetonurus crassiceps & RAT & CCR & 44 & 900 to 1400 & Unknown. Probably demersal. \\
\hline Spotty faced rattail & Coelorinchus acanthiger & RAT & СTH & 50 & 800 to 1300 & Unknown. Probably demersal. \\
\hline Oblique banded rattail* & Coelorinchus aspercephalus & RAT & CAS & 56 & 30 to 600 & Demersal. \\
\hline Two saddle rattail* & Coelorinchus biclinozonalis & RAT & $\mathrm{CBI}$ & 62 & 5 to 500 & Demersal. \\
\hline Bollon's rattail* & Coelorinchus bollonsi & $\mathrm{CBO}$ & $\mathrm{CBO}$ & 71 & 300 to 700 & Largely unknown. Probably demersal. \\
\hline Black lip rattail & Coelorinchus celaenostomus & RAT & CEX & 83 & 600 to 1000 & Unknown. Demersal. \\
\hline Cook's rattail* & Coelorinchus cookianus & RAT & $\mathrm{CCO}$ & 30 & 500 to 800 & Unknown. Demersal. \\
\hline Banded rattail & Coelorinchus fasciatus & CFA & CFA & 40 & 500 to 1000 & Unknown. Demersal. \\
\hline Horrible rattail & Coelorinchus horribilis & RAT & $\mathrm{CXH}$ & 35 & 900 to 1200 & Unknown. Demersal. \\
\hline Notable rattail & Coelorinchus innotabilis & RAT & $\mathrm{CIN}$ & 41 & 500 to 1100 & Unknown. Demersal. \\
\hline Kaiyomaru rattail & Coelorinchus kaiyomaru & RAT & CKA & 45 & 800 to 120 & Unknown. Demersal. \\
\hline Mahia rattail & Coelorinchus matamu & CMA & CMA & 86 & 600 to 1000 & Unknown. Demersal. \\
\hline Dark banded rattail & Coelorinchus maurofasciatus & RAT & $\operatorname{CDX}$ & 50 & 300 to 800 & Unknown. Demersal. \\
\hline Upturned snout rattail & Coelorinchus mycterismus & RAT & $\mathrm{CJX}$ & 50 & 800 to 1200 & Unknown. Demersal. \\
\hline Oliver's rattail* & Coelorinchus oliverianus & $\mathrm{COL}$ & $\mathrm{COL}$ & 44 & 600 to 1000 & Unknown. Demersal. \\
\hline Small banded rattail & Coelorinchus parvifasciatus & RAT & $\operatorname{cCX}$ & 30 & 300 to 800 & Unknown. Demersal. \\
\hline Supanose rattail & Coelorinchus supernasutus & RAT & CFX & 65 & 500 to 900 & Unknown. Demersal. \\
\hline Roughhead rattail & Coelorinchus trachycarus & RAT & $\mathrm{CHY}$ & 56 & 800 to 1300 & Unknown. Demersal. \\
\hline Humpback rattail & Coryphaenoides dossenus & RAT & CBA & 85 & 900 to 1200 & Largely unknown. Demersal. \\
\hline McMillan's rattail & Coryphaenoides mcmillani & RAT & $\mathrm{CMX}$ & 39 & 900 to 1500 & Largely unknown. Demersal. \\
\hline Murray's rattail & Coryphaenoides murrayi & RAT & $\mathrm{CMU}$ & 81 & 700 to $>2300$ & Demersal. \\
\hline Serrulate rattail & Coryphaenoides serrulatus & RAT & CSE & 51 & 600 to 1200 & Largely unknown. Demersal. \\
\hline Striate rattail & Coryphaenoides striaturus & RAT & CTR & 55 & 800 to $>2000$ & Demersal. \\
\hline Four-rayed rattail & Coryphaenoides subserrulatus & RAT & CSU & 45 & 700 to 1200 & Largely unknown. Demersal. \\
\hline Filamentous rattail & Gadamus aoteanus & RAT & GAO & 50 & 1000 to 1400 & Unknown. Probably demersal. \\
\hline Pineapple rattail & Idiolophorhynchus andriashevi & RAT & PIN & 30 & 1000 to 1580 & Unknown. Demersal. \\
\hline Bulbous rattail & Kuronesumia bubonis & RAT & NBU & 80 & 500 to 1100 & Demersal. \\
\hline Kuronezumia leonis & Kuronezumia leonis & RAT & NPU & 52 & 700 to 1200 & Demersal. \\
\hline Javelinfish & Lepidorhynchus denticulatus & JAV & JAV & 72 & 250 to 1200 & Probably demersal. \\
\hline Blackspot rattail & Lucigadus nigromaculatus & RAT & $\mathrm{VNI}$ & 43 & 400 to 800 & Demersal. \\
\hline Ridge scaled rattail & Macrourus carinatus & MCA & MCA & 105 & 400 to 1500 & Demersal. \\
\hline Smooth headed rattail & Malacocephalus laevis & RAT & MLA & 65 & 200 to 1000 & Demersal. \\
\hline Black Javelinfish & Mesobius antipodum & RAT & BJA & 77 & 700 to 1300 & Largely unknown. \\
\hline Nezumia namatahi & Nezumia namatahi & RAT & NNA & 40 & 700 to 1200 & Demersal. \\
\hline Odontomacrurus murrayi & Odontomacrurus murrayi & RAT & $\mathrm{OMU}$ & 63 & $>800$ & Largely unknown. Possibly midwater. \\
\hline Velvet rattail & Trachonurus gagates & RAT & TRX & 48 & 435 to 1240 & Demersal. \\
\hline White rattail* & Trachyrincus aphyodes & WHX & WHX & 96 & 737 to 1140 & Demersal. \\
\hline Unicorn rattail & Trachyrincus longirostris & WHR & WHR & 50 & 1030 to 1400 & Demersal. \\
\hline
\end{tabular}




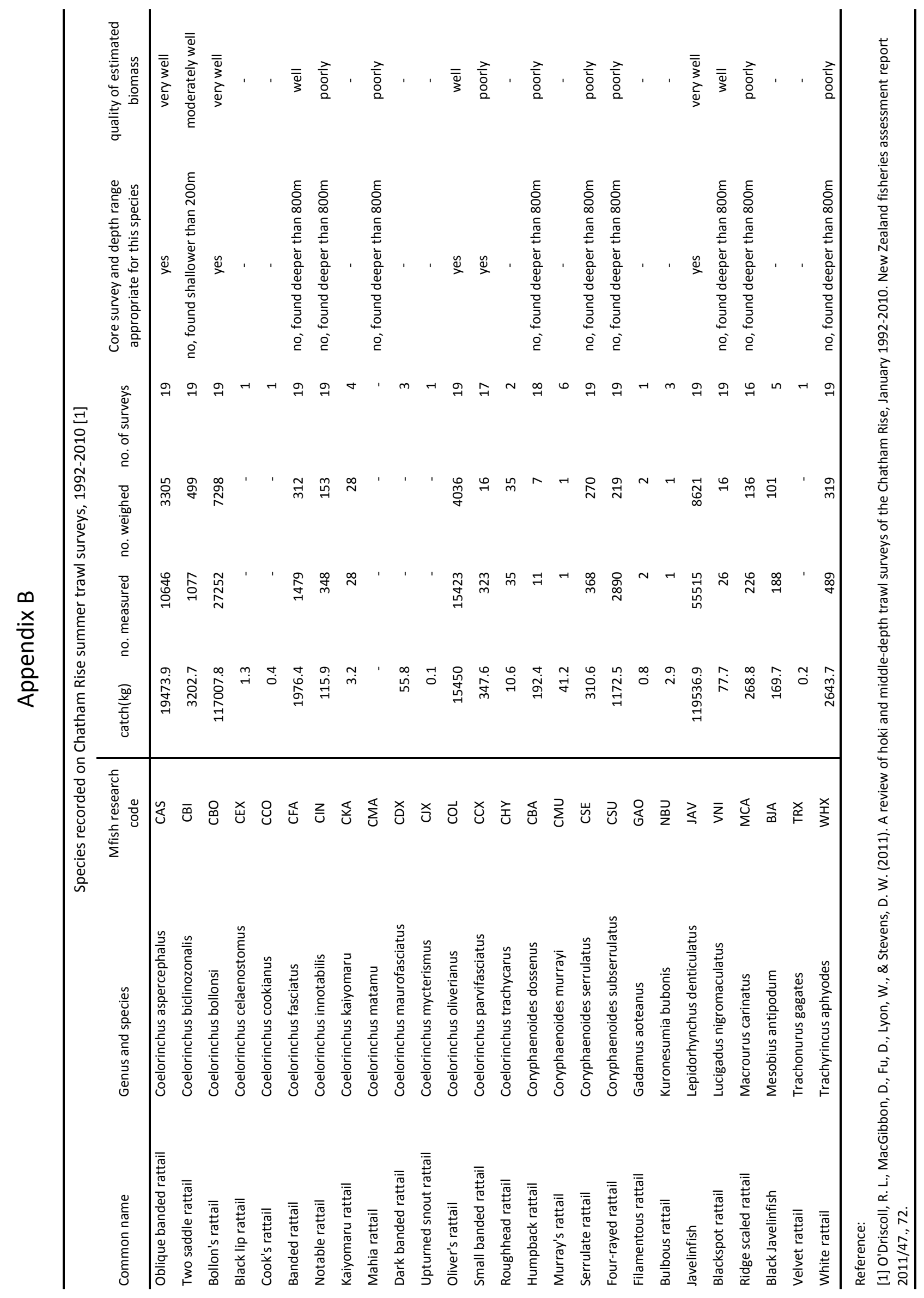




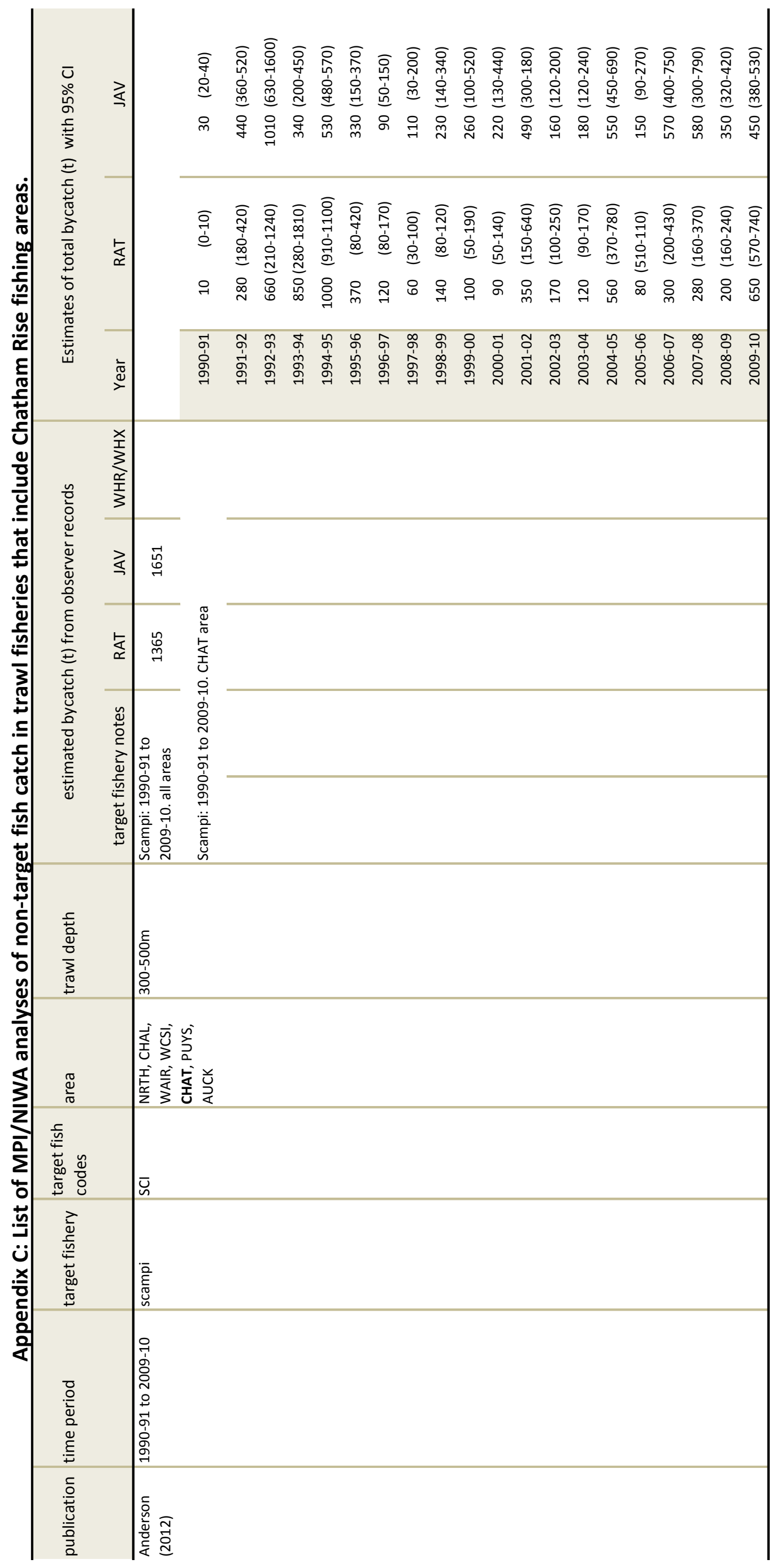




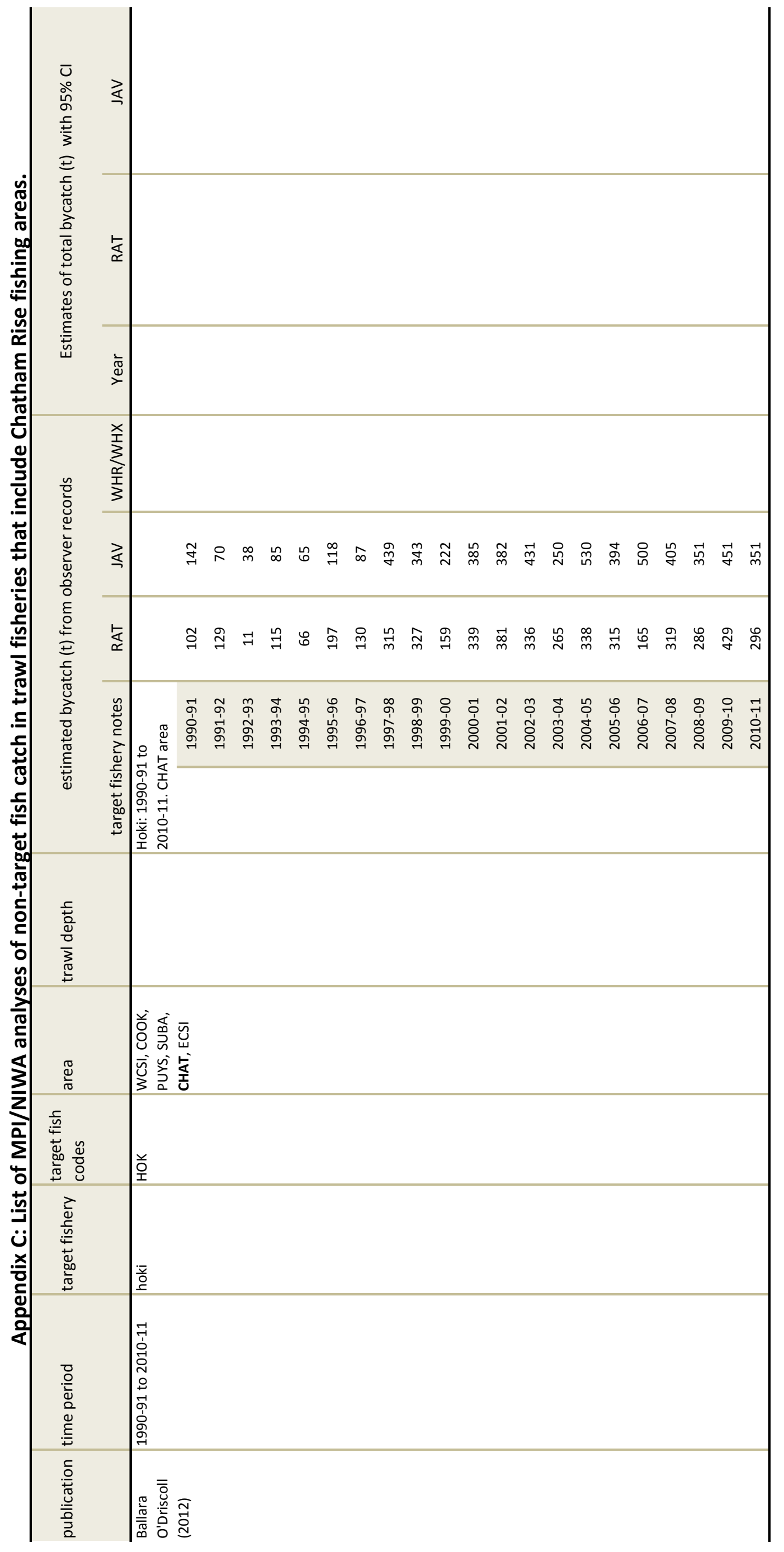




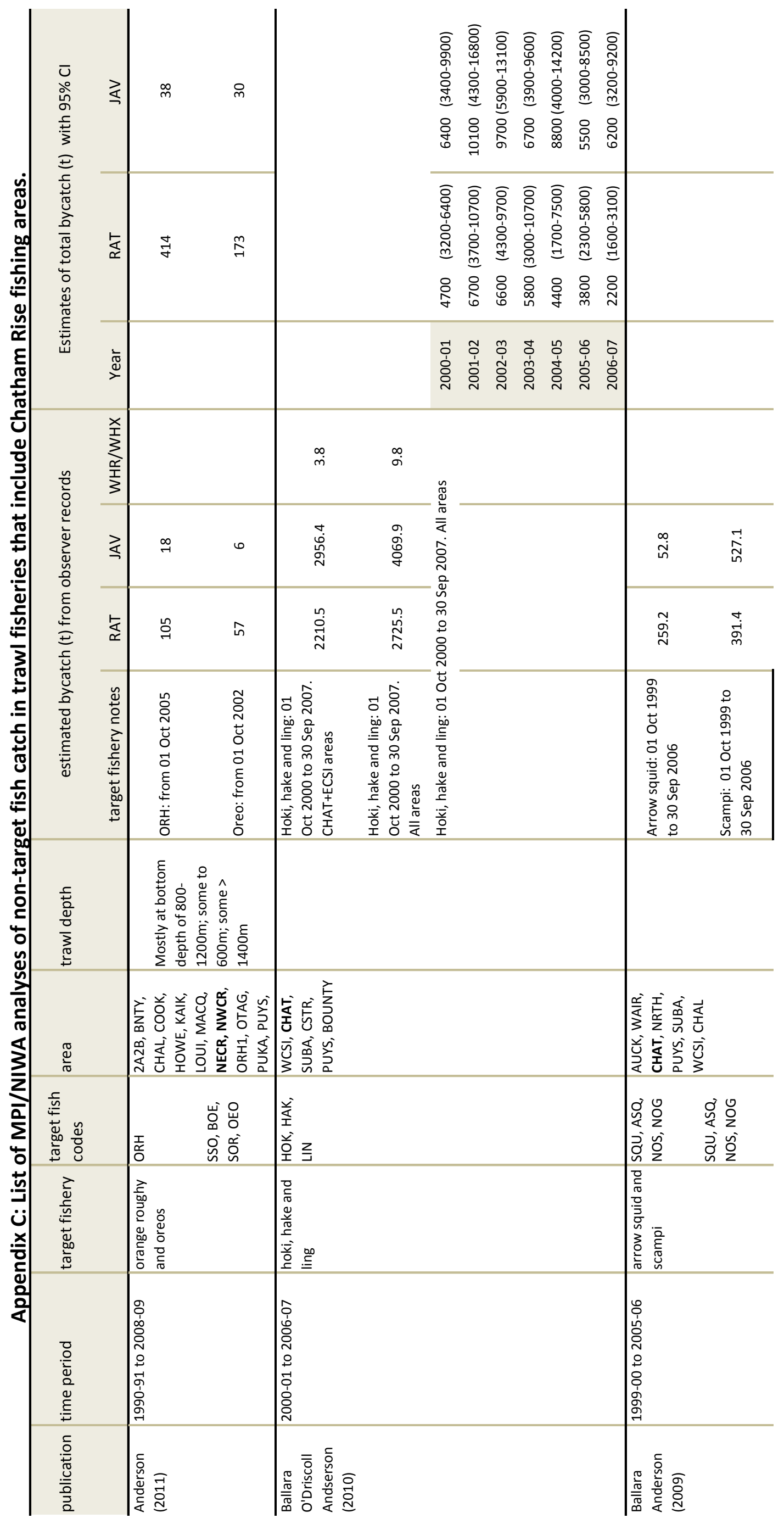




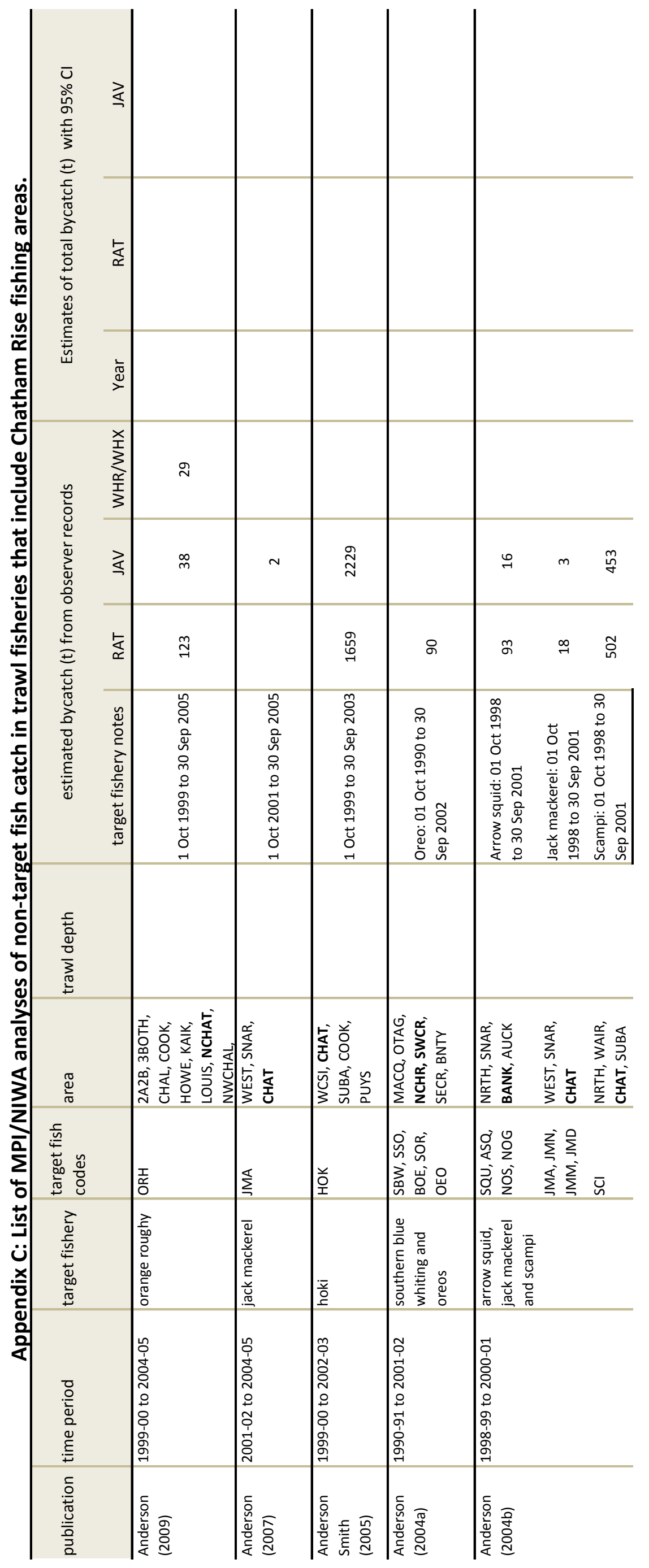




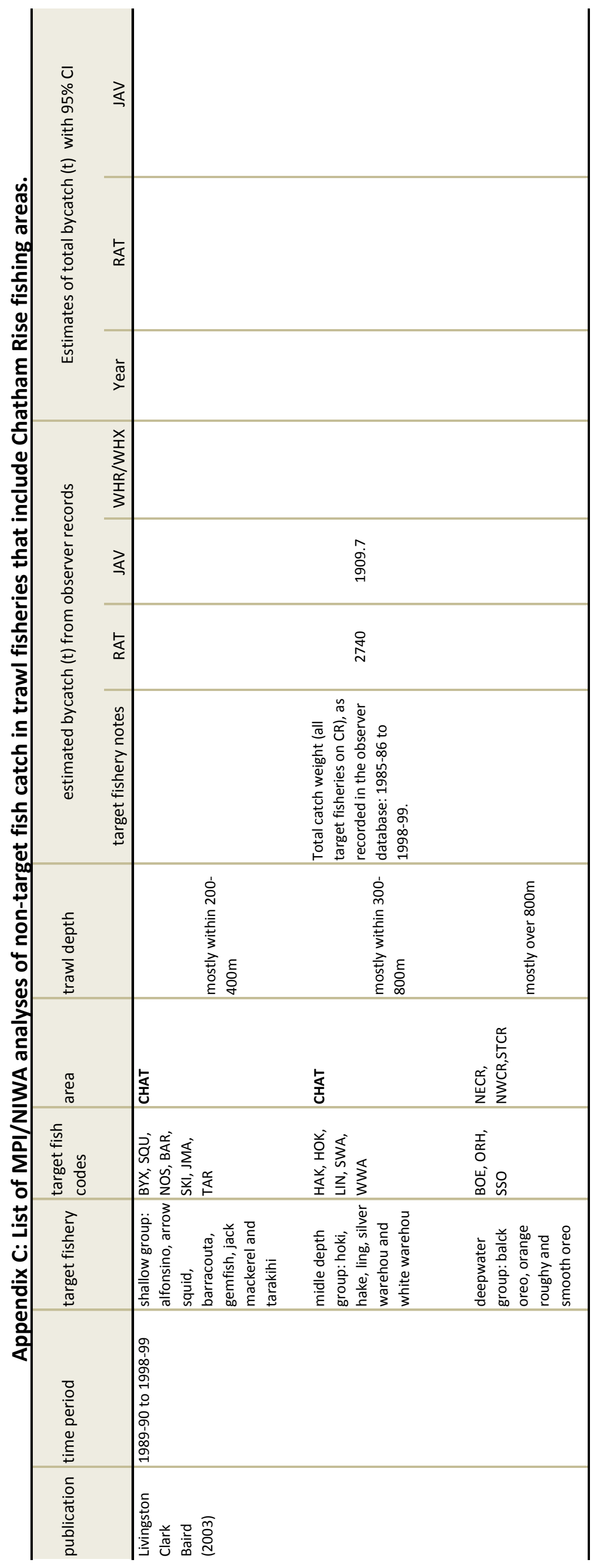




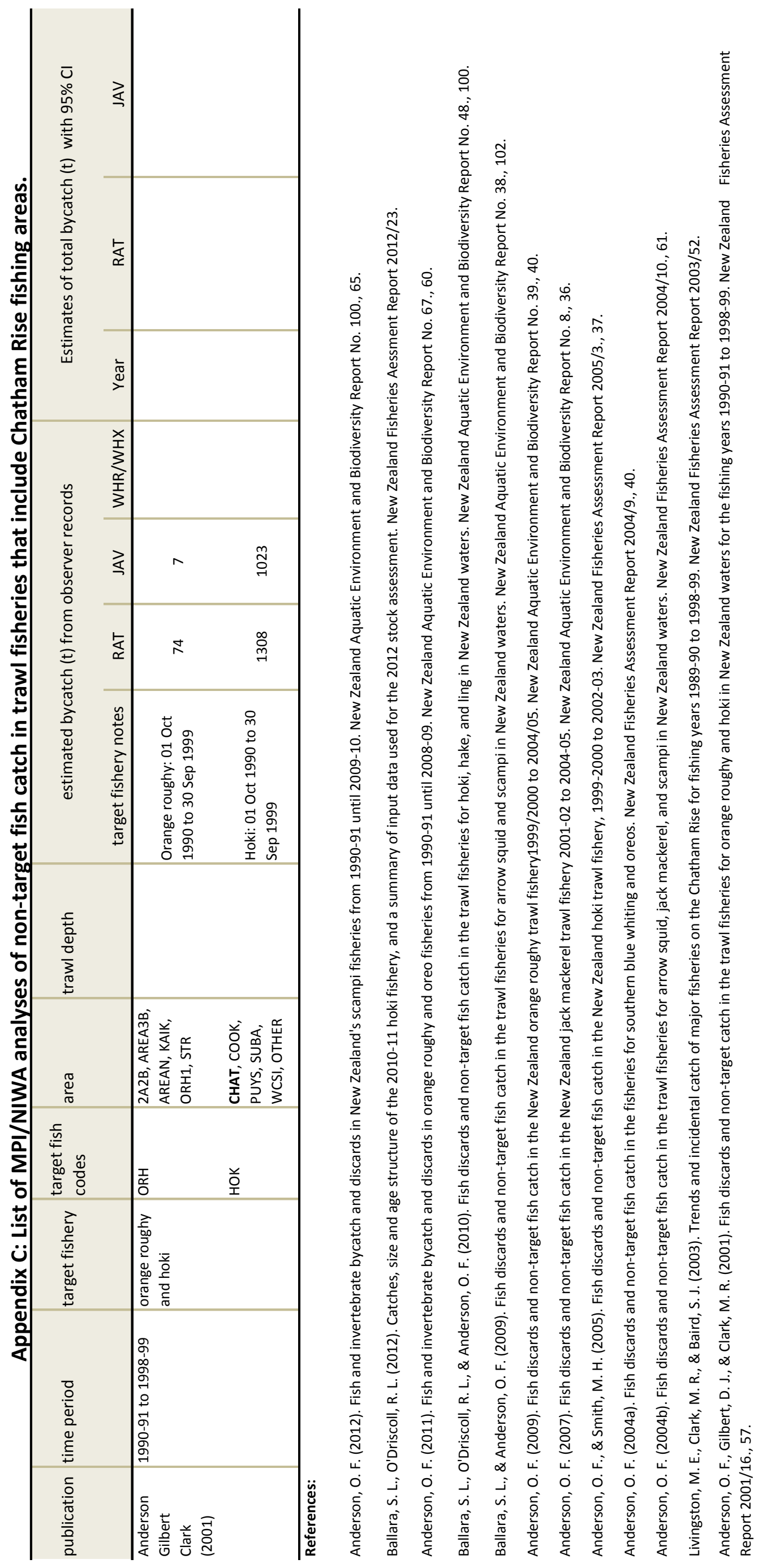


Appendix D:

Map, core survey strata

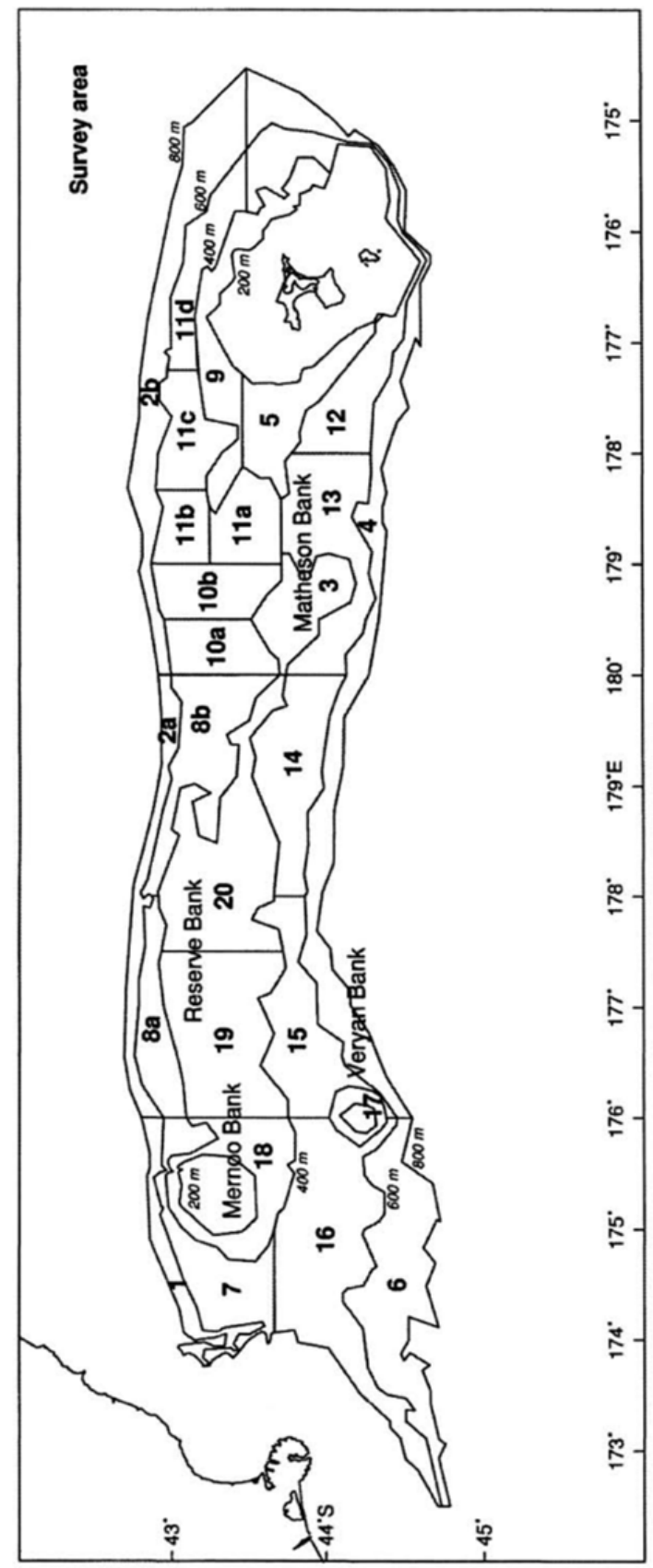

Figure 30: Core strata of the Chatham Rise trawl survey TAN1001. 


\begin{tabular}{|c|c|c|c|c|c|c|c|c|c|c|c|}
\hline $\begin{array}{r}00 \\
(600-8\end{array}$ & $\begin{array}{l}1 \\
00 \mathrm{~m})\end{array}$ & $\begin{array}{r}002 \\
(600-8\end{array}$ & $\begin{array}{l}\mathrm{A} \\
00 \mathrm{~m})\end{array}$ & $\begin{array}{r}00 \\
(200-4\end{array}$ & $\begin{array}{l}3 \\
00 \mathrm{~m})\end{array}$ & $\begin{array}{r}000 \\
(600-8\end{array}$ & $\begin{array}{l}4 \\
00 \mathrm{~m})\end{array}$ & $\begin{array}{r}00 \\
(200-4\end{array}$ & $\begin{array}{l}55 \\
00 \mathrm{~m})\end{array}$ & $\begin{array}{r}00 \\
(600-8\end{array}$ & $\begin{array}{l}66 \\
00 \mathrm{~m})\end{array}$ \\
\hline long & lat & long & lat & long & lat & long & lat & long & lat & long & lat \\
\hline 175.125 & -42.9233 & 180 & -43 & 181.0983 & -43.7167 & 176 & -44.45 & 182.715 & -43.47 & 176 & -44.5417 \\
\hline 174.915 & -42.9817 & 179.8883 & -43 & 181.0983 & -43.775 & 176.2317 & -44.3367 & 182.6267 & -43.5367 & 175.605 & -44.4767 \\
\hline 174.525 & -43.0883 & 179.7567 & -43.0717 & 180.9183 & -43.84 & 176.4733 & -44.1233 & 182.6367 & -43.6567 & 175.4067 & -44.6717 \\
\hline 174.29 & -43.1667 & 179.335 & -43.05 & 180.9383 & -43.915 & 176.5567 & -44.105 & 182.6233 & -43.6983 & 175.07 & -44.7233 \\
\hline 174.0817 & -43.21 & 179.1717 & -42.9817 & 181.07 & -43.945 & 176.6817 & -44.1133 & 182.6467 & -43.8283 & 175.0833 & -44.6983 \\
\hline 174.075 & -43.305 & 179.0617 & -43.005 & 181.0983 & -44.0233 & 176.845 & -43.9483 & 182.6683 & -43.845 & 174.8333 & -44.6117 \\
\hline 174.1067 & -43.3483 & 178.7633 & -42.9433 & 181.045 & -44.1567 & 177.2183 & -43.9417 & 182.715 & -43.8483 & 174.6967 & -44.6883 \\
\hline 174.16 & -43.59 & 178.1517 & -42.8467 & 180.83 & -44.2 & 177.4117 & -43.8233 & 182.7633 & -43.865 & 174.4883 & -44.63 \\
\hline 174.125 & -43.605 & 178.025 & -42.7983 & 180.6583 & -44.1633 & 177.6533 & -43.8783 & 182.785 & -43.93 & 174.1133 & -44.7117 \\
\hline 174.0467 & -43.505 & 177.9767 & -42.8167 & 180.5 & -43.96 & 178 & -43.8667 & 183.005 & -44.095 & 174.2117 & -44.5167 \\
\hline 174.0533 & -43.44 & 178.0183 & -42.8517 & 180.3583 & -43.9583 & 178.045 & -43.885 & 183.1267 & -44.21 & 173.9967 & -44.5167 \\
\hline 173.96 & -43.4083 & 178 & -42.8683 & 180.0917 & -43.7583 & 178.195 & -43.865 & 183.1917 & -44.3183 & 173.875 & -44.47 \\
\hline 173.9083 & -43.435 & 177.975 & -42.8817 & 180 & -43.7467 & 178.4667 & -43.89 & 183.2617 & -44.3433 & 173.8167 & -44.49 \\
\hline 173.9 & -43.405 & 177.895 & -42.86 & 180 & -43.7083 & 178.76 & -43.885 & 183.2567 & -44.3767 & 173.8883 & -44.525 \\
\hline 173.925 & -43.3933 & 177.9067 & -42.8167 & 180.1433 & -43.6983 & 178.9817 & -43.9933 & 183.1767 & -44.3267 & 173.775 & -44.65 \\
\hline 173.9117 & -43.22 & 177.5583 & -42.8283 & 180.2133 & -43.64 & 179.1233 & -43.9767 & 183.095 & -44.325 & 173.73 & -44.6033 \\
\hline 173.8333 & -43.2083 & 177.2333 & -42.7833 & 180.5 & -43.52 & 179.6233 & -44.0283 & 182.47 & -43.9667 & 173.5467 & -44.655 \\
\hline 173.7867 & -43.2517 & 177 & -42.7833 & 180.7183 & -43.6233 & 179.87 & -44.0917 & 182.39 & -43.8833 & 173.4183 & -44.6583 \\
\hline 173.775 & -43.235 & 176.5667 & -42.7583 & 180.8483 & -43.7167 & 180 & -44.1367 & 182.1833 & -43.8383 & 173.1133 & -44.7117 \\
\hline 173.7633 & -43.2333 & 176.2917 & -42.805 & 181 & -43.7167 & 180.1817 & -44.13 & 182.11 & -43.8467 & 173.0633 & -44.7533 \\
\hline 173.7617 & -43.2084 & 176 & -42.95 & 181.0983 & -43.7167 & 180.3767 & -44.2433 & 182 & -43.7867 & 172.9367 & -44.71 \\
\hline 173.7617 & -43.2083 & 176 & -42.8 & & & 180.5033 & -44.26 & 181.9317 & -43.7433 & 172.5 & -44.7833 \\
\hline 173.7817 & -43.2067 & 176.255 & -42.7283 & & & 180.6917 & -44.3233 & 181.64 & -43.7733 & 172.5 & -44.7283 \\
\hline 173.8467 & -43.1633 & 176.55 & -42.6917 & & & 180.9167 & -44.28 & 181.5867 & -43.7167 & 173.1083 & -44.63 \\
\hline 173.88 & -43.115 & 177.1667 & -42.7 & & & 181.115 & -44.3017 & 181.5902 & -43.7155 & 173.2717 & -44.5 \\
\hline 173.86 & -43.08 & 178.1033 & -42.7517 & & & 181.4283 & -44.175 & 181.7783 & -43.65 & 173.3717 & -44.5 \\
\hline 173.8917 & -43.07 & 178.7867 & -42.8833 & & & 181.5617 & -44.2083 & 181.7767 & -43.585 & 173.3667 & -44.5583 \\
\hline 173.93 & -43.0433 & 179.1967 & -42.94 & & & 181.5517 & -44.2783 & 181.8783 & -43.47 & 173.4783 & -44.5 \\
\hline 173.9517 & -43.0567 & 179.6533 & -42.9367 & & & 181.81 & -44.2967 & 182.715 & -43.47 & 173.505 & -44.385 \\
\hline 173.9767 & -43.2183 & 180 & -42.915 & & & 182 & -44.2917 & & & 173.5667 & -44.3267 \\
\hline 173.9433 & -43.2767 & 180 & -43 & & & 182.455 & -44.3133 & & & 173.75 & -44.265 \\
\hline 173.9433 & -43.3467 & & & & & 182.7217 & -44.4183 & & & 174.105 & -44.235 \\
\hline 174.0533 & -43.3583 & 002 & & & & 182.9183 & -44.4367 & & & 174.3417 & -44.1067 \\
\hline 174.0183 & -43.2817 & $(600-8$ & $00 \mathrm{~m})$ & & & 183.0383 & -44.5017 & & & 174.42 & -44.1067 \\
\hline 174.01 & -43.205 & long & lat & & & 183.365 & -44.52 & & & 174.4633 & -44.1367 \\
\hline 173.9917 & -43.1533 & 185.4683 & -43.5 & & & 183.7417 & -44.6483 & & & 174.4933 & -44.24 \\
\hline 174.0617 & -43.0817 & 184.7417 & -43.5 & & & 183.82 & -44.615 & & & 174.6333 & -44.2883 \\
\hline 174.2767 & -43.0517 & 184.74 & -43.5 & & & 183.9883 & -44.5 & & & 174.6667 & -44.32 \\
\hline 174.9 & -42.8583 & 184.3167 & -43.265 & & & 184.1083 & -44.465 & & & 174.955 & -44.23 \\
\hline 175.4683 & -42.8383 & 184.1817 & -43.2417 & & & 184.37 & -44.4817 & & & 175 & -44.2433 \\
\hline 175.5433 & -42.8567 & 184.0533 & -43.1033 & & & 184.6117 & -44.4167 & & & 175.0683 & -44.3317 \\
\hline 175.6783 & -42.8583 & 183.7183 & -43.0867 & & & 184.795 & -44.3083 & & & 175.1333 & -44.3667 \\
\hline 176 & -42.8 & 183.4 & -43 & & & 184.8083 & -44.15 & & & 175.3333 & -44.3667 \\
\hline 176 & -42.95 & 182.94 & -43.0067 & & & 184.8983 & -43.9283 & & & 175.3933 & -44.2917 \\
\hline 175.6783 & -42.94 & 182.9383 & -42.9767 & & & 184.9267 & -43.75 & & & 175.4783 & -44.245 \\
\hline 175.5717 & -42.9367 & 182.9117 & -42.965 & & & 184.975 & -43.6667 & & & 175.6533 & -44.2367 \\
\hline 175.5267 & -42.9667 & 182.8733 & -42.9933 & & & 184.9067 & -43.5983 & & & 175.7167 & -44.32 \\
\hline 175.5033 & -42.92 & 182.685 & -42.9767 & & & 184.74 & -43.5 & & & 175.9333 & -44.44 \\
\hline 175.46 & -42.8833 & 182.6333 & -42.9283 & & & 185.4683 & -43.5 & & & 176 & -44.45 \\
\hline 175.2033 & -42.9317 & 182.4817 & -42.9233 & & & 185.2467 & -43.8533 & & & 176 & -44.5417 \\
\hline 175.185 & -42.9467 & 182.3283 & -43.0083 & & & 185 & -44.0717 & & & & \\
\hline 175.125 & -42.9233 & 182 & -42.9167 & & & 184.86 & -44.16 & & & & \\
\hline & & 181.6677 & -42.9001 & & & 184.9183 & -44.2 & & & & \\
\hline & & 181.6667 & -42.9 & & & 184.78 & -44.3467 & & & & \\
\hline & & 181.5167 & -42.95 & & & 184.6067 & -44.4383 & & & & \\
\hline & & 181 & -42.8667 & & & 184.375 & -44.52 & & & & \\
\hline & & 180.5867 & -42.92 & & & 184.005 & -44.51 & & & & \\
\hline & & 180.5005 & -42.9536 & & & 183.79 & -44.6717 & & & & \\
\hline & & 180 & -43 & & & 183.7183 & -44.6733 & & & & \\
\hline & & 180 & -42.915 & & & 183.5333 & -44.6067 & & & & \\
\hline & & 180.5183 & -42.8583 & & & 182.9667 & -44.6067 & & & & \\
\hline & & 180.9367 & -42.785 & & & 183.0433 & -44.5783 & & & & \\
\hline & & 181.0533 & -42.8083 & & & 182.655 & -44.4817 & & & & \\
\hline & & 181.2833 & -42.8083 & & & 182.4167 & -44.53 & & & & \\
\hline & & 181.6833 & -42.7167 & & & 182.2917 & -44.4583 & & & & \\
\hline & & 182.32 & -42.79 & & & 181.8683 & -44.36 & & & & \\
\hline & & 182.6667 & -42.7933 & & & 181.6467 & -44.3917 & & & & \\
\hline & & 183.205 & -42.8483 & & & 181.515 & -44.365 & & & & \\
\hline & & 184.2583 & -43.0083 & & & 181.3767 & -44.3667 & & & & \\
\hline & & 184.5983 & -43.0833 & & & 181.3033 & -44.39 & & & & \\
\hline & & 184.94 & -43.095 & & & 180.4983 & -44.34 & & & & \\
\hline & & 184.9917 & -43.155 & & & 180 & -44.2783 & & & & \\
\hline & & 185.4683 & -43.5 & & & 179.62 & -44.1233 & & & & \\
\hline & & & & & & 179.175 & -44.115 & & & & \\
\hline & & & & & & 178.7883 & -44.0567 & & & & \\
\hline & & & & & & 178.2983 & -44.0533 & & & & \\
\hline & & & & & & 178.0383 & -43.98 & & & & \\
\hline & & & & & & 177.6867 & -43.945 & & & & \\
\hline & & & & & & 177.525 & -44.0317 & & & & \\
\hline & & & & & & 177.34 & -44.0433 & & & & \\
\hline & & & & & & 177.1483 & -44.16 & & & & \\
\hline & & & & & & 177.0267 & -44.1567 & & & & \\
\hline & & & & & & 176.775 & -44.2717 & & & & \\
\hline & & & & & & 176.375 & -44.3783 & & & & \\
\hline & & & & & & 176 & -44.5417 & & & & \\
\hline
\end{tabular}




\begin{tabular}{|c|c|c|c|c|c|c|c|c|c|c|c|c|c|}
\hline \multicolumn{2}{|c|}{$\begin{array}{c}008 \mathrm{~A} \\
(400-600 \mathrm{~m})\end{array}$} & \multicolumn{2}{|c|}{$\begin{array}{c}0009 \\
(200-400 \mathrm{~m})\end{array}$} & \multicolumn{2}{|c|}{$\begin{array}{c}010 \mathrm{~A} \\
(400-600 \mathrm{~m})\end{array}$} & \multicolumn{2}{|c|}{$\begin{array}{c}011 \mathrm{~A} \\
(400-600 \mathrm{~m})\end{array}$} & \multicolumn{2}{|c|}{$\begin{array}{c}0012 \\
(400-600 \mathrm{~m})\end{array}$} & \multicolumn{2}{|c|}{$\begin{array}{c}0013 \\
(400-600 \mathrm{~m})\end{array}$} & $\begin{array}{r}00 \\
(400-6\end{array}$ & $\begin{array}{l}14 \\
00 \mathrm{~m})\end{array}$ \\
\hline long & lat & long & lat & long & lat & long & lat & long & lat & long & lat & long & lat \\
\hline 177.5 & -42.9333 & 184.525 & -44.0417 & 180.5 & -43.52 & 181.525 & -43.255 & 184.74 & -43.5 & 182 & -44.2917 & 180 & -43.7467 \\
\hline 177.4 & -42.9167 & 184.3867 & -44.0017 & 180.2133 & -43.64 & 181.4583 & -43.2967 & 184.9067 & -43.5983 & 181.81 & -44.2967 & 180 & -43.7483 \\
\hline 177 & -42.95 & 184.42 & -43.9283 & 180.1433 & -43.6983 & 181.8783 & -43.47 & 184.975 & -43.6667 & 181.5517 & -44.2783 & 180 & -44.1367 \\
\hline 176.4917 & -43.0517 & 184.3717 & -43.91 & 180 & -43.7083 & 181.7767 & -43.585 & 184.9267 & -43.75 & 181.5617 & -44.2083 & 179.87 & -44.0917 \\
\hline 176.3217 & -43.08 & 184.3367 & -43.845 & 180 & -43 & 181.7783 & -43.65 & 184.8983 & -43.9283 & 181.4283 & -44.175 & 179.6233 & -44.0283 \\
\hline 176 & -43.1017 & 184.2367 & -43.8433 & 180.5005 & -42.9536 & 181.5902 & -43.7155 & 184.8083 & -44.15 & 181.115 & -44.3017 & 179.1233 & -43.9767 \\
\hline 176 & -42.95 & 184.2267 & -43.8067 & 180.5 & -43.52 & 181.5867 & -43.7167 & 184.795 & -44.3083 & 180.9167 & -44.28 & 178.9817 & -43.9933 \\
\hline 176.2917 & -42.805 & 184.2017 & -43.825 & & & 181.0983 & -43.7167 & 184.6117 & -44.4167 & 180.6917 & -44.3233 & 178.76 & -43.885 \\
\hline 176.5667 & -42.7583 & 184.155 & -43.785 & 01 & & 181 & -43.7167 & 184.37 & -44.4817 & 180.5033 & -44.26 & 178.4667 & -43.89 \\
\hline 177 & -42.7833 & 184.1117 & -43.6583 & $(400-6$ & 00m) & 181 & -43.255 & 184.1083 & -44.465 & 180.3767 & -44.2433 & 178.195 & -43.865 \\
\hline 177.2333 & -42.7833 & 184.0533 & -43.635 & long & lat & 181.525 & -43.255 & 183.9883 & -44.5 & 180.1817 & -44.13 & 178.045 & -43.885 \\
\hline 177.5583 & -42.8283 & 184.0033 & -43.6033 & 181 & -42.8667 & & & 183.82 & -44.615 & 180 & -44.1367 & 178 & -43.8667 \\
\hline 177.9067 & -42.8167 & 183.9283 & -43.605 & 181 & -43.7167 & 01 & & 183.7417 & -44.6483 & 180 & -43.7483 & 178 & -43.6667 \\
\hline 177.895 & -42.86 & 183.8417 & -43.5367 & 180.8483 & -43.7167 & $(400-6$ & 00m) & 183.365 & -44.52 & 180 & -43.7467 & 178.49 & -43.7 \\
\hline 177.975 & -42.8817 & 183.825 & -43.5083 & 180.7183 & -43.6233 & long & lat & 183.0383 & -44.5017 & 180.0917 & -43.7583 & 179.0817 & -43.5283 \\
\hline 178 & -42.8683 & 183.845 & -43.4467 & 180.5 & -43.52 & 181 & -43.255 & 182.9183 & -44.4367 & 180.3583 & -43.9583 & 179.2567 & -43.5517 \\
\hline 178 & -42.915 & 183.825 & -43.4183 & 180.5005 & -42.9536 & 181 & -42.8667 & 182.7217 & -44.4183 & 180.5 & -43.96 & 179.43 & -43.515 \\
\hline 177.8983 & -42.92 & 183.7867 & -43.405 & 180.5867 & -42.92 & 181.5167 & -42.95 & 182.455 & -44.3133 & 180.6583 & -44.1633 & 179.6783 & -43.6583 \\
\hline 177.7383 & -42.9667 & 183.5817 & -43.425 & 181 & -42.8667 & 181.6667 & -42.9 & 182 & -44.2917 & 180.83 & -44.2 & 179.72 & -43.7533 \\
\hline 177.5 & -42.9333 & 183.51 & -43.3867 & & & 181.6667 & -43.2367 & 182 & -43.7867 & 181.045 & -44.1567 & 179.8083 & -43.7267 \\
\hline & & 183.4383 & -43.33 & & & 181.525 & -43.255 & 182.11 & -43.8467 & 181.0983 & -44.0233 & 180 & -43.7467 \\
\hline 008 & & 183.345 & -43.3283 & & & 181 & -43.255 & 182.1833 & -43.8383 & 181.07 & -43.945 & & \\
\hline$(400-6$ & 00m) & 183.2267 & -43.24 & & & & & 182.39 & -43.8833 & 180.9383 & -43.915 & & \\
\hline long & lat & 182.795 & -43.4083 & & & 01 & & 182.47 & -43.9667 & 180.9183 & -43.84 & & \\
\hline 180 & -43 & 182.715 & -43.47 & & & $(400-6$ & 00m) & 183.095 & -44.325 & 181.0983 & -43.775 & & \\
\hline 180 & -43.7083 & 181.8783 & -43.47 & & & long & lat & 183.1767 & -44.3267 & 181.0983 & -43.7167 & & \\
\hline 179.79 & -43.5533 & 181.4583 & -43.2967 & & & 182.75 & -43.1833 & 183.2567 & -44.3767 & 181.5867 & -43.7167 & & \\
\hline 179.5883 & -43.5083 & 181.525 & -43.255 & & & 182.6667 & -43.1933 & 183.2333 & -44.4283 & 181.64 & -43.7733 & & \\
\hline 179.4583 & -43.3683 & 181.6667 & -43.2367 & & & 182.31 & -43.23 & 183.2467 & -44.4483 & 181.9317 & -43.7433 & & \\
\hline 179.3717 & -43.3933 & 181.7967 & -43.2367 & & & 182.25 & -43.4383 & 183.73 & -44.6067 & 182 & -43.7867 & & \\
\hline 179.3683 & -43.4433 & 182 & -43.3367 & & & 182.1267 & -43.4383 & 184.1217 & -44.39 & 182 & -44.2917 & & \\
\hline 179.0833 & -43.43 & 182.1267 & -43.4383 & & & 182 & -43.3367 & 184.275 & -44.4167 & & & & \\
\hline 178.89 & -43.3233 & 182.25 & -43.4383 & & & 181.7967 & -43.2367 & 184.57 & -44.3933 & & & & \\
\hline 178.4833 & -43.3 & 182.31 & -43.23 & & & 181.6667 & -43.2367 & 184.7683 & -44.3 & & & & \\
\hline 178.55 & -43.1833 & 182.6667 & -43.1933 & & & 181.6667 & -42.9 & 184.7883 & -44.095 & & & & \\
\hline 178.8583 & -43.1417 & 183.23 & -43.165 & & & 181.6677 & -42.9001 & 184.525 & -44.0417 & & & & \\
\hline 178.9333 & -43.2583 & 183.6567 & -43.2133 & & & 182 & -42.9167 & 184.6667 & -43.9333 & & & & \\
\hline 179.0167 & -43.1583 & 183.6867 & -43.2483 & & & 182.3283 & -43.0083 & 184.6667 & -43.8333 & & & & \\
\hline 178.9767 & -43.06 & 183.635 & -43.2733 & & & 182.4817 & -42.9233 & 184.4833 & -43.7833 & & & & \\
\hline 178.605 & -43.0067 & 183.6967 & -43.305 & & & 182.6333 & -42.9283 & 184.3817 & -43.73 & & & & \\
\hline 178.555 & -43.0517 & 184.1817 & -43.4267 & & & 182.685 & -42.9767 & 184.3317 & -43.7767 & & & & \\
\hline 178.0367 & -42.9117 & 184.1833 & -43.475 & & & 182.75 & -42.975 & 184.3 & -43.7433 & & & & \\
\hline 178 & -42.915 & 184.1766 & -43.5 & & & 182.75 & -43.1833 & 184.4 & -43.6183 & & & & \\
\hline 178 & -42.8683 & 184.1567 & -43.5733 & & & & & 184.3667 & -43.5617 & & & & \\
\hline 178.0183 & -42.8517 & 184.2067 & -43.5883 & & & 01 & & 184.2333 & -43.615 & & & & \\
\hline 177.9767 & -42.8167 & 184.2333 & -43.615 & & & $(400-6$ & $00 \mathrm{~m})$ & 184.2067 & -43.5883 & & & & \\
\hline 178.025 & -42.7983 & 184.3667 & -43.5617 & & & long & lat & 184.1567 & -43.5733 & & & & \\
\hline 178.1517 & -42.8467 & 184.4 & -43.6183 & & & 182.75 & -42.975 & 184.1766 & -43.5 & & & & \\
\hline 178.7633 & -42.9433 & 184.3 & -43.7433 & & & 182.8733 & -42.9933 & 184.1767 & -43.5 & & & & \\
\hline 179.0617 & -43.005 & 184.3317 & -43.7767 & & & 182.9117 & -42.965 & 184.74 & -43.5 & & & & \\
\hline 179.1717 & -42.9817 & 184.3817 & -43.73 & & & 182.9383 & -42.9767 & & & & & & \\
\hline 179.335 & -43.05 & 184.4833 & -43.7833 & & & 182.94 & -43.0067 & & & & & & \\
\hline 179.7567 & -43.0717 & 184.6667 & -43.8333 & & & 183.4 & -43 & & & & & & \\
\hline 179.8883 & -43 & 184.6667 & -43.9333 & & & 183.7183 & -43.0867 & & & & & & \\
\hline 180 & -43 & 184.525 & -44.0417 & & & 184.0533 & -43.1033 & & & & & & \\
\hline & & & & & & 184.1817 & -43.2417 & & & & & & \\
\hline & & & & & & 184.3167 & -43.265 & & & & & & \\
\hline & & & & & & 184.74 & -43.5 & & & & & & \\
\hline & & & & & & 184.1767 & -43.5 & & & & & & \\
\hline & & & & & & 184.1766 & -43.5 & & & & & & \\
\hline & & & & & & 184.1766 & -43.5 & & & & & & \\
\hline & & & & & & 184.1833 & -43.475 & & & & & & \\
\hline & & & & & & 184.1817 & -43.4267 & & & & & & \\
\hline & & & & & & 183.6967 & -43.305 & & & & & & \\
\hline & & & & & & 183.635 & -43.2733 & & & & & & \\
\hline & & & & & & 183.6867 & -43.2483 & & & & & & \\
\hline & & & & & & 183.6567 & -43.2133 & & & & & & \\
\hline & & & & & & 183.23 & -43.165 & & & & & & \\
\hline & & & & & & 182.75 & -43.1833 & & & & & & \\
\hline & & & & & & 182.75 & -42.975 & & & & & & \\
\hline
\end{tabular}


Appendix E: Core strata polygons (by longitude and latitude), Chatham Rise Survey TAN1001

\begin{tabular}{|c|c|c|c|c|c|c|c|c|c|c|c|}
\hline \multicolumn{2}{|c|}{$\begin{array}{c}0015 \\
(400-600 \mathrm{~m})\end{array}$} & \multicolumn{2}{|c|}{$\begin{array}{c}0016 \\
(400-600 \mathrm{~m})\end{array}$} & \multicolumn{2}{|c|}{$\begin{array}{c}017 \mathrm{~A} \\
(200-400 \mathrm{~m})\end{array}$} & \multicolumn{2}{|c|}{$\begin{array}{c}018 \mathrm{~A} \\
(200-400 \mathrm{~m})\end{array}$} & \multicolumn{2}{|c|}{$\begin{array}{c}0019 \\
(200-400 \mathrm{~m})\end{array}$} & \multicolumn{2}{|c|}{$\begin{array}{c}0020 \\
(200-400 \mathrm{~m})\end{array}$} \\
\hline long & lat & long & lat & long & lat & long & lat & long & lat & long & lat \\
\hline 176 & -44.45 & 172.5 & -44.7283 & 176.1033 & -44.3667 & 176 & -43.7583 & 177.355 & -43.7433 & 180 & -43.7467 \\
\hline 176 & -44.3783 & 172.5 & -44.7033 & 176 & -44.3783 & 175.8717 & -43.8033 & 177.1267 & -43.645 & 179.8083 & -43.7267 \\
\hline 176.1033 & -44.3667 & 172.8217 & -44.58 & 175.9583 & -44.38 & 175.6133 & -43.8033 & 177.0817 & -43.5633 & 179.72 & -43.7533 \\
\hline 176.2 & -44.3417 & 172.9267 & -44.5 & 175.8917 & -44.345 & 175.575 & -43.7567 & 176.7567 & -43.6683 & 179.6783 & -43.6583 \\
\hline 176.2417 & -44.3 & 173.1117 & -44.465 & 175.7417 & -44.2183 & 175.5 & -43.7983 & 176.4717 & -43.5783 & 179.43 & -43.515 \\
\hline 176.27 & -44.2383 & 173.2083 & -44.4 & 175.89 & -44.07 & 175.1883 & -43.73 & 176.165 & -43.755 & 179.2567 & -43.5517 \\
\hline 176.2867 & -44.1483 & 173.2717 & -44.3967 & 176 & -44.02 & 175.135 & -43.69 & 176 & -43.7583 & 179.0817 & -43.5283 \\
\hline 176.2383 & -44.0633 & 173.52 & -44.215 & 176.2383 & -44.0633 & 175 & -43.6667 & 176 & -43.1017 & 178.49 & -43.7 \\
\hline 176 & -44.02 & 173.9283 & -44.015 & 176.2867 & -44.1483 & 174.85 & -43.6317 & 176.3217 & -43.08 & 178 & -43.6667 \\
\hline 176 & -44.0183 & 173.9783 & -43.82 & 176.27 & -44.2383 & 174.705 & -43.545 & 176.4917 & -43.0517 & 177.955 & -43.6667 \\
\hline 176 & -43.7583 & 174.09 & -43.7983 & 176.2417 & -44.3 & 174.7267 & -43.37 & 177 & -42.95 & 177.8983 & -43.565 \\
\hline 176.165 & -43.755 & 174.065 & -43.6667 & 176.2 & -44.3417 & 174.8317 & -43.3033 & 177.4 & -42.9167 & 177.7883 & -43.5233 \\
\hline 176.4717 & -43.5783 & 174.0683 & -43.6667 & 176.1033 & -44.3667 & 174.895 & -43.1617 & 177.5 & -42.9333 & 177.705 & -43.6983 \\
\hline 176.7567 & -43.6683 & 175 & -43.6667 & & & 175.0183 & -43.0633 & 177.5 & -43.7247 & 177.5 & -43.7247 \\
\hline 177.0817 & -43.5633 & 175.135 & -43.69 & 01 & & 175.1933 & -42.9783 & 177.355 & -43.7433 & 177.5 & -42.9333 \\
\hline 177.1267 & -43.645 & 175.1883 & -43.73 & (excl):< & $200 \mathrm{~m}$ ) & 175.4733 & -42.9633 & & & 177.7383 & -42.9667 \\
\hline 177.355 & -43.7433 & 175.5 & -43.7983 & long & lat & 175.5067 & -42.9783 & & & 177.8983 & -42.92 \\
\hline 177.5 & -43.7247 & 175.575 & -43.7567 & 176.1317 & -44.185 & 175.5167 & -43.0117 & & & 178 & -42.915 \\
\hline 177.705 & -43.6983 & 175.6133 & -43.8033 & 176.0217 & -44.0917 & 175.5333 & -42.9933 & & & 178.0367 & -42.9117 \\
\hline 177.7883 & -43.5233 & 175.8717 & -43.8033 & 175.88 & -44.1883 & 175.6017 & -42.975 & & & 178.555 & -43.0517 \\
\hline 177.8983 & -43.565 & 176 & -43.7583 & 175.8633 & -44.2583 & 175.6567 & -42.9967 & & & 178.605 & -43.0067 \\
\hline 177.955 & -43.6667 & 176 & -44.0183 & 175.9317 & -44.3167 & 175.6783 & -43.1283 & & & 178.9767 & -43.06 \\
\hline 178 & -43.6667 & 176 & -44.02 & 176.08 & -44.3083 & 175.8133 & -43.3067 & & & 179.0167 & -43.1583 \\
\hline 178 & -43.8667 & 175.89 & -44.07 & 176.1183 & -44.2617 & 175.9533 & -43.23 & & & 178.9333 & -43.2583 \\
\hline 177.6533 & -43.8783 & 175.7417 & -44.2183 & 176.1317 & -44.185 & 175.9233 & -43.14 & & & 178.8583 & -43.1417 \\
\hline 177.4117 & -43.8233 & 175.8917 & -44.345 & & & 176 & -43.1017 & & & 178.55 & -43.1833 \\
\hline 177.2183 & -43.9417 & 175.9583 & -44.38 & & & 176 & -43.7583 & & & 178.4833 & -43.3 \\
\hline 176.845 & -43.9483 & 176 & -44.3783 & & & & & & & 178.89 & -43.3233 \\
\hline 176.6817 & -44.1133 & 176 & -44.45 & & & 01 & & & & 179.0833 & -43.43 \\
\hline 176.5567 & -44.105 & 175.9333 & -44.44 & & & (excl: $<$ & $200 \mathrm{~m}$ ) & & & 179.3683 & -43.4433 \\
\hline 176.4733 & -44.1233 & 175.7167 & -44.32 & & & long & lat & & & 179.3717 & -43.3933 \\
\hline 176.2317 & -44.3367 & 175.6533 & -44.2367 & & & 175.2317 & -43.0517 & & & 179.4583 & -43.3683 \\
\hline \multirow{23}{*}{176} & -44.45 & 175.4783 & -44.245 & & & 175.1933 & -43.0667 & & & 179.5883 & -43.5083 \\
\hline & & 175.3933 & -44.2917 & & & 174.945 & -43.2733 & & & 179.79 & -43.5533 \\
\hline & & 175.3333 & -44.3667 & & & 174.975 & -43.4583 & & & 180 & -43.7083 \\
\hline & & 175.1333 & -44.3667 & & & 175.1017 & -43.555 & & & 180 & -43.7467 \\
\hline & & 175.0683 & -44.3317 & & & 175.475 & -43.5433 & & & & \\
\hline & & 175 & -44.2433 & & & 175.595 & -43.4417 & & & & \\
\hline & & 174.955 & -44.23 & & & 175.6517 & -43.365 & & & & \\
\hline & & 174.6667 & -44.32 & & & 175.665 & -43.21 & & & & \\
\hline & & 174.6333 & -44.2883 & & & 175.6 & -43.0867 & & & & \\
\hline & & 174.4933 & -44.24 & & & 175.56 & -43.0433 & & & & \\
\hline & & 174.4633 & -44.1367 & & & 175.54 & -43.045 & & & & \\
\hline & & 174.42 & -44.1067 & & & 175.5333 & -43.0767 & & & & \\
\hline & & 174.3417 & -44.1067 & & & 175.5083 & -43.045 & & & & \\
\hline & & 174.105 & -44.235 & & & 175.3933 & -43.0667 & & & & \\
\hline & & 173.75 & -44.265 & & & 175.2317 & -43.0517 & & & & \\
\hline & & 173.5667 & -44.3267 & & & & & & & & \\
\hline & & 173.505 & -44.385 & & & & & & & & \\
\hline & & 173.4783 & -44.5 & & & & & & & & \\
\hline & & 173.3667 & -44.5583 & & & & & & & & \\
\hline & & 173.3717 & -44.5 & & & & & & & & \\
\hline & & 173.2717 & -44.5 & & & & & & & & \\
\hline & & 173.1083 & -44.63 & & & & & & & & \\
\hline & & 172.5 & -44.7283 & & & & & & & & \\
\hline
\end{tabular}




\section{Appendix F: Commercial effort (hours) by core stratum by year}

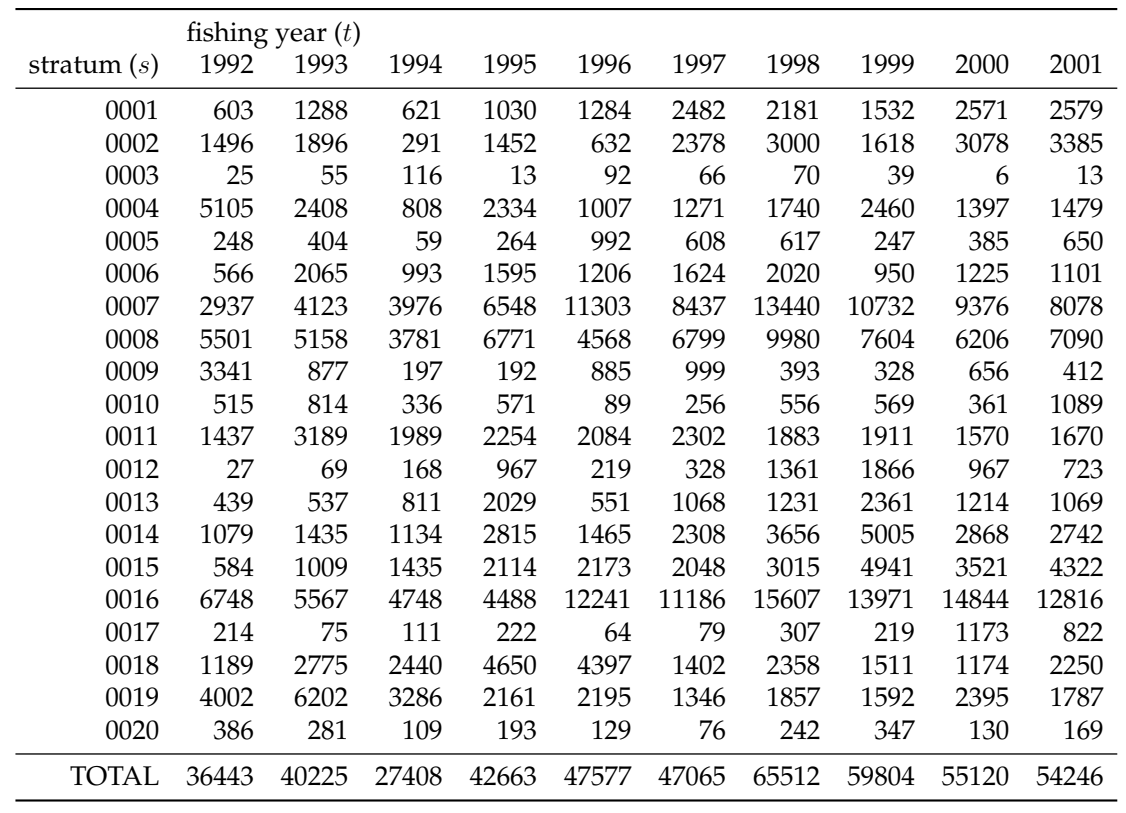

\begin{tabular}{rrrrrrrrrrrr}
\hline \multicolumn{1}{c}{ fishing year $(t)$} & & & & & & & & \\
stratum $(s)$ & 2002 & 2003 & 2004 & 2005 & 2006 & 2007 & 2008 & 2009 & 2010 & 2011 & 2012 \\
\hline 0001 & 2063 & 1823 & 1042 & 775 & 860 & 1335 & 980 & 773 & 1098 & 762 & \\
0002 & 2871 & 2882 & 3042 & 1687 & 778 & 1124 & 1047 & 905 & 789 & 1189 & 870 \\
0003 & 28 & 7 & 0 & 1315 & 1036 & 676 & 30 & 73 & 26 & 69 & 20 \\
0004 & 1282 & 1317 & 1392 & 732 & 571 & 660 & 937 & 372 & 844 & 651 & 1139 \\
0005 & 556 & 270 & 190 & 364 & 413 & 350 & 2114 & 305 & 591 & 92 & 642 \\
0006 & 1417 & 782 & 1177 & 361 & 365 & 680 & 398 & 560 & 809 & 430 & 367 \\
0007 & 7683 & 10237 & 9526 & 7094 & 5740 & 5438 & 5439 & 6964 & 5372 & 3763 & 3266 \\
0008 & 5069 & 6421 & 4873 & 5086 & 4084 & 3706 & 3475 & 2478 & 2137 & 4904 & 3730 \\
0009 & 500 & 1050 & 901 & 1503 & 2053 & 1928 & 2090 & 436 & 485 & 2147 & 2324 \\
0010 & 918 & 986 & 5698 & 1774 & 343 & 1968 & 2224 & 2592 & 304 & 82 & 72 \\
0011 & 3206 & 1226 & 1704 & 3661 & 745 & 1364 & 835 & 523 & 424 & 294 & 420 \\
0012 & 1151 & 1127 & 1319 & 1254 & 337 & 507 & 585 & 304 & 386 & 190 & 632 \\
0013 & 1826 & 1601 & 2106 & 2337 & 1997 & 1915 & 1042 & 533 & 861 & 773 & 1251 \\
0014 & 3326 & 3424 & 2823 & 2780 & 2230 & 1777 & 1649 & 1559 & 1567 & 1020 & 2659 \\
0015 & 3886 & 5113 & 2089 & 2476 & 2731 & 2794 & 1708 & 901 & 1620 & 685 & 1829 \\
0016 & 9462 & 12379 & 6727 & 4963 & 8155 & 7452 & 5183 & 6070 & 7862 & 6664 & 4396 \\
0017 & 145 & 191 & 74 & 332 & 142 & 401 & 415 & 144 & 694 & 334 & 153 \\
0018 & 1035 & 1182 & 264 & 299 & 807 & 1278 & 1309 & 260 & 212 & 179 & 312 \\
0019 & 1827 & 2590 & 1901 & 5099 & 8741 & 11196 & 11724 & 8288 & 9048 & 7582 & 10631 \\
0020 & 249 & 497 & 174 & 254 & 104 & 396 & 296 & 364 & 440 & 97 & 830 \\
\hline TOTAL & 47963 & 55344 & 47803 & 44415 & 42146 & 46470 & 43836 & 34610 & 35243 & 32243 & 36304 \\
\hline
\end{tabular}




\section{Appendix G: \\ Catch histories by fishing year (1992 - 2012) for four ratttail species, re- constructed for the Chatham Rise hoki fishery in survey core strata areas 0010 to 0020 . Listed with survey biomass indices provided in NIWA sum- marised survey data.}

\begin{tabular}{|c|c|c|c|c|}
\hline \multirow{3}{*}{$\begin{array}{l}\text { fishing } \\
\text { year }\end{array}$} & \multicolumn{4}{|c|}{ Javelinfish (Lepidorhynchus denticulatus) } \\
\hline & \multicolumn{3}{|c|}{ survey biomass index } & \multirow{2}{*}{$\begin{array}{r}\text { catch history } \\
\text { catch }(\mathrm{t})\end{array}$} \\
\hline & biomass (t) & $(95 \%$ CI $)$ & c.v. & \\
\hline 1992 & 8961.2 & $(6232.3,11690.2)$ & 0.15 & 1875.0 \\
\hline 1993 & 8716.0 & $(6849.4,10582.5)$ & 0.11 & 2020.0 \\
\hline 1994 & 16730.7 & $(12678.8,20782.6)$ & 0.12 & 2441.3 \\
\hline 1995 & 4726.1 & $(3604.7,5847.7)$ & 0.12 & 931.0 \\
\hline 1996 & 9616.1 & $(7533.1,11699.2)$ & 0.11 & 1746.8 \\
\hline 1997 & 5181.3 & $(4169.4,6193.3)$ & 0.10 & 1462.5 \\
\hline 1998 & 7854.6 & $(5870.1,9839.2)$ & 0.13 & 3510.6 \\
\hline 1999 & 10798.9 & $(8265.9,13331.8)$ & 0.12 & 4475.3 \\
\hline 2000 & 10965.6 & $(8054.3,13876.9)$ & 0.13 & 3553.7 \\
\hline 2001 & 15520.4 & $(12851.6,18189.3)$ & 0.09 & 5229.4 \\
\hline 2002 & 22758.7 & $(13827.9,31689.4)$ & 0.19 & 6428.9 \\
\hline 2003 & 13175.3 & $(10152.1,16198.5)$ & 0.12 & 5205.5 \\
\hline 2004 & 10953.7 & $(8743.5,13163.9)$ & 0.10 & 2843.0 \\
\hline 2005 & 11790.6 & $(8254.9,15326.3)$ & 0.15 & 2860.7 \\
\hline 2006 & 20379.6 & $(12371.5,28387.8)$ & 0.20 & 4565.9 \\
\hline 2007 & 14279.7 & $(10414,18145.4)$ & 0.13 & 3100.4 \\
\hline 2008 & 8381.4 & $(5105.1,11657.7)$ & 0.20 & 1594.4 \\
\hline 2009 & 20540.9 & $(16033.8,25047.9)$ & 0.11 & 3271.1 \\
\hline 2010 & 13924.8 & $(9964.3,17885.2)$ & 0.14 & 2205.2 \\
\hline 2011 & 7849.3 & $(5912.7,9786.0)$ & 0.12 & 1584.6 \\
\hline 2012 & 10547.1 & $(7065.6,14028.6)$ & 0.17 & 1897.4 \\
\hline
\end{tabular}

\begin{tabular}{rccr}
\hline \multicolumn{3}{c}{ Bollon's rattail (Coelorinchus bollonsi) } \\
\hline \multicolumn{3}{c}{ survey biomass index } & \multicolumn{2}{r}{ catch history } \\
biomass $(\mathrm{t})$ & $(95 \% \mathrm{CI})$ & c.v. & catch $(\mathrm{t})$ \\
\hline 11144.5 & $(8177.5,14111.6)$ & 0.13 & 3413.3 \\
11809.5 & $(9799.1,13820.0)$ & 0.85 & 2745.4 \\
16924.5 & $(13802.3,20046.8)$ & 0.92 & 3100.5 \\
5063.1 & $(3932.4,6193.8)$ & 0.11 & 1245.3 \\
8768.1 & $(6779.2,10756.9)$ & 0.11 & 2707.8 \\
6935.6 & $(4190.9,9680.2)$ & 0.20 & 1970.6 \\
9288.6 & $(7216.7,11360.8)$ & 0.11 & 4713.0 \\
13621.1 & $(10013.9,17228.3)$ & 0.13 & 6716.9 \\
12137.2 & $(10094.3,14180.2)$ & 0.84 & 5065.6 \\
14036.4 & $(11232.8,16840.0)$ & 0.10 & 6159.7 \\
16237.6 & $(12378.9,20096.2)$ & 0.12 & 6033.7 \\
8186.2 & $(6585.8,9786.6)$ & 0.98 & 3275.6 \\
7705.3 & $(6200.4,9210.2)$ & 0.98 & 2879.0 \\
5823.3 & $(4798.8,6847.7)$ & 0.88 & 1678.0 \\
10326.3 & $(8354.1,12298.6)$ & 0.96 & 3057.3 \\
8071.1 & $(6364.1,9777.9)$ & 0.11 & 2365.7 \\
7020.2 & $(5589.2,8451.2)$ & 0.11 & 1433.0 \\
12646.5 & $(10213.0,15079.9)$ & 0.96 & 2273.3 \\
10669.4 & $(8400.4,12938.4)$ & 0.11 & 2467.5 \\
3454.6 & $(2372.6,4536.7)$ & 0.16 & 832.8 \\
13513.6 & $(11212.1,15815.0)$ & 0.85 & 3209.6 \\
\hline
\end{tabular}

\begin{tabular}{lrcrr}
\hline \multicolumn{5}{c}{ Oliver's rattail (Coelorinchus oliverianus) } \\
\hline $\begin{array}{l}\text { fishing } \\
\text { year }\end{array}$ & \multicolumn{2}{c}{ survey biomass index } & \multicolumn{2}{c}{ catch history } \\
biomass $(\mathrm{t})$ & $(95 \% \mathrm{CI})$ & c.v. & catch $(\mathrm{t})$ \\
\hline 1992 & 539.2 & $(351.1,727.4)$ & 0.17 & 154.9 \\
1993 & 1152.1 & $(661.7,1642.5)$ & 0.21 & 280.2 \\
1994 & 2653.8 & $(1797.6,3509.9)$ & 0.16 & 568.9 \\
1995 & 592.5 & $(181.1,1003.9)$ & 0.35 & 150.5 \\
1996 & 1111.9 & $(489.8,1734.1)$ & 0.28 & 507.3 \\
1997 & 1158.0 & $(548.9,1767.2)$ & 0.26 & 497.9 \\
1998 & 1752.2 & $(734.1,2770.3)$ & 0.29 & 859.0 \\
1999 & 1168.3 & $(742.1,1594.5)$ & 0.18 & 608.2 \\
2000 & 1406.3 & $(790.3,2022.4)$ & 0.22 & 917.8 \\
2001 & 3373.1 & $(1776.4,4969.8)$ & 0.24 & 1302.6 \\
2002 & 1846.3 & $(1360.7,2331.8)$ & 0.13 & 961.5 \\
2003 & 1187.1 & $(374.2,1999.9)$ & 0.34 & 828.5 \\
2004 & 1937.8 & $(779.6,3096.1)$ & 0.30 & 673.5 \\
2005 & 1719.5 & $(695.8,2743.3)$ & 0.30 & 620.2 \\
2006 & 2055.8 & $(790.6,3321.0)$ & 0.31 & 528.7 \\
2007 & 2840.6 & $(845.5,4835.6)$ & 0.35 & 1243.7 \\
2008 & 1519.6 & $(973.3,2065.9)$ & 0.18 & 353.7 \\
2009 & 5139.9 & $(2810.8,7469.0)$ & 0.23 & 1468.0 \\
2010 & 1241.9 & $(563.9,1920.2)$ & 0.28 & 274.1 \\
2011 & 775.1 & $(484.1,1066.0)$ & 0.19 & 139.9 \\
2012 & 1411.2 & $(904.4,1918.0)$ & 0.18 & 354.7 \\
\hline
\end{tabular}

\begin{tabular}{rccr}
\hline \multicolumn{3}{r}{ Oblique banded rattail (Coelorinchus aspercephalus) } \\
\hline \multicolumn{3}{r}{ survey biomass index } & $\begin{array}{r}\text { catch history } \\
\text { catch }(\mathrm{t})\end{array}$ \\
\hline biomass $(\mathrm{t})$ & $(95 \% \mathrm{CI})$ & c.v. & 320.7 \\
1432.1 & $(650.0,2214.3)$ & 0.27 & 175.8 \\
1469.8 & $(0,3342.5)$ & 0.64 & 244.6 \\
1408.5 & $(991.9,1825.2)$ & 0.15 & 184.9 \\
527.2 & $(233.2,821.2)$ & 0.28 & 133.1 \\
798.9 & $(555.3,1042.4)$ & 0.15 & 176.7 \\
1477.3 & $(954.2,2000.5)$ & 0.18 & 226.7 \\
1441.9 & $(1039.9,1843.8)$ & 0.14 & 395.2 \\
1746.6 & $(1358.7,2134.5)$ & 0.11 & 697.0 \\
1672.2 & $(1287.3,2056.9)$ & 0.11 & 258.9 \\
1227.2 & $(810.8,1643.6)$ & 0.17 & 167.5 \\
978.2 & $(643.4,1313.1)$ & 0.17 & 154.8 \\
856.8 & $(668.1,1045.5)$ & 0.11 & 117.5 \\
841.0 & $(545.3,1136.8)$ & 0.18 & 297.9 \\
1185.3 & $(849.7,1520.9)$ & 0.14 & 397.3 \\
1489.4 & $(1101.8,1876.9)$ & 0.13 & 243.1 \\
978.0 & $(733.1,1222.9)$ & 0.13 & 257.0 \\
824.4 & $(553.9,1094.8)$ & 0.16 & 198.0 \\
1468.2 & $(1016.5,1919.9)$ & 0.15 & 123.4 \\
1447.2 & $(966.0,1928.7)$ & 0.17 & 463.8 \\
681.3 & $(377.6,985.1)$ & 0.22 & \\
1769.5 & $(1381.3,2157.7)$ & 0.11 &
\end{tabular}




\section{Appendix H: MLE Results, rattail species (CBO,CAS,COL)}

Table 1: Results of model fits for rattail species: Bollon's rattail (CBO)

\begin{tabular}{crrrrrrrrr}
\hline $\begin{array}{c}\text { procedure } \\
\text { type }\end{array}$ & optim & \multicolumn{1}{c}{ min. value } & \multicolumn{1}{c}{ parameter estimates: } & \multicolumn{3}{c}{ information criteria: } \\
\hline A & N-M & -9.19818 & 0.49 & 698697 & 0.01369 & 0.3914 & & & \\
& L-BFGS-U & -9.63595 & 0.10 & 153264 & 0.07220 & 0.3839 & & -19.135 & -17.724 \\
& SANN & -12.56769 & 2.78 & 4601673 & 0.00215 & 0.3921 & & & \\
\hline & MSB & -12.13324 & 2.71 & 2737722 & 0.00401 & 0.3532 & & & \\
\hline B & N-M & -8.87190 & 1.25 & 138867 & 0.01299 & 0.3975 & 604525 & & \\
& L-BFGS-U & failed to converge & & & & & & \\
& SANN & -9.56683 & -5.12 & 967730 & 0.01595 & 0.3913 & 628898 & & -8.833 \\
\hline
\end{tabular}

Table 2: MLE boostrap estimates: Bollon's (CBO), Proc. A (SANN)

\begin{tabular}{rrrrrr}
\hline & & \multicolumn{4}{c}{ bootstrap median and 95\% C. I. } \\
\cline { 3 - 6 } & ML estimate & median & lower & upper & median bias \\
\hline$r$ & 2.78 & 2.73 & -12.50 & 2.96 & 0.3 \\
$K$ & 4601673 & 4974534 & 2106241 & 10534013 & 0 \\
$q$ & 00.0022 & 0.0019 & 0.00009 & 0.0041 & 1.0 \\
\hline
\end{tabular}

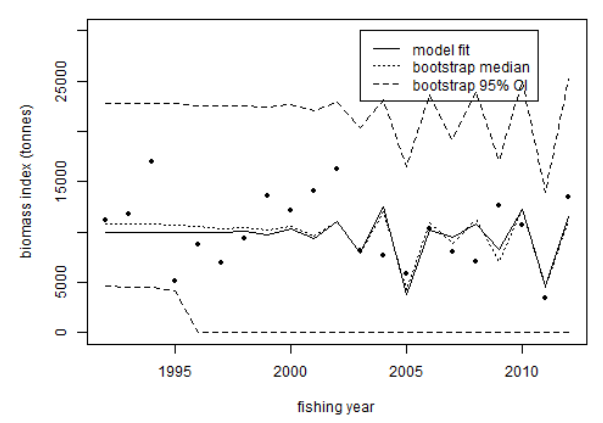

Figure 31: MLE: Bollon's rattail (CBO), proc. A, SANN. Plot of observed index $\left(I_{t}=\bullet\right)$ and model predicted index $\left(\hat{I}_{t}\right)$ with its boostrapped median and $95 \%$ C.I.

(a)

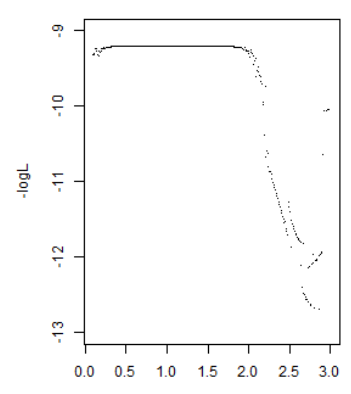

Figure 32: Plot of univariate likelihood profiles for $r$ : Bollon's attail (CBO), proc. A, (SANN) 
Table 3: Results of model fits for rattail species: Oblique banded rattail (CAS)

\begin{tabular}{|c|c|c|c|c|c|c|c|c|c|}
\hline \multirow{2}{*}{$\begin{array}{c}\text { procedure } \\
\text { type }\end{array}$} & \multirow{2}{*}{$\begin{array}{l}\text { optim } \\
\text { method }\end{array}$} & \multirow{2}{*}{$\begin{array}{c}\text { min. value } \\
-\log L\end{array}$} & \multicolumn{5}{|c|}{ parameter estimates: } & \multicolumn{2}{|c|}{ information criteria: } \\
\hline & & & $r$ & $K$ & $q$ & $\sigma_{u}$ & $B_{1}$ & AIC & AICc \\
\hline \multirow[t]{4}{*}{$\mathrm{A}$} & N-M & -12.72841 & 0.49 & 72561 & 0.01615 & 0.3308 & & & \\
\hline & L-BFGS-U & -12.90283 & 0.16 & 23815 & 0.05140 & 0.3290 & & & \\
\hline & SANN & -14.05902 & 2.46 & 965056 & 0.00122 & 0.3125 & & -22.118 & -20.706 \\
\hline & MSB & -14.01864 & 2.45 & 679781 & 0.00172 & 0.3575 & & & \\
\hline \multirow[t]{4}{*}{ B } & N-M & -12.03683 & 0.50 & 153341 & 0.00777 & 0.3301 & 125461 & & \\
\hline & L-BFGS-U & failed to cor & iverge & & & & & & \\
\hline & SANN & -12.76471 & -4.18 & 2420885 & 0.02000 & 0.3325 & 60777 & -17.529 & -15.029 \\
\hline & MSB & -12.76152 & -3.78 & 90734 & 0.01600 & 0.3350 & 74613 & & \\
\hline
\end{tabular}

Table 4: MLE boostrap estimates: Oblique banded rattail (CAS), proc. A (SANN)

\begin{tabular}{rrrrrr}
\hline & & \multicolumn{3}{c}{ bootstrap median and 95\% C. I. } \\
\cline { 3 - 6 } & ML estimate & median & lower & upper & median bias \\
\hline$r$ & 2.46 & 2.37 & -11.67 & 2.69 & 0.26 \\
$K$ & 965056 & 656038 & 317089 & 1384757 & 0 \\
$q$ & 0.00122 & 0.00179 & 0.00083 & 0.00367 & 1.00 \\
\hline
\end{tabular}

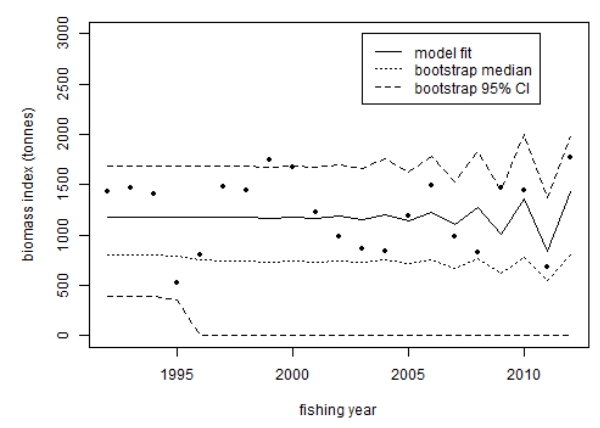

Figure 33: MLE: Oblique banded rattail (CAS), proc. A, SANN. Plot of observed index $\left(I_{t}=\bullet\right)$ and model predicted index $\left(\hat{I}_{t}\right)$ with its boostrapped median and 95\% C.I.

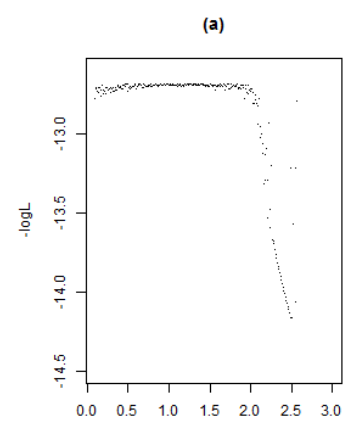

Figure 34: Plot of univariate likelihood profiles for $r$ : Oblique banded rattail (CAS), proc. A, SANN. 
Table 5: Results of model fits for rattail species: Oliver's rattail (COL)

\begin{tabular}{crrrrrrrrr}
\hline $\begin{array}{c}\text { procedure } \\
\text { type }\end{array}$ & optim & \multicolumn{2}{c}{ min. value } & \multicolumn{3}{c}{ parameter estimates: } & \multicolumn{3}{c}{ information criteria: } \\
\hline method & \multicolumn{1}{c}{$-\log \mathrm{L}$} & \multicolumn{1}{c}{$r$} & \multicolumn{1}{c}{$K$} & $q$ & $\sigma_{u}$ & $B_{1}$ & AIC & AICc \\
\hline & N-M & -2.83373 & 0.50 & 521763 & 0.00289 & & & \\
& L-BFGS-U & -2.84782 & 1.74 & 1308025 & 0.00153 & & & \\
& SANN & -2.82033 & -7.91 & 1872653 & 0.00081 & & & \\
& MSB & -2.84816 & 1.76 & 2964480 & 0.00051 & & 0.304 & 1.715 \\
\hline B & N-M & -3.69183 & 0.25 & 161408 & 0.00986 & 132116 & & \\
& L-BFGS-U & -5.35380 & 0.30 & 115989 & 0.01580 & 48721 & -2.708 & -0.208 \\
& SANN & -4.16483 & 0.04 & 1539190 & 0.00095 & 120727 & & \\
& MSB & -5.33870 & 0.28 & 449472 & 0.00409 & 192062 & & \\
\hline
\end{tabular}

Table 6: MLE boostrap estimates: Oliver's rattail (COL), proc. B (L-BFSG-U)

\begin{tabular}{crrrrr}
\hline & & \multicolumn{5}{c}{ bootstrap median and 95\% C. I. } \\
\cline { 3 - 6 } & ML estimate & median & lower & upper & median bias \\
\hline$r$ & 0.30 & 0.19 & 0.02 & 1.09 & 0.68 \\
$K$ & 115989 & 1166354 & 125553 & 29290993 & 0.015 \\
$q$ & 0.01585 & 0.00439 & 0.00108 & 0.21949 & 0.73 \\
$B_{1}$ & 48721 & 159002 & 5481 & 717119 & 0.30 \\
\hline
\end{tabular}

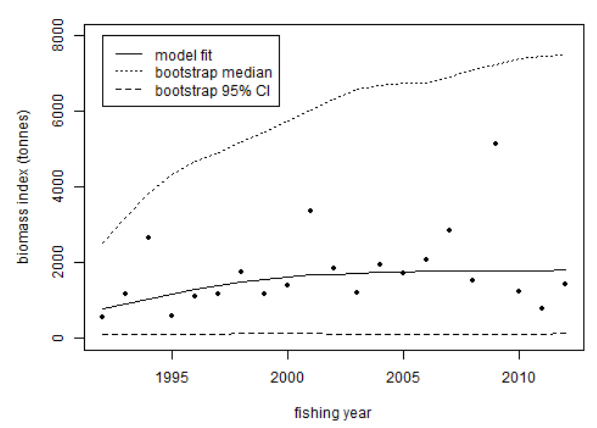

Figure 35: MLE: Oliver's rattail (COL), proc. B (L-BFSG-U). Plot of observed index $\left(I_{t}=\bullet\right)$ and model predicted index $\left(\hat{I}_{t}\right)$ with its boostrapped median and $95 \%$ C.I.

(a)

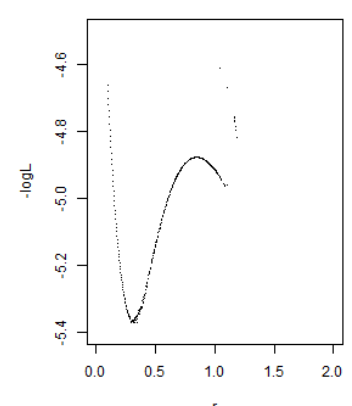

(b)

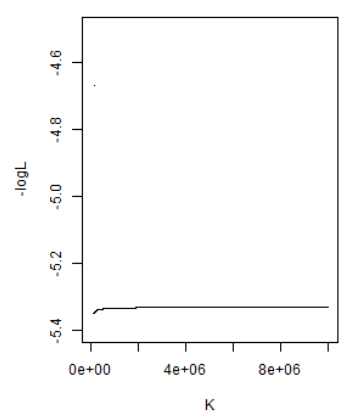

Figure 36: MLE plot of univariate likelihood profiles for $r$ and $K$ : Oliver's rattail (COL), proc. B (L-BFSG-U). 


\section{Appendix I: WinBUGS code for state-space production model}

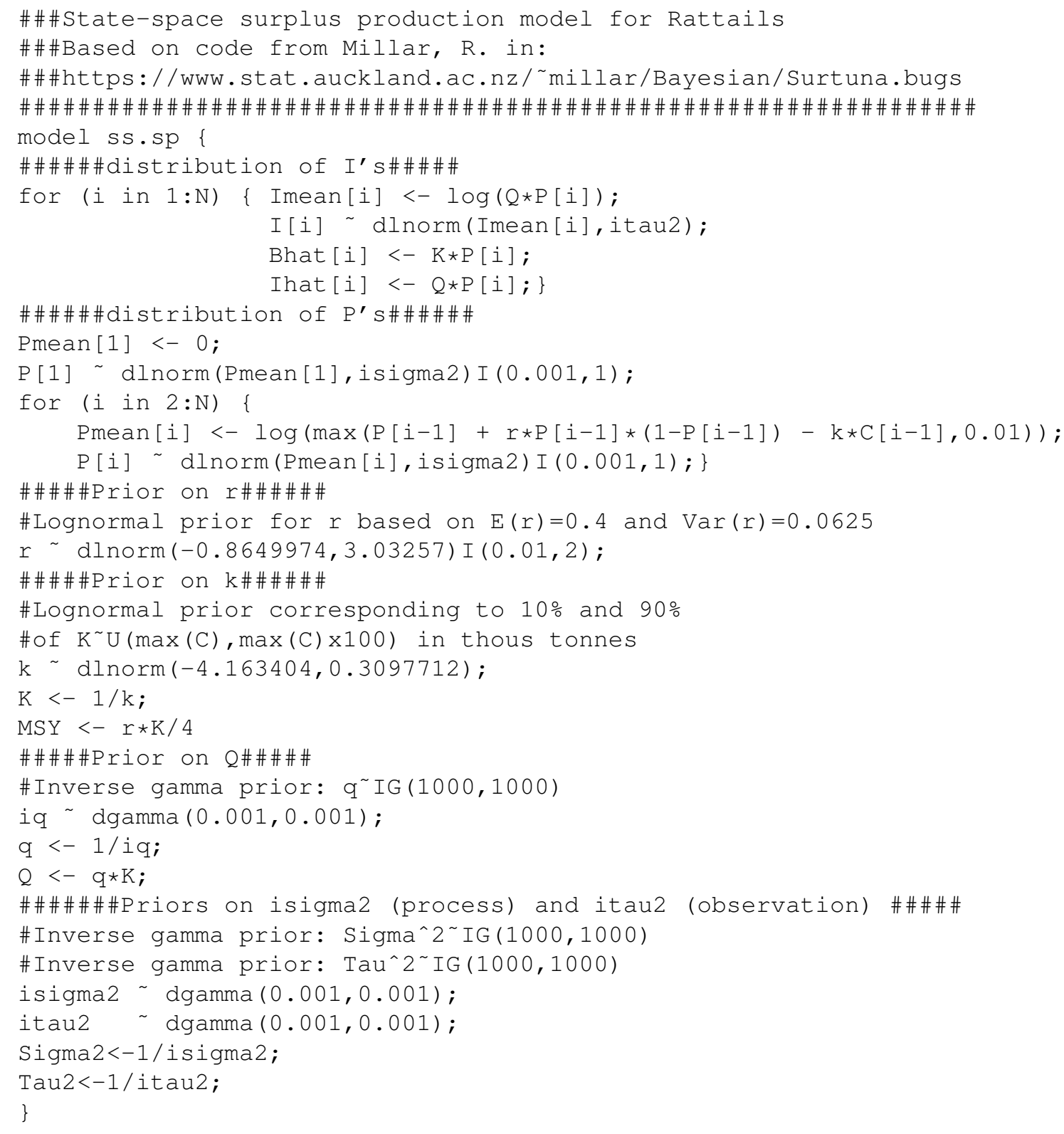




\section{Appendix J: Convergence diagnostic plots for $\mathrm{CBO}, \mathrm{COL}$ and CAS}
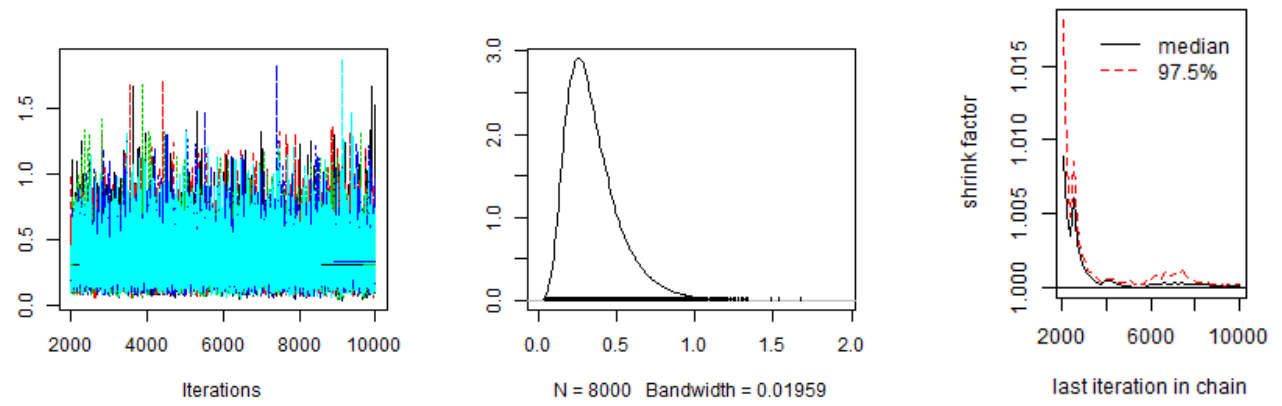

(a) Trace plot, density plot and Gelman-Rubin diagnostic plot.

(b) label 2

Figure 37: Bollon's rattail (CBO): Convergence checks of posterior sample of $r$.
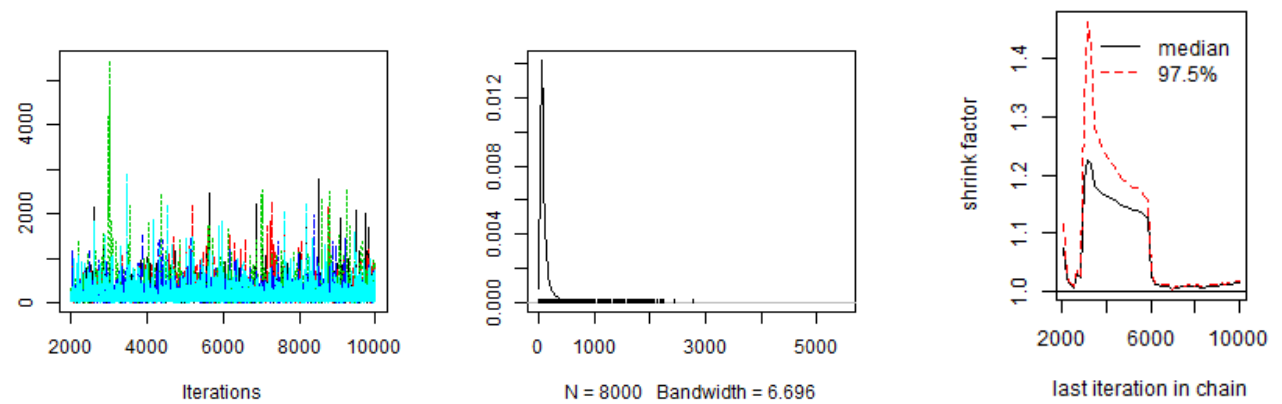

(a) Trace plot, density plot and Gelman-Rubin diagnostic plot.

Figure 38: Bollon's rattail (CBO): Convergence checks of posterior sample of $K$.
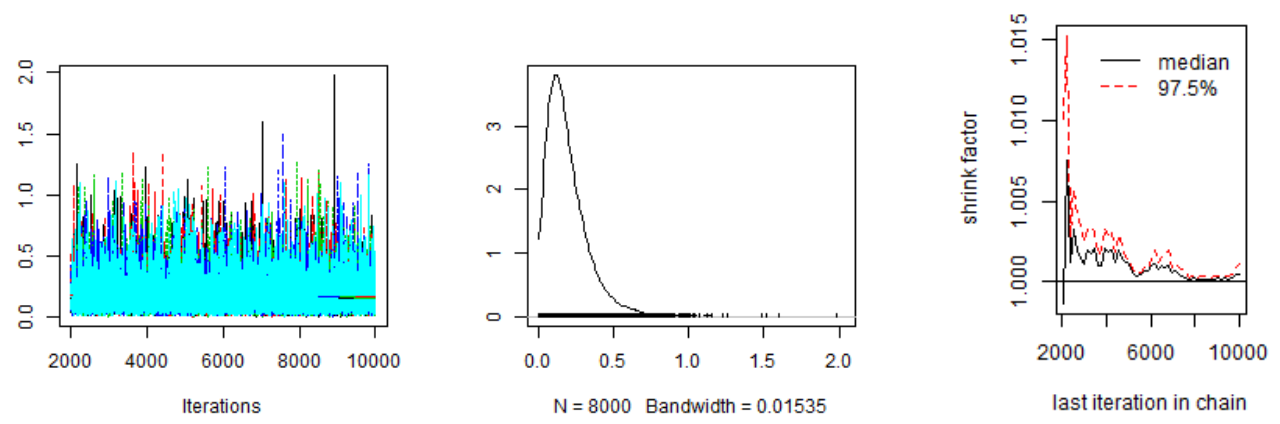

(a) Trace plot, density plot and Gelman-Rubin diagnostic plot.

Figure 39: Bollon's rattail (CBO): Convergence checks of posterior sample of $q$. 

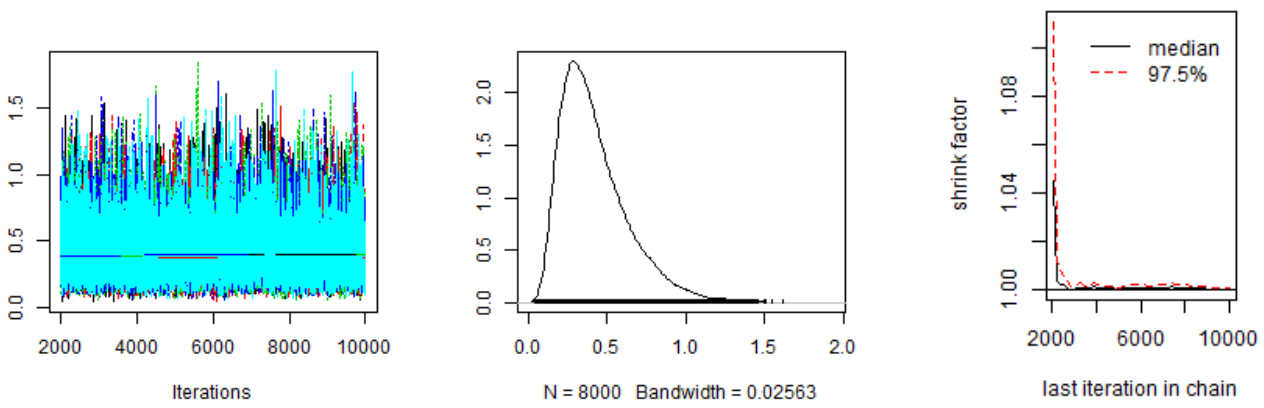

(a) Trace plot, density plot and Gelman-Rubin diagnostic plot.

Figure 40: Oliver's rattail (COL): Convergence checks of posterior sample of $r$.
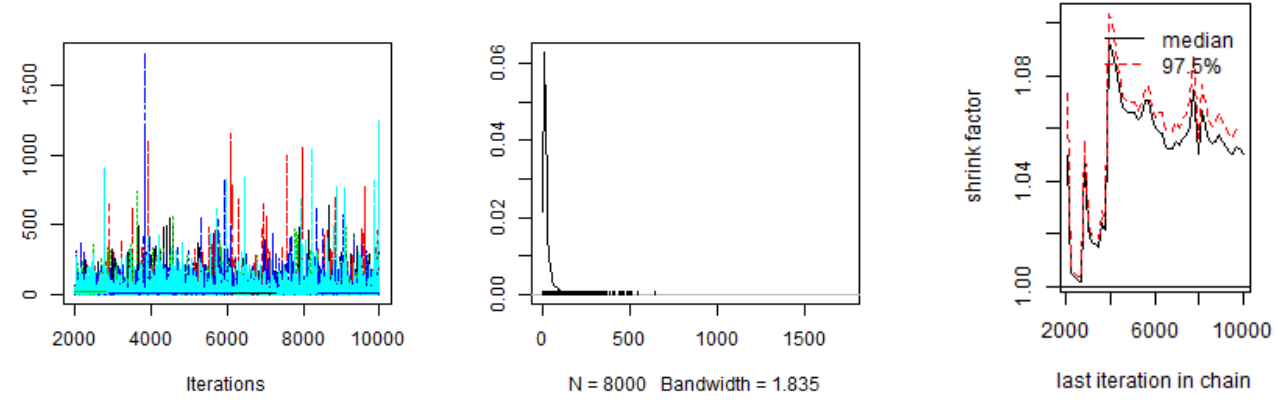

(a) Trace plot, density plot and Gelman-Rubin diagnostic plot.

Figure 41: Oliver's rattail (COL): Convergence checks of posterior sample of $K$.
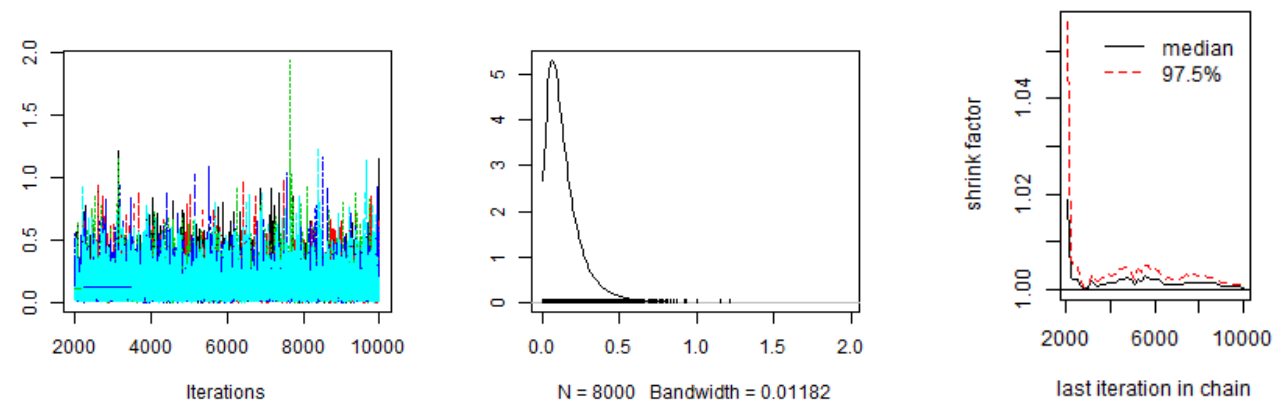

(a) Trace plot, density plot and Gelman-Rubin diagnostic plot.

Figure 42: Oliver's rattail (COL): Convergence checks of posterior sample of $q$. 

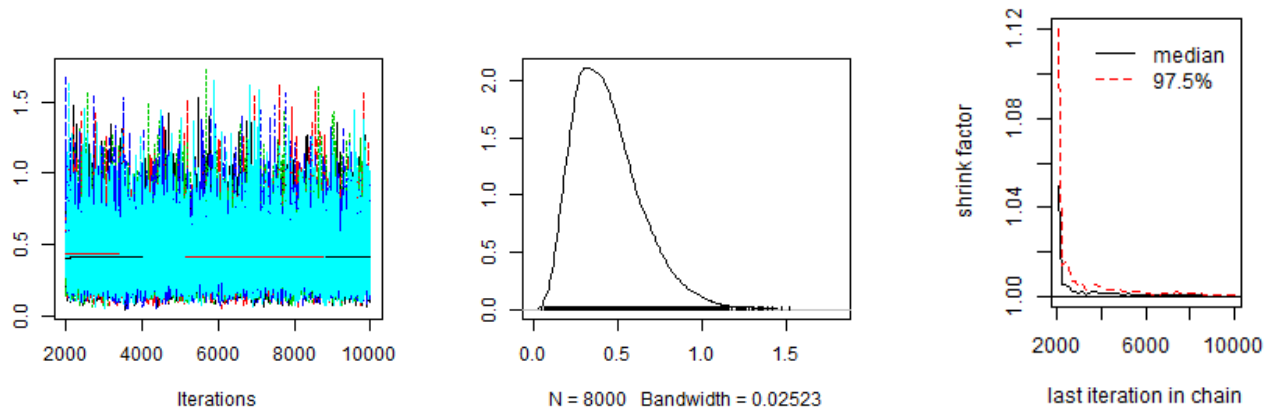

(a) Trace plot, density plot and Gelman-Rubin diagnostic plot.

Figure 43: Oblique banded rattail (CAS): Convergence checks of posterior sample of $r$.
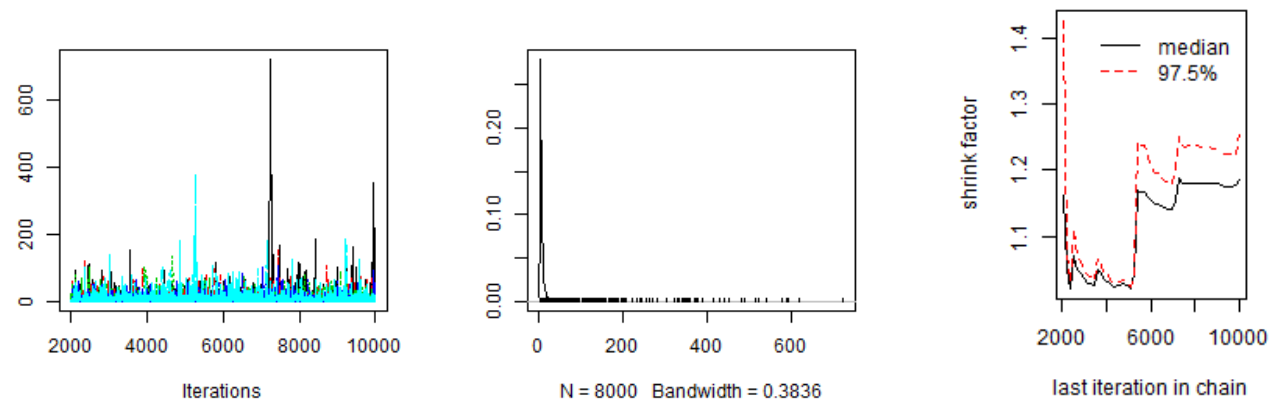

(a) Trace plot, density plot and Gelman-Rubin diagnostic plot.

Figure 44: Oblique banded rattail (CAS): Convergence checks of posterior sample of $K$.
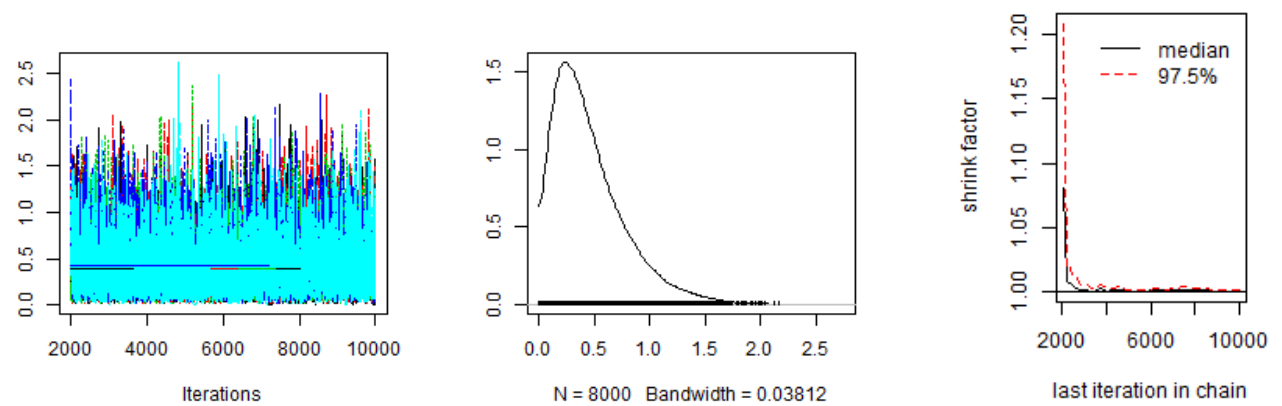

(a) Trace plot, density plot and Gelman-Rubin diagnostic plot.

Figure 45: Oblique banded rattail (CAS): Convergence checks of posterior sample of $q$. 


\section{Appendix K: Posterior estimates for CBO, COL and CAS}

Table 7: Bollon's rattail (CBO): Posterior estimates

\begin{tabular}{|c|c|c|c|c|c|c|c|c|c|}
\hline & \multicolumn{3}{|c|}{$r$ prior: $\mathrm{E}(r)=0.1$} & \multicolumn{3}{|c|}{$r$ prior: $\mathrm{E}(r)=0.4$} & \multicolumn{3}{|c|}{$r$ prior: $\mathrm{E}(r)=0.8$} \\
\hline & mean & median & $\begin{array}{c}95 \% \\
\text { HPD interval }\end{array}$ & mean & median & $\begin{array}{c}95 \% \\
\text { HPD interval }\end{array}$ & mean & median & $\begin{array}{c}95 \% \\
\text { HPD interval }\end{array}$ \\
\hline \multicolumn{10}{|c|}{$K$ prior: upper limit $\max (C) \times 20$} \\
\hline$r$ & 0.12 & 0.07 & $(0.01,0$ & 0.33 & 0.3 & $(0.08,0.66)$ & 0.68 & 0.67 & $(0.34,1.1)$ \\
\hline$K$ & 146 & 116 & (19? & 80 & 65 & $(17$ & 47 & 40 & $00)$ \\
\hline$q$ & 0.1387 & 0.113 & .0102 & 2291 & 0.1955 & $(0.0187,0$. & 0.3527 & 0.2956 & $(0.0479,0.8116)$ \\
\hline$\sigma_{u}^{2}$ & 0.1664 & 0.1553 & $(0.0565,0.3162)$ & 0.1658 & 0.0554 & $(0.0403,0.3233)$ & 0.1652 & 0.1586 & $(0.0003,0.3044)$ \\
\hline$\sigma_{v}^{2}$ & 0.0183 & 0.0047 & $(0.0001,0.0765)$ & 0.0225 & 0.0049 & $(0.0002,0.1068)$ & 0.0343 & 0.0039 & $(0.0001,0.2096)$ \\
\hline MSY & 3.1 & 2.3 & $(0.1,8.1)$ & 5.8 & 4.8 & $(1.5,12.6)$ & 7.7 & 6.6 & $(2.6,15.6)$ \\
\hline \multicolumn{10}{|c|}{$K$ prior: upper limit $\max (C) \times 100$} \\
\hline$r$ & 0.09 & 0.05 & $(0.01,0$ & 0.3 & 0.27 & $\left(0.0^{\prime}\right.$ & 0.67 & 0.64 & $(0.3$ \\
\hline$K$ & 338 & 178 & $(161$ & 154 & 83 & $(17$ & 63 & 45 & $(13$ \\
\hline$q$ & 0.0917 & 0712 & .0018 & 1783 & 0.147 & $(0.0033,0.435)$ & 0.3125 & 0.2606 & $(0.0152,0.7461)$ \\
\hline $\operatorname{sigma}_{u}^{2}$ & 0.1654 & 0.1544 & $(0.0560,0.3036)$ & 0.1642 & 0.1549 & $(0.0451,0.3276)$ & 0.1659 & 0.159 & $(0.0003,0.3037)$ \\
\hline$\sigma_{v}^{\alpha}$ & 0.0172 & 0.0043 & $(0.0001,0.0727)$ & 0.0229 & 0.0045 & $(0.0001,0.1094)$ & 0.0343 & 0.0035 & $(0.0001,0.2134)$ \\
\hline MSY & 5.1 & 2.8 & $(0.1,15.9)$ & 9.5 & 5.6 & $(1.2,2$ & 9.8 & 7.2 & $(2.4,21.4)$ \\
\hline \multicolumn{10}{|c|}{$K$ prior: upper limit $\max (C) \times 1000$} \\
\hline$r$ & 0.07 & 0.04 & $(0.01,0.2$ & 0.29 & 0.25 & $(0.07$, & 0.66 & 0.63 & $(0.33$ \\
\hline$K$ & 1177 & 288 & $(20,5018)$ & 285 & 99 & $(12,1187)$ & 87 & 47 & $(11,191)$ \\
\hline$q$ & 0.0649 & 0.0435 & $(0.0004,0.1975)$ & 0.1533 & 0.1219 & $(0.0018,0.4002)$ & 0.2979 & 0.2469 & $(0.0041,0.7155)$ \\
\hline$\sigma_{u}^{2}$ & 0.1636 & 0.1525 & $(0.0616,0.3065)$ & 0.1627 & 0.1541 & $(0.0003,0.2841)$ & 0.1637 & 0.1579 & $(0.0003,0.3015)$ \\
\hline$\sigma_{v}^{2}$ & 0.01823 & 0.0047 & $(0.0001,0.0757)$ & 0.0258 & 0.0048 & $(0.0001,0.1304)$ & 0.0374 & 0.0034 & $(0.0001,0.2383)$ \\
\hline MSY & 15.1 & 3.8 & $(0.2,57.3)$ & 16.2 & 6.4 & $(0.7,60.2)$ & 13.3 & 7.5 & $(2.2,27.6)$ \\
\hline
\end{tabular}



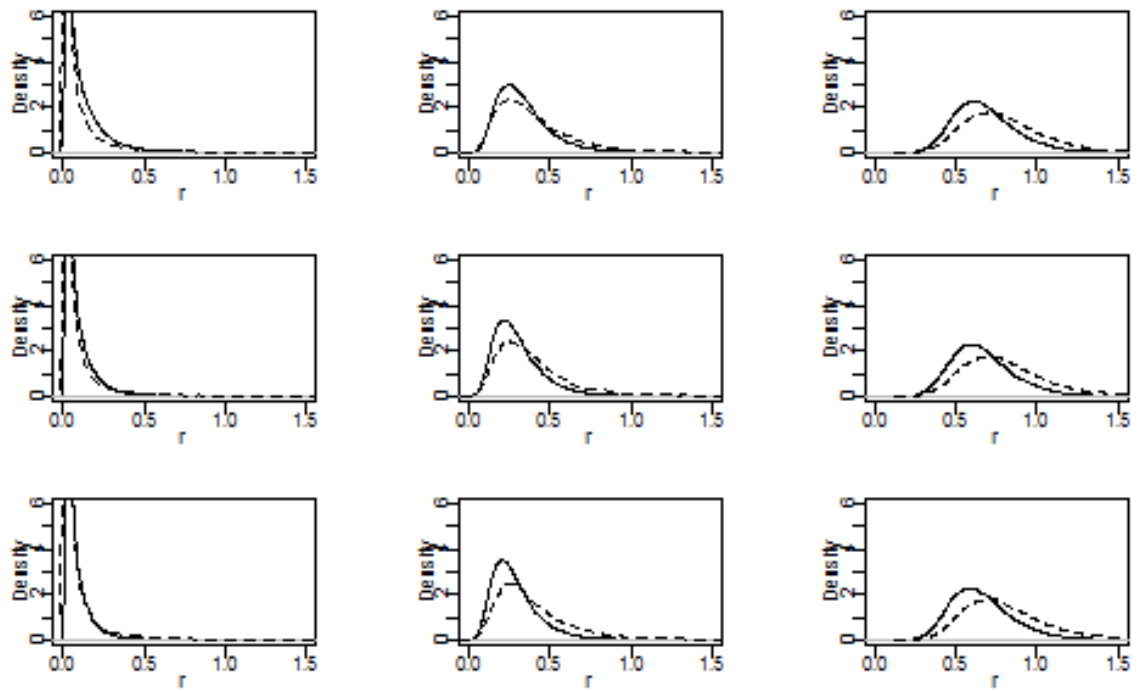

Figure 46: Bollon's rattail (CBO): prior and posterior distributions for $r$. Solid line is posterior distribution, dashed line is prior distribution. Left to right: increasing value of $\mathrm{E}(r)=(0.1,0.4,0.8)$ specified in $r$ prior. Top to bottom: increasing upper-limit set in $K$ prior $(\max (C) \times 20, \max (C) \times 100, \max (C) \times 1000)$.
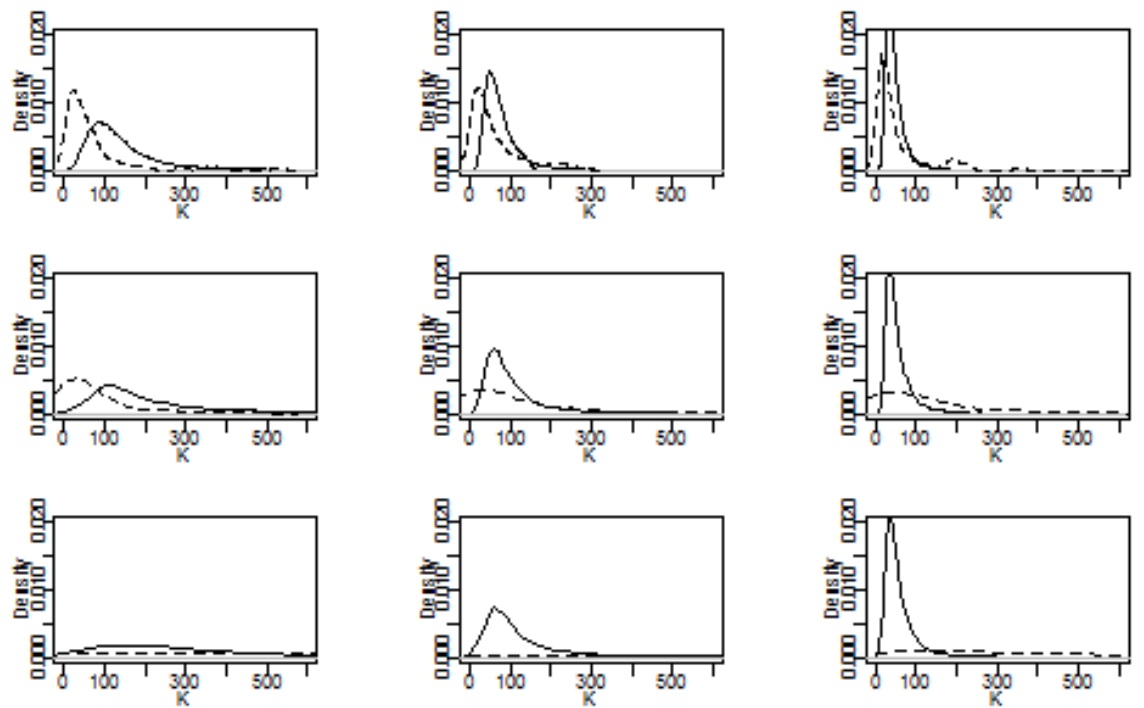

Figure 47: Bollon's rattail (CBO): prior and posterior distributions for $K$. Solid line is posterior distribution, dashed line is prior distribution. Left to right: increasing value of $\mathrm{E}(r)=(0.1,0.4,0.8)$ specified in $r$ prior. Top to bottom: increasing upper-limit set in $K$ prior $(\max (C) \times 20, \max (C) \times 100, \max (C) \times 1000)$. 


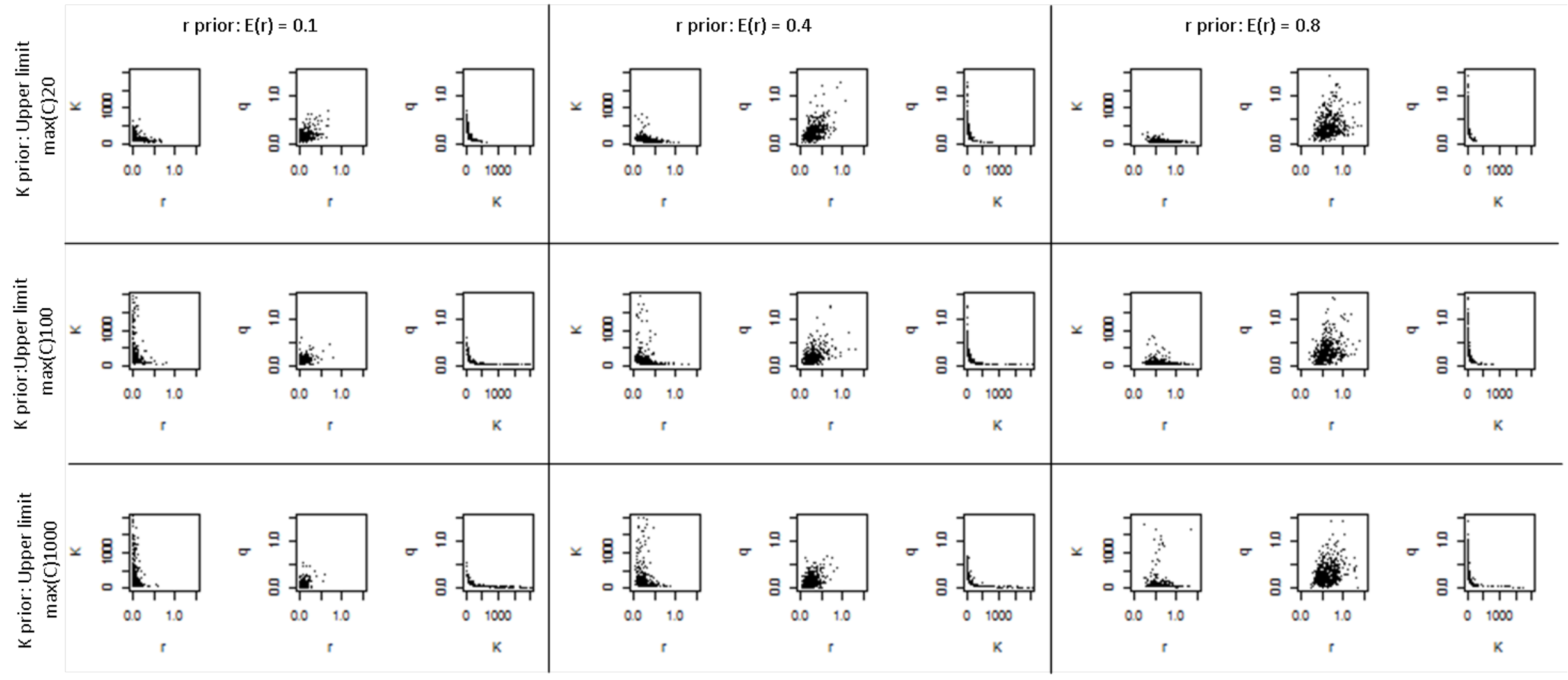

Figure 48: CBO: parameter correlations r,K,q 

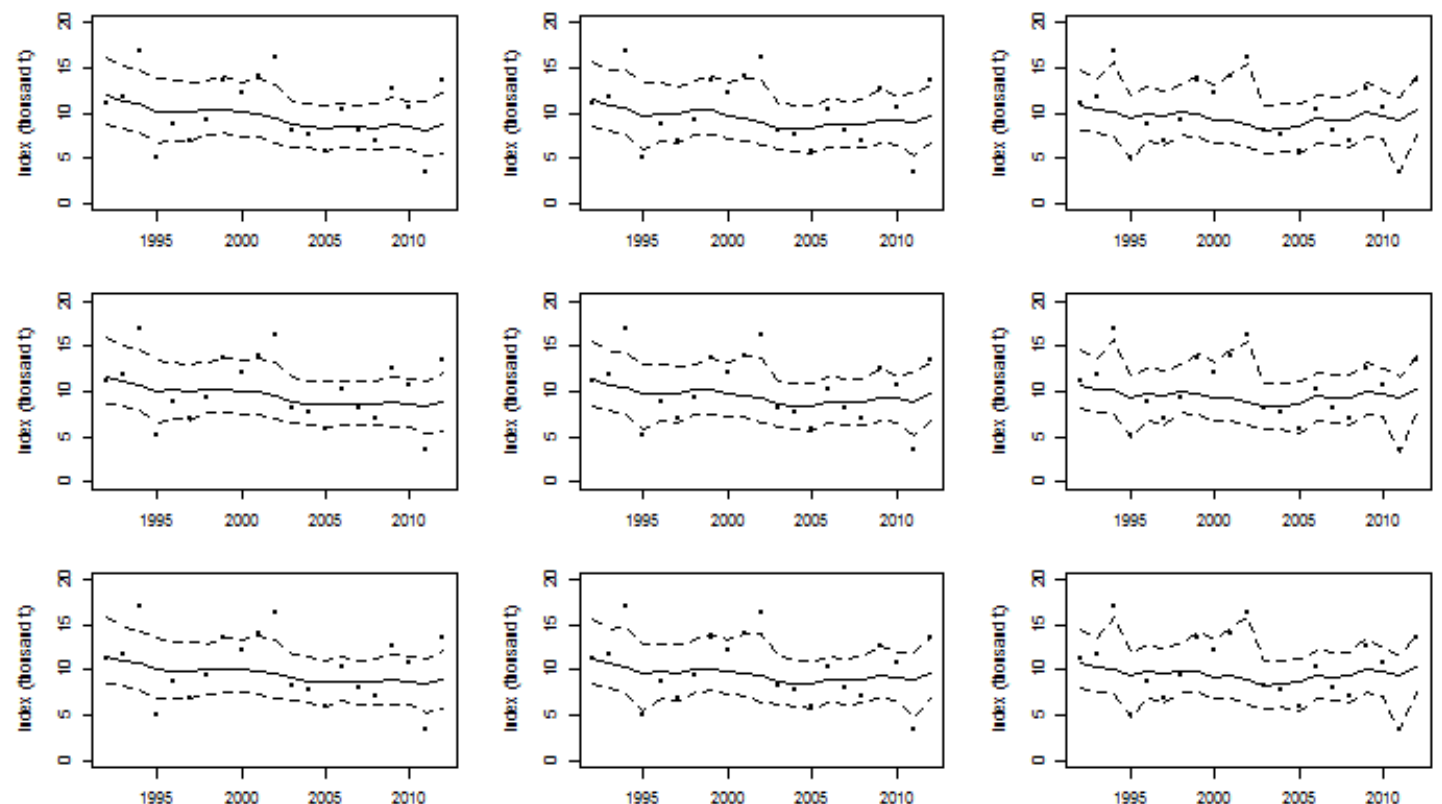

Figure 49: Bollon's rattail (CBO): Plot of observed index $\left(I_{t}=\bullet\right)$ and posterior median for the model predicted index (solid line) with its 95\%HPD interval (dashed line).Left to right: increasing value of $\mathrm{E}(r)=(0.1,0.4,0.8)$ specified in $r$ prior. Top to bottom: increasing upper-limit set in $K$ prior $(\max (C) \times 20, \max (C) \times 100, \max (C) \times 1000)$.
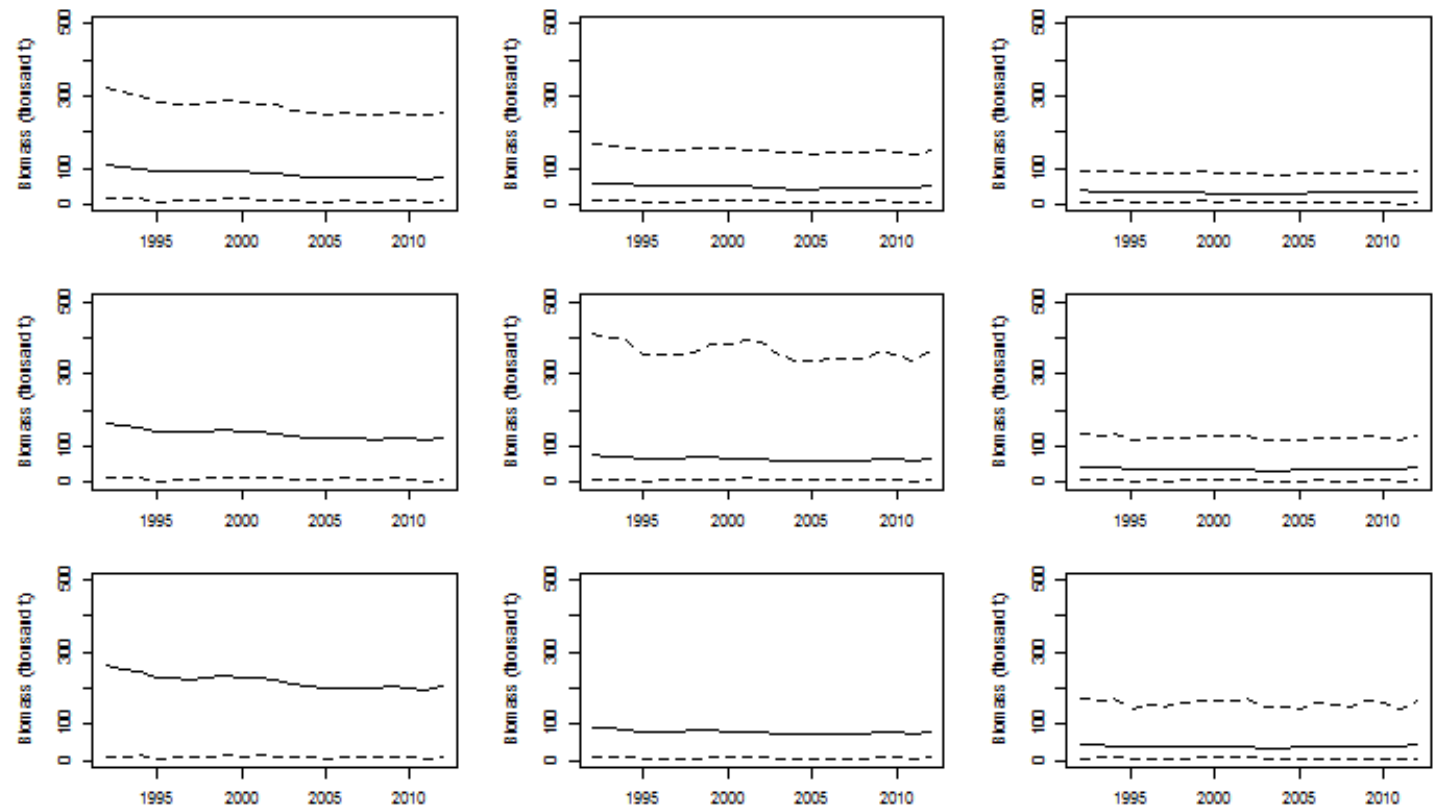

Figure 50: Bollon's rattail (CBO): Plot of the posterior median for the model predicted index (solid line) with its 95\%HPD interval (dashed line).Left to right: increasing value of $\mathrm{E}(r)=(0.1,0.4,0.8)$ specified in $r$ prior. Top to bottom: increasing upper-limit set in $K$ prior $(\max (C) \times 20, \max (C) \times 100, \max (C) \times 1000)$. 
106

Table 8: Oliver's rattail (COL): Posterior estimates

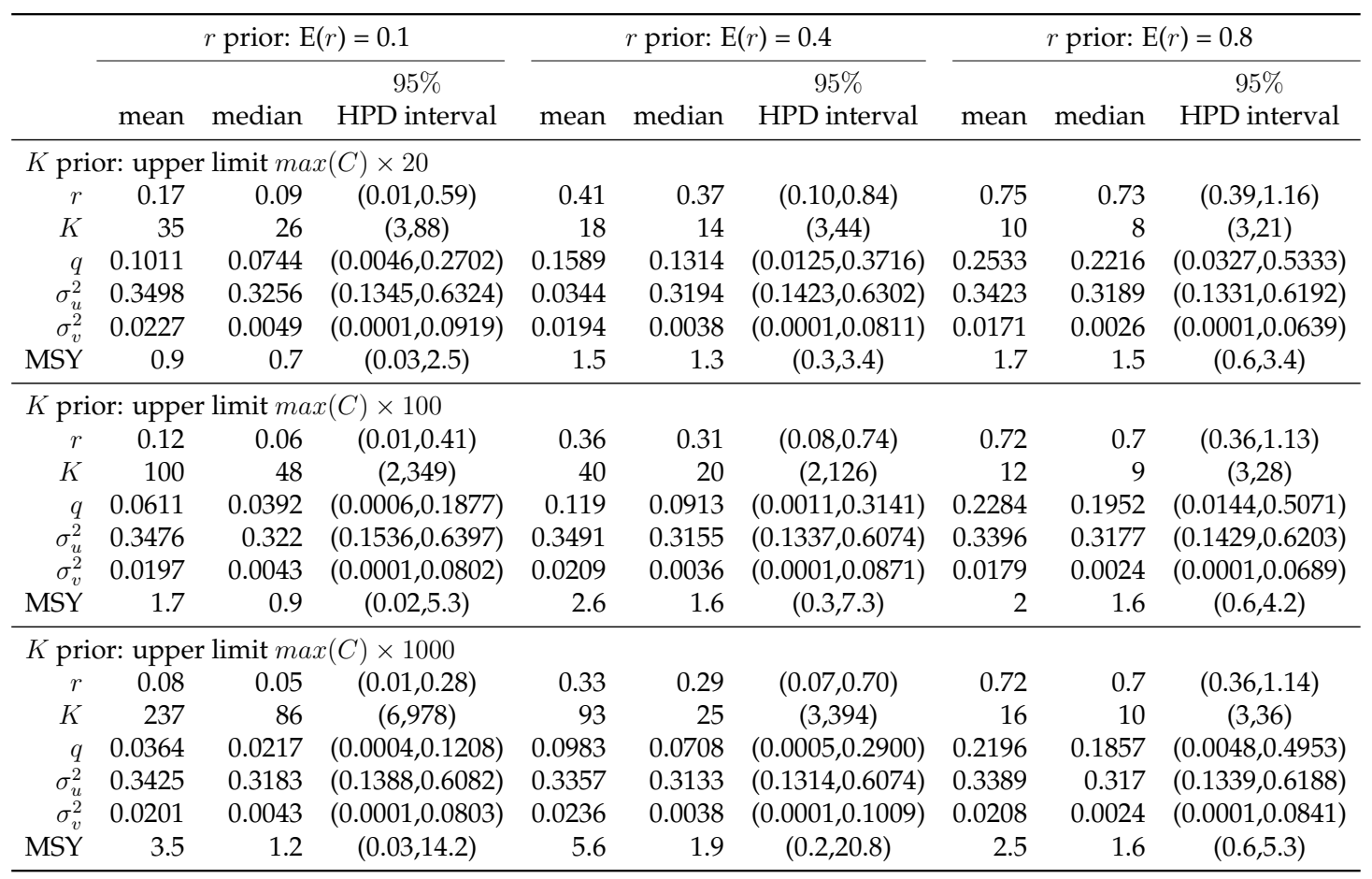



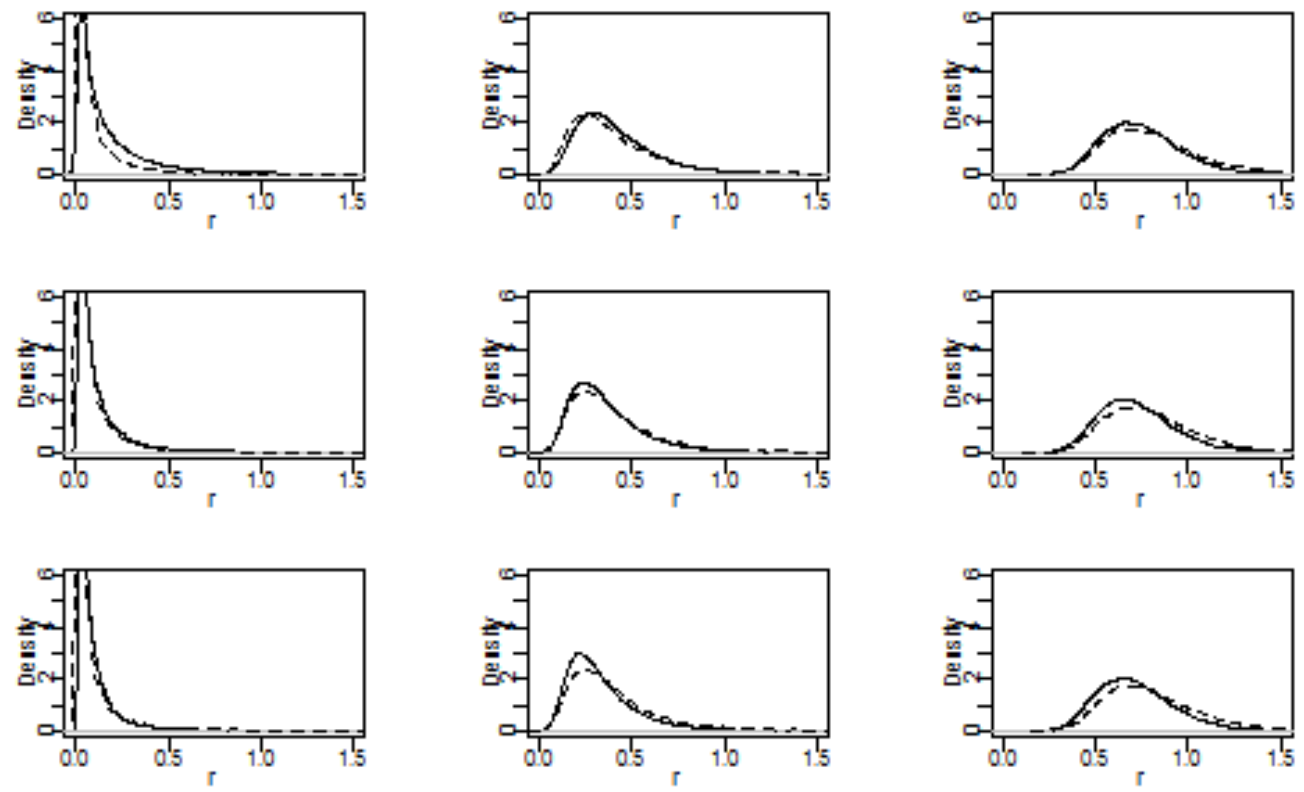

Figure 51: Oliver's rattail (COL): prior and posterior distributions for $r$. Solid line is posterior distribution, dashed line is prior distribution. Left to right: increasing value of $\mathrm{E}(r)=(0.1,0.4,0.8)$ specified in $r$ prior. Top to bottom: increasing upper-limit set in $K$ prior $(\max (C) \times 20, \max (C) \times 100, \max (C) \times 1000)$.
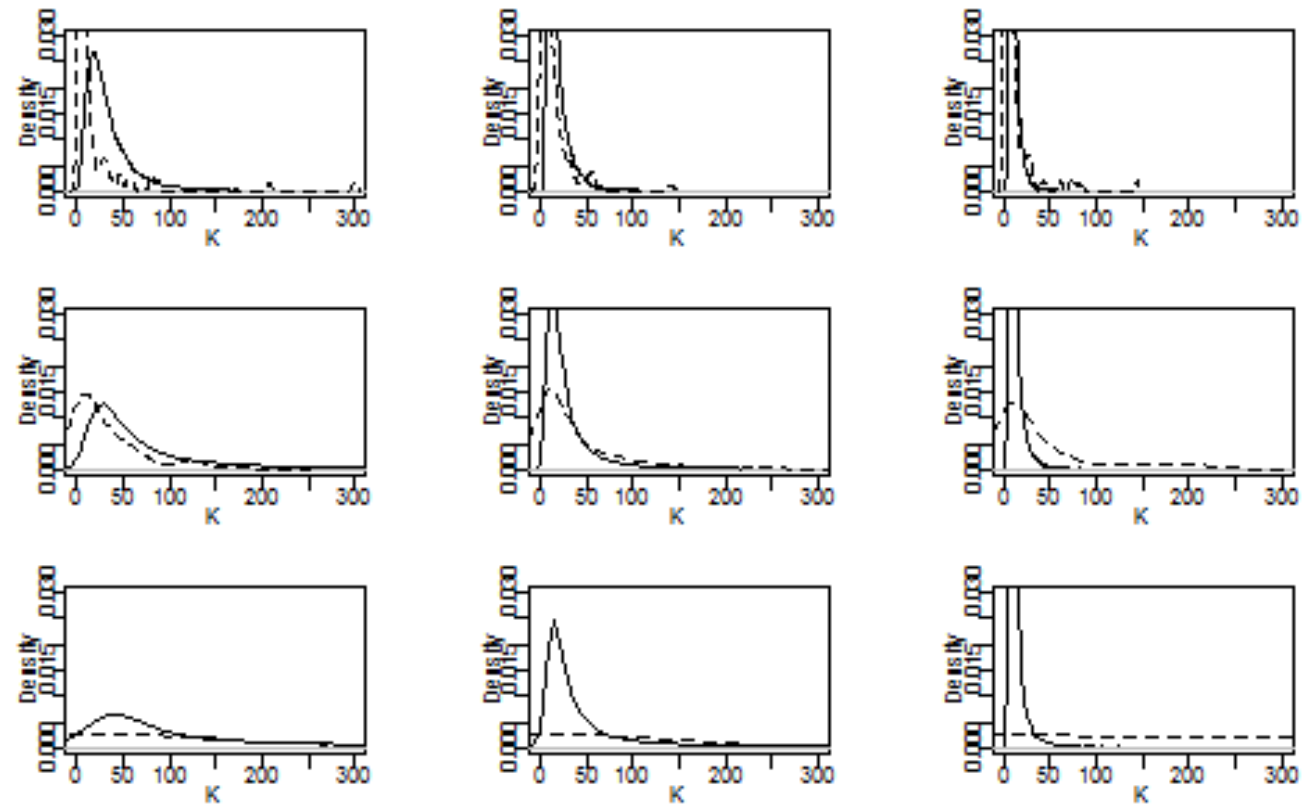

Figure 52: Oliver's rattail (COL): prior and posterior distributions for $K$. Solid line is posterior distribution, dashed line is prior distribution. Left to right: increasing value of $\mathrm{E}(r)=(0.1,0.4,0.8)$ specified in $r$ prior. Top to bottom: increasing upper-limit set in $K$ prior $(\max (C) \times 20, \max (C) \times 100, \max (C) \times 1000)$. 


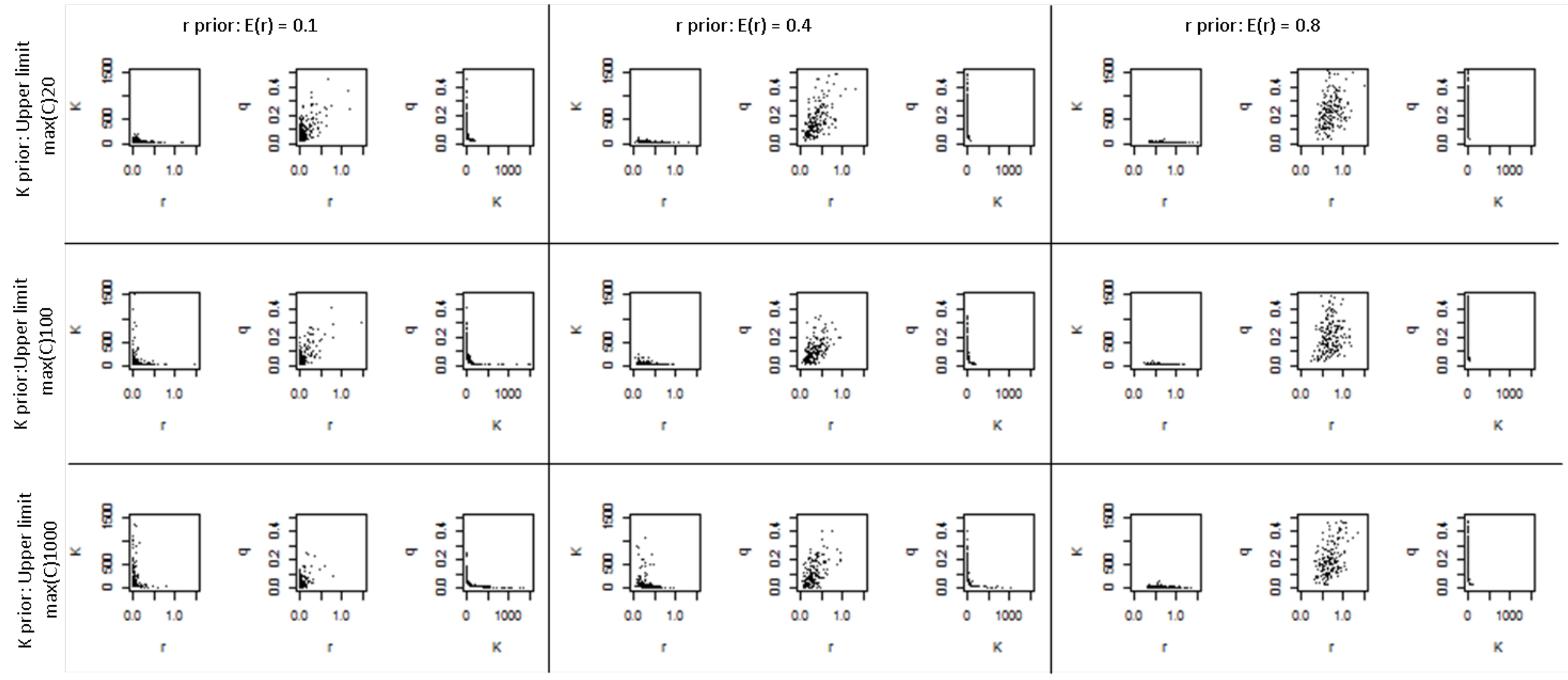

Figure 53: COL: parameter correlations $r, \mathrm{~K}, \mathrm{q}$ 

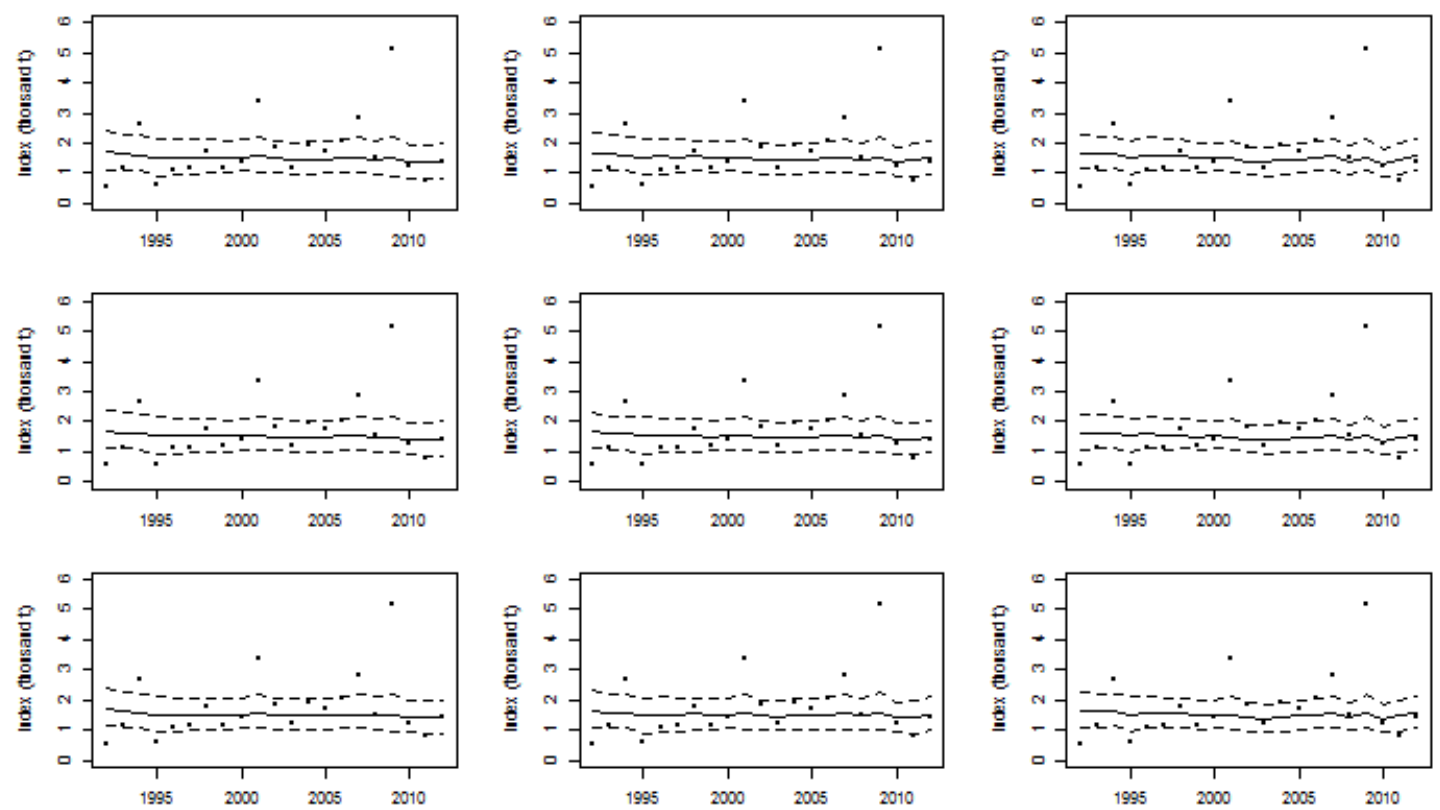

Figure 54: Oliver's rattail (COL): Plot of observed index $\left(I_{t}=\bullet\right)$ and posterior median for the model predicted index (solid line) with its 95\%HPD interval (dashed line).Left to right: increasing value of $\mathrm{E}(r)=(0.1,0.4,0.8)$ specified in $r$ prior. Top to bottom: increasing upper-limit set in $K$ prior $(\max (C) \times 20, \max (C) \times 100, \max (C) \times 1000)$. 

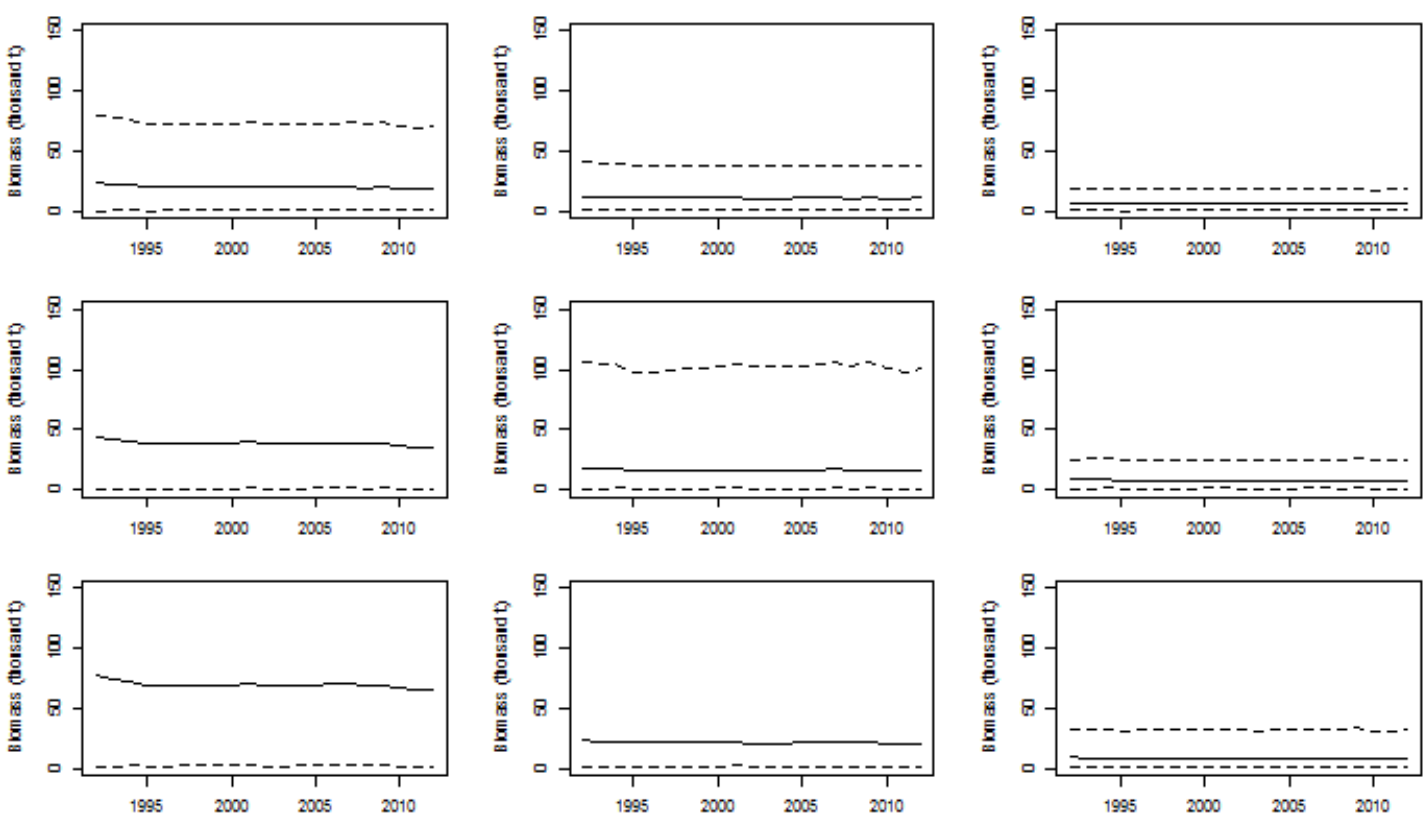

Figure 55: Oliver's rattail (COL): Plot of the posterior median for the model predicted index (solid line) with its $95 \%$ HPD interval (dashed line).Left to right: increasing value of $\mathrm{E}(r)=(0.1,0.4,0.8)$ specified in $r$ prior. Top to bottom: increasing upper-limit set in $K$ prior $(\max (C) \times 20, \max (C) \times 100, \max (C) \times 1000)$. 
Table 9: Oblique banded rattail (CAS): Posterior estimates

\begin{tabular}{|c|c|c|c|c|c|c|c|c|c|}
\hline & \multicolumn{3}{|c|}{$\mathrm{E}(r)=0.1 \operatorname{Var}(r)=0.25^{2}$} & \multicolumn{3}{|c|}{$\mathrm{E}(r)=0.4, \operatorname{Var}(r)=0.25^{2}$} & \multicolumn{3}{|c|}{$\mathrm{E}(r)=\mathbf{0 . 8}, \operatorname{Var}(r)=\mathbf{0 . 2 5 ^ { 2 }}$} \\
\hline & mean & median & $\begin{array}{c}95 \% \\
\text { HPD interval }\end{array}$ & mean & median & $\begin{array}{c}95 \% \\
\text { HPD interval }\end{array}$ & mean & median & $\begin{array}{c}95 \% \\
\text { HPD interval }\end{array}$ \\
\hline \multicolumn{10}{|c|}{$K$ prior: upper limit $\max (C) \times 20$} \\
\hline$r$ & 0.21 & 0.14 & $(0.01,0.65)$ & 0.42 & 0.39 & $(0.10,0.80)$ & 0.71 & 0.69 & $(0.39,1.1)$ \\
\hline$K$ & 10 & 7 & $(1,29)$ & 5 & 4 & $(1,12)$ & 3 & 2 & $(1,6)$ \\
\hline$q$ & 0.305 & 0.2269 & $(0.0088,0.8262)$ & 0.4818 & 0.4105 & $(0.0389,1.086)$ & 0.7323 & 0.6694 & $(0.1262,1.473)$ \\
\hline$\sigma_{u}^{2}$ & 0.0097 & 0.0971 & $(0.0003,0.1935)$ & 0.0954 & 0.0933 & $(0.0003,0.185)$ & 0.0917 & 0.0908 & $(0.0002,0.1848)$ \\
\hline$\sigma_{v}^{u}$ & 0.0365 & 0.0077 & $(0.0001,0.1796)$ & 0.0325 & 0.0069 & $(0.0001,0.1606)$ & 0.0358 & 0.0072 & $(0.0001,0.1611)$ \\
\hline MSY & 0.32 & 0.27 & $(0.01,0.78)$ & 0.45 & 0.37 & $(0.13,0.96)$ & 0.48 & 0.41 & $(0.19,0.96)$ \\
\hline \multicolumn{10}{|c|}{$K$ prior: upper limit $\max (C) \times 100$} \\
\hline$r$ & 0.14 & 0.08 & $(0.01,0.49)$ & 0.39 & 0.35 & $(0.09,0.75)$ & 0.71 & 0.69 & $(0.36,1.07)$ \\
\hline$K$ & 30 & 14 & $(1,109)$ & 9 & 5 & $(1,27)$ & 4 & 2 & $(1,7)$ \\
\hline$q$ & 0.1858 & 0.1175 & $(0.0021,0.5871)$ & 0.4024 & 0.3329 & $(0.0081,0.9975)$ & 0.6981 & 0.6359 & $(0.0734,1.478)$ \\
\hline$\sigma_{u}^{2}$ & 0.1029 & 0.1001 & $(0.0002,0.1969)$ & 0.0096 & 0.0944 & $(0.0003,0.1873)$ & 0.0908 & 0.0904 & $(0.0003,0.1839)$ \\
\hline$\sigma_{v}^{2}$ & 0.0339 & 0.0067 & $(0.0001,0.1724)$ & 0.0337 & 0.0065 & $(0.0001,0.1687)$ & 0.0369 & 0.0069 & $(0.0001,0.1698)$ \\
\hline MSY & 0.55 & 0.3 & $(0.01,1.7)$ & 0.64 & 0.41 & $(0.1,1.7)$ & 0.56 & 0.42 & $(0.2,1.15)$ \\
\hline \multicolumn{10}{|c|}{$K$ prior: upper limit $\max (C) \times 1000$} \\
\hline$r$ & 0.11 & 0.06 & $(0.01,0.4)$ & 0.37 & 0.34 & $(0.08,0.74)$ & 0.71 & 0.69 & $(0.37,1.1)$ \\
\hline$K$ & 90 & 23 & $(1,419)$ & 20 & 5 & $(1,81)$ & 4 & 2 & $(1,8)$ \\
\hline$q$ & 0.1394 & 0.0676 & $(0.0008,0.4798)$ & 0.3678 & 0.2919 & $(0.0024,0.9877)$ & 0.6938 & 0.6352 & $(0.0186,1.437)$ \\
\hline$\sigma_{u}^{2}$ & 0.1025 & 0.0097 & $(0.0003,0.198)$ & 0.0942 & 0.0929 & $(0.0003,0.1851)$ & 0.0908 & 0.0907 & $(0.0002,0.1836)$ \\
\hline$\sigma_{v}^{u}$ & 0.0363 & 0.0073 & $(0.0002,0.1807)$ & 0.0383 & 0.0074 & $(0.0001,0.1815)$ & 0.0381 & 0.0069 & $(0.0002,0.1754)$ \\
\hline MSY & 1.5 & 0.38 & $(0.01,5.9)$ & 1.4 & 0.4 & $(0.08,4.8)$ & 0.6 & 0.42 & $(0.19,1.3)$ \\
\hline
\end{tabular}



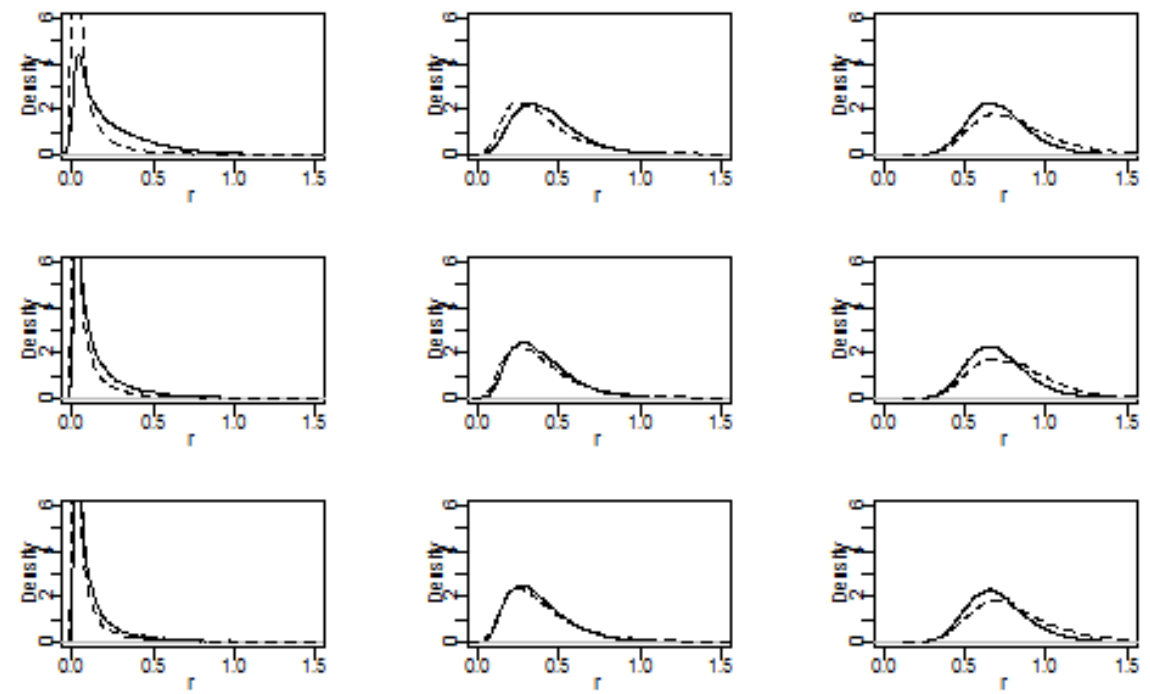

Figure 56: Oblique banded rattail (CAS): prior and posterior distributions for $r$. Solid line is posterior distribution, dashed line is prior distribution. Left to right: increasing value of $\mathrm{E}(r)=(0.1,0.4,0.8)$ specified in $r$ prior. Top to bottom: increasing upper-limit set in $K$ prior $(\max (C) \times 20, \max (C) \times 100, \max (C) \times 1000)$.
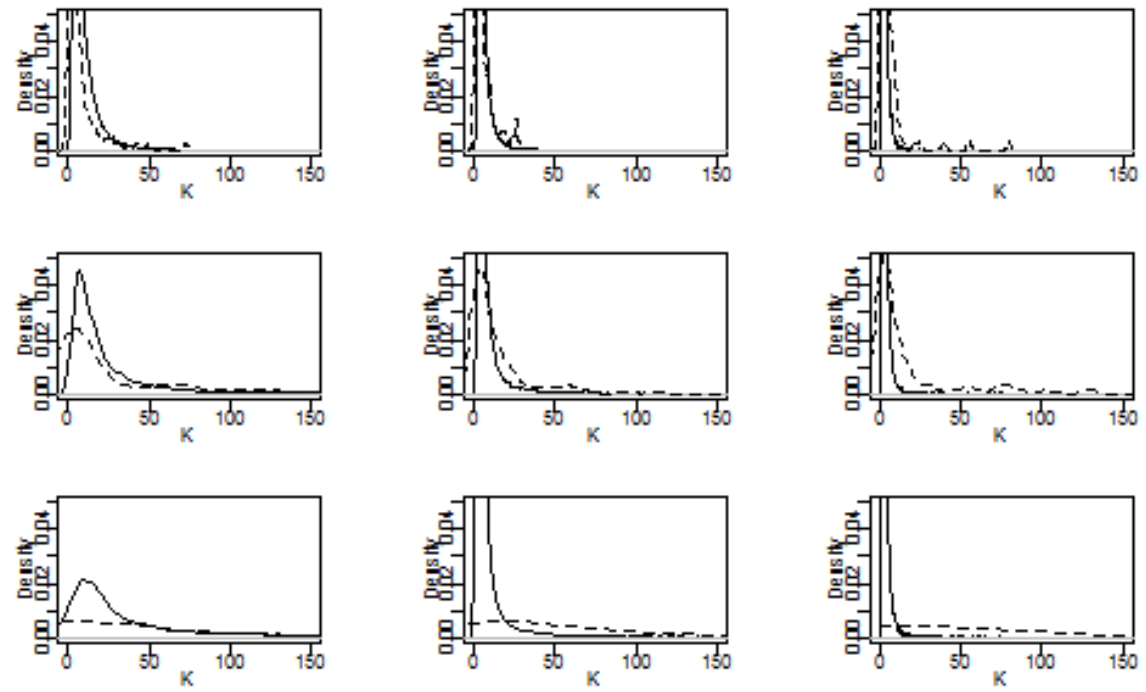

Figure 57: Oblique banded rattail (CAS): prior and posterior distributions for $K$. Solid line is posterior distribution, dashed line is prior distribution. Left to right: increasing value of $\mathrm{E}(r)=(0.1,0.4,0.8)$ specified in $r$ prior. Top to bottom: increasing upper-limit set in $K$ prior $(\max (C) \times 20, \max (C) \times 100, \max (C) \times 1000)$. 


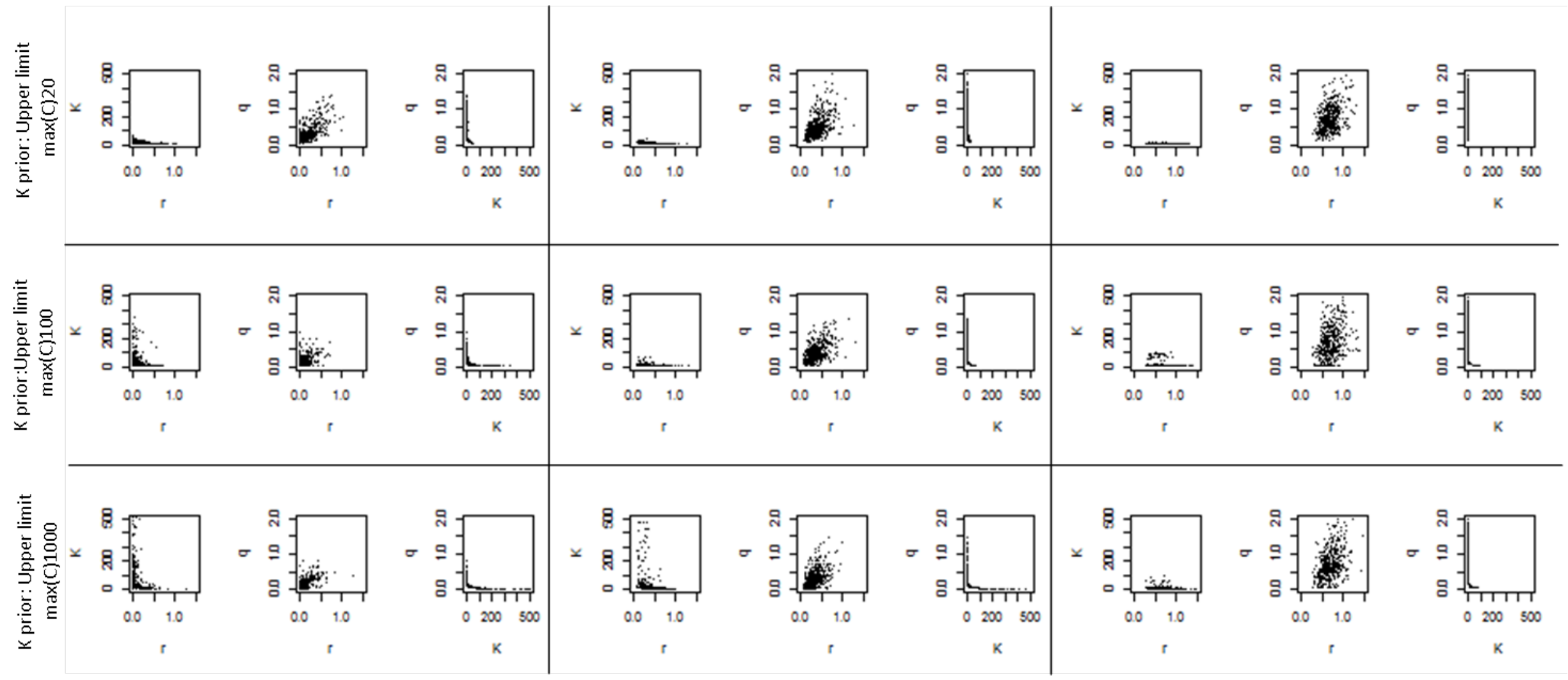

Figure 58: CAS: parameter correlations $\mathrm{r}, \mathrm{K}, \mathrm{q}$ 

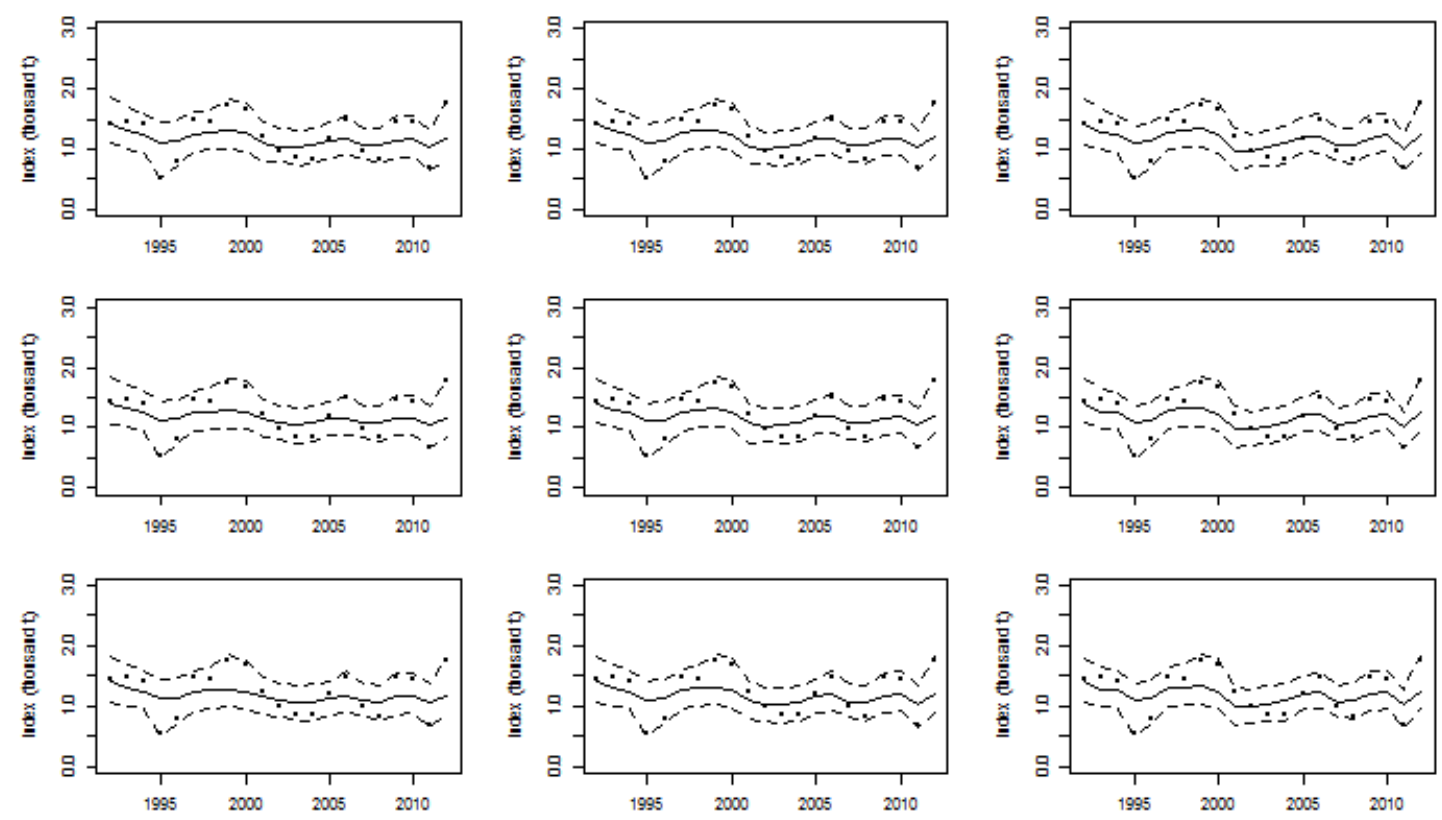

Figure 59: Oblique banded rattail (CAS): Plot of observed index $\left(I_{t}=\bullet\right)$ and posterior median for the model predicted index (solid line) with its 95\%HPD interval (dashed line).Left to right: increasing value of $\mathrm{E}(r)=(0.1,0.4,0.8)$ specified in $r$ prior. Top to bottom: increasing upper-limit set in $K$ prior $(\max (C) \times 20, \max (C) \times 100, \max (C) \times 1000)$.
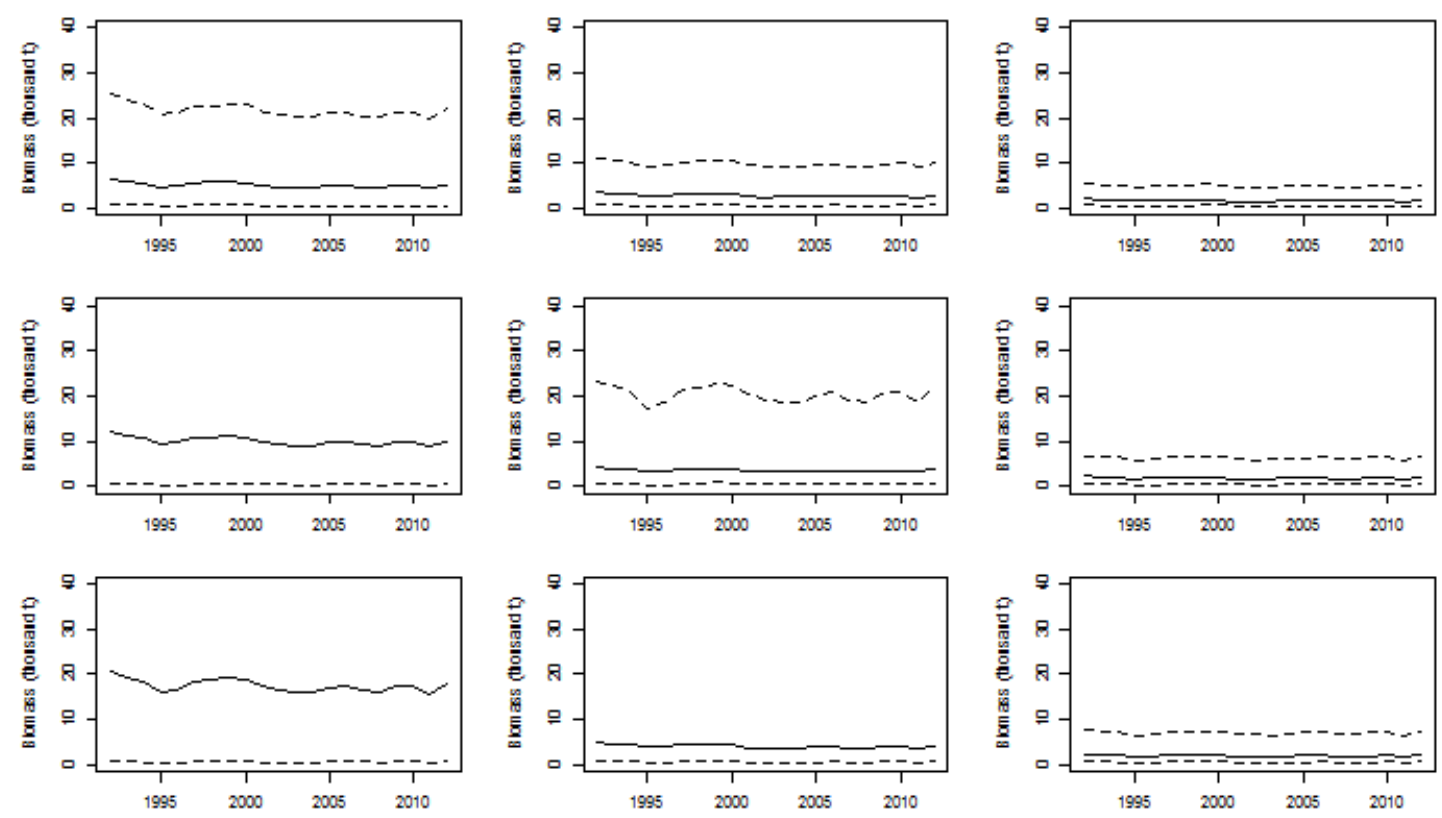

Figure 60: Oblique banded rattail (CAS): Plot of the posterior median for the model predicted index (solid line) with its 95\%HPD interval (dashed line).Left to right: increasing value of $\mathrm{E}(r)=(0.1,0.4,0.8)$ specified in $r$ prior. Top to bottom: increasing upper-limit set in $K$ prior $(\max (C) \times 20, \max (C) \times 100, \max (C) \times 1000)$. 